\title{
The Atmospheric Dynamics of Venus
}

\author{
Agustín Sánchez-Lavega ${ }^{(1)}$ \\ Universidad del Pais Vasco UPV/EHU \\ Sebastien Lebonnois ${ }^{(2)}$ \\ Laboratoire de Meteorologie Dynamique
}

Takeshi Imamura ${ }^{(3)}$

The University of Tokio

${ }^{1}$ Departamento de Física Aplicada I, Escuela de Ingeniería de Bilbao, Universidad del País Vasco UPV/EHU, Alameda Urquijo s/n, 48013 Bilbao. Spain.

${ }^{2}$ Laboratoire de Météorologie Dynamique, Jussieu, Box 99, 75252 Paris 05, France

${ }^{3}$ Department of Complexity Science and Engineering, Graduate School of Frontier Sciences, The University of Tokyo, 5-1-5 Kashiwanoha, Kashiwa, Chiba 277-8561, Japan

${ }^{4}$ Atmospheric, Oceanic \& Planetary Physics, University of Oxford, Oxford, UK

${ }^{5}$ Instituto de Astrofísica, Observatório Astronómico de Lisboa, Tapada da Ajuda, 1349-018 Lisboa, and Faculdade de Ciências, Universidade de Lisboa, 1749-016 Lisboa, Portugal.

${ }^{6}$ Laboratoire d'Etudes Spatiales et d'Instrumentation en Astrophysique, Observatoire de Paris, section de Meudon, 5 Place Jules Janssen, 92195 Meudon CEDEX, France.

\begin{abstract}
We review our current knowledge of the atmospheric dynamics of Venus prior to the Akatsuki mission, in the altitude range from the surface to approximately the cloud tops located at about $100 \mathrm{~km}$ altitude. The three-dimensional structure of the wind field in this region has been determined with a variety of techniques over a broad range of spatial and temporal scales (from the mesoscale to planetary, from days to years, in daytime and nighttime), spanning a period of about 50 years (from the 1960s to the present). The global panorama is that the mean
\end{abstract}


atmospheric motions are essentially zonal, dominated by the so-called super-rotation (an atmospheric rotation that is 60 to 80 times faster than that of the planetary body). The zonal winds blow westward (in the same direction as the planet rotation) with a nearly constant speed of $\sim 100 \mathrm{~m} \mathrm{~s}^{-1}$ at the cloud tops (65-70 km altitude) from latitude $50^{\circ} \mathrm{N}$ to $50^{\circ} \mathrm{S}$, then decreasing their speeds monotonically from these latitudes toward the poles. Vertically, the zonal winds decrease with decreasing altitude towards velocities $\sim 1-3 \mathrm{~m} \mathrm{~s}^{-1}$ in a layer of thickness $\sim 10 \mathrm{~km}$ close to the surface. Meridional motions with peak speeds of $\sim 15 \mathrm{~m} \mathrm{~s}^{-1}$ occur within the upper cloud at $65 \mathrm{~km}$ altitude and are related to a Hadley cell circulation and to the solar thermal tide. Vertical motions with speeds $\sim 1-3 \mathrm{~m} \mathrm{~s}^{-1}$ occur in the statically unstable layer between altitudes of $\sim 50-55 \mathrm{~km}$. All these motions are permanent with speed variations of the order of $\sim 10 \%$. Various types of wave, from mesoscale gravity waves to Rossby-Kelvin planetary scale waves, have been detected at and above cloud heights, and are considered to be candidates as agents for carrying momentum that drives the super-rotation, although numerical models do not fully reproduce all the observed features. Momentum transport by atmospheric waves and the solar tide is thought to be an indispensable component of the general circulation of the Venus atmosphere. Another conspicuous feature of the atmospheric circulation is the presence of polar vortices. These are present in both hemispheres and are regions of warmer and lower clouds, seen prominently at infrared wavelengths, showing a highly variable morphology and motions. The vortices spin with a period of 2-3 days. The South polar vortex rotates around a geographical point which is itself displaced from the true pole of rotation by $\sim 3$ degrees. The polar vortex is surrounded and constrained by the cold collar, an infrared-dark region of lower temperatures. We still lack detailed models of the mechanisms underlying the dynamics of these features and how they couple (or not) to the super-rotation. The nature of the super-rotation relates to the angular momentum stored in the atmosphere and how it is transported between the tropics and higher latitudes, and between the deep atmosphere and upper levels. The role of eddy processes is crucial, but likely involves the complex interaction of a variety of different types of eddy, either forced directly by radiative heating and mechanical interactions with the surface or through various forms of instability. Numerical models have achieved some significant recent success in capturing some aspects of the observed super-rotation, consistent with the scenario discussed by Gierasch (1975) and Rossow and Williams (1979), but many uncertainties remain, especially in the deep atmosphere. The theoretical framework developed to explain the circulation in Venus's atmosphere is reviewed, as well as the numerical models that have been built to elucidate the super-rotation mechanism. These tools are used to analyze the respective roles of the different waves in the processes driving the observed motions. Their limitations and suggested directions for improvements are discussed.

\section{9}

\section{Introduction}

This chapter reviews our current knowledge of Venus atmospheric dynamics prior to the Japanese JAXA mission, Akatsuki, in the altitude range from the surface to about $100 \mathrm{~km}$, fully encompassing the region of thick clouds. The atmospheric pressure and temperatures decrease with height across this region from 91.2 bar and $735 \mathrm{~K}$ at the surface to 0.03 mbar and $175 \mathrm{~K}$ at $100 \mathrm{~km}$ (not far from the conditions found on Earth at 80-90 km). The approximate mean lapse rate is $\sim 8 \mathrm{~K} \mathrm{~km}^{-1}$, the highest value found in any planetary atmosphere within the solar system. 
$\mathrm{mol}^{-1}$, also the highest value in a Solar System planetary atmosphere), the atmosphere between 0 and $100 \mathrm{~km}$ is massive when compared to those of the other two terrestrial planets and Titan. Importantly for atmospheric dynamics, this layer contains most of the aerosols and cloud particles (about $75 \%$ made of $\mathrm{H}_{2} \mathrm{SO}_{4}$ droplets) in the Venus atmosphere extending vertically in the altitude range from $\sim 30$ to $80 \mathrm{~km}$. The proximity of Venus to the Sun, closer than that of Earth, means that the solar irradiance is 1.9 times as large. About $80 \%$ of the solar radiation is reflected by these clouds. Most solar heat is deposited in and above the clouds with the radiative time constant decreasing rapidly with height from $\sim 116$ days at $40 \mathrm{~km}$ (lower cloud deck) to $0.5 \mathrm{hr}$ at $100 \mathrm{~km}$. Taking into account that the length of a solar day is 117 days, this suggests that the expected solar induced dynamical variability should occur in the upper part of the layer under study. In addition, because the orbital eccentricity and obliquity of the planetary rotation axis are small, no substantial seasonal changes are expected. But the essential ingredient that distinguishes Venus atmospheric dynamics from the others of the solar system is its superrotation state at the upper cloud level. Venus's solid globe has a rotation period of 243 days, but the upper cloud layer accessible to visible wavelengths rotates with a period of $\sim 4$ days; a 60 times shorter than the planet. There is no other equivalent in the solar system, so Venus's atmosphere is a singular and archetypical case for the study of dynamics under slow rotation of the solid body. All these aspects have been integrated in Global Circulation Models (GCMs) that try to simulate and help elucidate the three-dimensional structure of the observed wind field in the lowest $100 \mathrm{~km}$ of the atmosphere. The above numbers summarising these properties are taken from Sanchez-Lavega (2011) and can also be found in the other chapters of this book.

Previous reviews on this subject were written as information arrived from successive space missions, the earliest ones by Moroz (1981) and Schubert (1983) covering the results from the Pioneer-Venus mission and and the Soviet Venera missions up to Venera 14. Gierasch et al. (1997) also covered atmospheric studies resulting from the Soviet Vega balloon exploration missions and from the Galileo flyby. Until the arrival of Venus Express at the planet in 2005, the newly available data on the atmosphere during the period after the Galileo flyby were obtained with ground-based telescopes, since there were no other space missions to study Venus. A recent book entitled 'Towards understanding the Climate of Venus' (2013) that includes Venus Express data (reviewed also by Drossart and Montmessin, 2015), contains many chapters dealing with atmospheric dynamics (Limaye and Rengel, Read, Lewis et al., Lebonnois et al.). A list of spacecraft missions to Venus can be found in the Venus I book with some additions in Venus book II, and is updated in Taylor et al. (this book).

\section{Wind data}

In this section we describe the three-dimensional structure of the wind field and its temporal variability as measured by a plethora of instruments on landers, balloons and orbiting spacecrafts

\subsection{Wind measurement methods}

The most commonly used methods to determine the winds of Venus are described in what follows. In Table 1 we present a summary of the methods and measurements so far performed. 


\subsubsection{Cloud tracking $(0.35-5 \mu m)$.}

136

137

138

139

140

141

142

143

144

145

146

147

148

149

150

151

152

153

154

155

156

157

158

159

160

161

162

163

164

165

166

167

168

169

170

171

172

173

174

175

176

177

178

In this approach, cloud elements are assumed to act as passive tracers of the flow, allowing the measurement of horizontal winds. Caution must be taken in identifying periodic cloud patterns whose motions may be representative of the phase velocity of waves relative to the background flow (see section 4). The following dynamic processes can lead to a misinterpretation of the measured global wind speed: (a) Waves (observed wavelengths $\sim 50$ - 2,000 km), (b) Solar tides (local time dependence), (c) Cloud processes changing the element morphology (expansion, contraction, evaporation or changes in absorber or opacity), (d) Vorticity and rotating features around a vortex (but rare in Venus outside the poles).

In day time, images of the cloud field are observed in reflected sunlight and it is at ultraviolet and violet wavelengths $(350-410 \mathrm{~nm}, \mathrm{UV})$ and in the near infrared $(950-980 \mathrm{~nm})$ where the contrast between cloud elements is higher, due to unknown absorbing chromophore(s), and where cloud features are easy to identified and track. At 350-410 nm we sense clouds at altitudes $\sim 65-70 \mathrm{~km}$ while at $950-980 \mathrm{~nm}$ we sense clouds at $\sim 60 \mathrm{~km}$. Wind profiles retrieved from tracking UV markings have been derived from orbital or fly-by missions, including Mariner 10 (Limaye and Suomi, 1981); Pioneer-Venus (Limaye, 2007); Galileo (Peralta et al., 2007; Kouyama et al., 2012); Venus Express - VIRTIS (Sanchez-Lavega et al., 2008; Hueso et al., 2012, 2015); Venus Express-VMC (Khatuntsev et al., 2013), and also recently from groundbased telescopes (Sánchez-Lavega et al., 2016). On the night side we measure the motions of cloud features identified by their opacity contrast in thermal emission at near infrared wavelengths at 1.7 and $2.2 \mu \mathrm{m}$ (altitude sensed $\sim 45-50 \mathrm{~km}$ ) and as excess emission relative to the background in the polar regions at 3.8 and $5.1 \mu \mathrm{m}$ (altitude sensed $\sim 65 \mathrm{~km}$ ). In addition, imaging in the 8-22 $\mu \mathrm{m}$ region has been used to capture the upper cloud at low contrast, except in the polar areas where the features detach (Taylor et al., 1980; Orton et al., 1991; Sato et al., 2014).

\subsubsection{Entry and landing probes.}

The motion of probes descending through the atmosphere is used to measure the Doppler shifted radio wavelengths they send to an orbiter or the Earth itself as the probe is dragged by the winds (sounded wind altitudes $0-65 \mathrm{~km}$ ). The Pioneer-Venus (PV) probes used Doppler shifted radio signals to determine the velocity component along the Earth-Venus line of sight, while the two other orthogonal components were retrieved using differential very-long-baseline interferometry techniques (Counselman et al., 1980). The probe data refer to the particular latitude-longitude of the descent and its local time. There are vertical wind profiles available for the Venera (V) missions V4, V7, V8, V9-V10, V12 (Kerzhanovich and Marov, Venus I, p. 766), V13 (Avduevskiy et al., Venus I, p. 280, Ch. 12), Vega 1 and 2 (Moroz and Zasova, 1997) and for the Pioneer-Venus Day, Night, North and Sounder multi-probes (Coulseman et al., 1980). Direct measurements of surface winds were determined by anemometers on the probes Venera 9 and 10 or using acoustic sensors on Venera 13 and 14 (Moroz in Venus I, p.58). These are the only methods so far used to measure winds at heights $\leq 45 \mathrm{~km}$. Caution must be taken, however, concerning the measured wind direction by the Venera probes (Kherzanovich and Marov, 1983, fig. 1). A map of the entry locations of the probes (latitude-longitude and local time) can be found in Schubert (1983, figure 1) and Kherzanovich and Marov (1983, figure 8). 


\subsubsection{Balloons.}

Two balloons were deposited in 1985 in the Venus atmosphere at an altitude of $\sim 53 \mathrm{~km}$ by the Vega 1 and Vega 2 spacecraft. They were used to measure winds by Doppler-shifted radio signals from global tracking, and by means of onboard anemometers for vertical winds in this layer (Blamont et al., 1986; Linkin et al., 1986). No other similar experiment has so far been performed.

\subsubsection{Doppler spectroscopy techniques.}

The line-of-sight wind velocity is determined from Doppler-shifted lines from ground-based observations of three types of spectra: (a) Visible sunlight radiation reflected at the upper clouds (Fraunhofer solar spectrum) and atmospheric $\mathrm{CO}_{2}$ absorption (altitudes sounded 65-75 km). This technique allows also to measure the meridional wind component (Machado et al., 2017). The The Doppler and cloud tracking techniques have repeatedly shown agreement on measurements of wind velocities (Machado et al. 2014; 2017).; (b) CO absorption lines at millimeter and submillimeter wavelengths (altitudes sounded 90-105 km); (c) $\mathrm{CO}_{2}$ non-LTE emissions at 5 and $10 \mu \mathrm{m}$ (altitudes sounded $110 \mathrm{~km}$ ). See Lellouch et al. (1997) and Limaye (2013) for a

\subsubsection{Tracking emission features from $\mathrm{O}_{2}$ (airglow) and $\mathrm{NO}$.}

Distinct emission elements (as bright spots and filaments) are observed at $1.27 \mu \mathrm{m}$ (from $\mathrm{O}_{2}$ ) and in the ultraviolet at 190-300 nm (from NO) and their tracking is used to retrieve motions in the high atmosphere (altitudes 95-110 km) (see Drossart and Montmessin, 2015 and references therein; Gerard et al., this book). However, it is questionable whether these features can be considered as passive tracers of the atmospheric flow.

\subsubsection{Cyclostrophic balance.}

This is an indirect method used for wind determination. Radio-occultation profiles and thermal radiation spectra are used to retrieve the three-dimensional pressure and temperature field (altitudes 45 - 90 km) (Newman et al., 1984; Limaye, 1985; Piccialli et al., 2008). Assuming that the atmosphere is in cyclostrophic balance, the winds can be retrieved from meridional pressure gradients or temperature gradients (Sanchez-Lavega, 2011; see sections 2.5 and 3 for details).

\subsection{Zonal winds: The Super-rotation}

In this section we present all the available data on the three-dimensional structure of the zonal winds that dominate Venus's atmospheric circulation and confirm what is called the Retrograde Super-rotation (RSR). As a convention, we define the rotation of the atmosphere and measure wind speeds relative to the solid body of Venus that rotates in a direction contrary relative to the Earth's rotation or to the general momentum direction of the bodies in the solar system. Then Venus rotation is retrograde. The zonal winds are therefore negative and accordingly are said 
retrograde (as indicated above, RSR). However, from the atmospheric dynamics point of view it must be understood that the zonal winds are "prograde" relative to the surface, i. e. they flow in the same sense as the surface rotation.

We focus in this paper on the measurements in the altitude range from the surface to $\sim 70 \mathrm{~km}$. In section 2.5 we briefly review some data for the $70-100 \mathrm{~km}$ range since this topic is addressed in Chapter IX.

\subsubsection{Zonal wind: Vertical structure.}

The direct measurements of winds by the methods above shows unambiguously that, independent of the latitude and local time, the vertical profile of Venus's mean winds are (Schubert, 1983; Gierasch et al., 1997; see Figure 1): (a) predominantly zonal between $z \sim 10$ $100 \mathrm{~km}$ with the flow directed from East to West (westward); (b) these zonal winds increase in speed with altitude from $z \sim 10$ to $65-70 \mathrm{~km}$, being weak $\left(u<2 \mathrm{~m} \mathrm{~s}^{-1}\right)$ below $10 \mathrm{~km}$; (c) the zonal winds reach peak velocities $u \sim 100 \mathrm{~m} \mathrm{~s}^{-1}$ at $\sim 65 \mathrm{~km}$ in the upper cloud deck, implying that the cloud elements have a rotation period around $\sim 4$ days. Since the planet's sidereal rotation period is 243 days, the ratio of the upper cloud rotation period to that of the planet is $\sim 60$ and the atmosphere is said to be in a state of "super-rotation"; (d) the vertical profile of the meridional component of the wind shows fluctuations in this altitude range $(z \sim 0-60 \mathrm{~km})$ but they always have $v< \pm 10 \mathrm{~m} \mathrm{~s}^{-1}$ (+ northward, - southward). All methods employed in the wind measurements give consistent results in the vertical profiles (within the observed fluctuations with latitude and local time). For example, those determined by the probes agree with those measured by the Vega 1 and 2 balloons at $z=53 \mathrm{~km}$ and with those from cloud tracking at $z$ 45-50 km (nighttime) and at $\mathrm{z} \sim 60$ and 65-70 km (daytime). Peralta et al. (2017) show the altitude sensitivity in Venus atmosphere as a function of wavelength (day and night time). Since the measurements cover a period of about 50 years, a firm conclusion about Venus's superrotation and wind field below the upper clouds is that it is permanent in time, with fluctuations in velocity smaller than $10-20 \%$. Good examples of this are shown by the following comparisons between precise in-situ probes and balloon results (i)-(iii):

Table 1 Wind measurement methods $(\mathrm{z}=0-100 \mathrm{~km})$

\begin{tabular}{|l|l|l|l|l|}
\hline Technique & Mission(s) & Trajectory & Epoch & Notes \\
\hline $\begin{array}{l}\text { Doppler } \\
\text { spectroscopy }\end{array}$ & $\begin{array}{l}\text { Ground-based } \\
\text { Optical and } \\
\text { Radio }\end{array}$ & $\begin{array}{l}\text { Remote } \\
\text { sensing }\end{array}$ & 1987 - present & $\begin{array}{l}\text { Fraunhofer spectra } \\
(\mathrm{z}=65-75 \mathrm{~km}) \\
\text { Radio }(\mathrm{mm}-\mathrm{submm}) \\
(\mathrm{z}=90-105 \mathrm{~km})\end{array}$ \\
\hline $\begin{array}{l}\text { Tracking } \mathrm{O}_{2}, \\
\text { NO emissions }\end{array}$ & $\begin{array}{l}\text { Ground-based } \\
\text { Venus Express }\end{array}$ & $\begin{array}{l}\text { Remote } \\
\text { sensing } \\
\text { Orbit }\end{array}$ & 1979 - present & $\begin{array}{l}\text { Airglow } \mathrm{O}_{2} \text { and NO } \\
\text { emissions } \\
(\mathrm{z}=95-110 \mathrm{~km})\end{array}$ \\
\hline $\begin{array}{l}\text { Radio } \\
\text { Doppler } \\
\text { tracking }\end{array}$ & $\begin{array}{l}\text { Venera } \\
4,7,8,9,10, \\
12,13\end{array}$ & $\begin{array}{l}\text { Entry-probe } \\
\text { (descent) }\end{array}$ & $\begin{array}{l}\text { 29 Aug. 1967; 10 } \\
\text { Nov. 1970; 22Jul. } \\
\text { 1972; 22 Oct. 1975; } \\
\text { 25 Oct. 1975; 21 Dec. }\end{array}$ & $\begin{array}{l}\text { Vertical wind profile } \\
(\mathrm{z}=0 \text { - 60 km) }\end{array}$ \\
\hline
\end{tabular}




\begin{tabular}{|c|c|c|c|c|}
\hline & & & $\begin{array}{l}1978 ; \\
30 \text { Oct. 1981; } 4 \text { Nov. } \\
1981\end{array}$ & \\
\hline $\begin{array}{l}\text { Radio } \\
\text { Doppler } \\
\text { tracking }\end{array}$ & $\begin{array}{l}\text { Pioneer Venus: } \\
\text { Sounder, } \\
\text { North, Day, } \\
\text { Night } \\
\end{array}$ & Entry-probes & 9 Dec. 1978 & $\begin{array}{l}\text { Vertical wind profile } \\
(\mathrm{z}=0-60 \mathrm{~km})\end{array}$ \\
\hline $\begin{array}{l}\text { Radio } \\
\text { Doppler } \\
\text { tracking }\end{array}$ & Vega 1,2 & $\begin{array}{l}\text { Entry-probes } \\
\text { (descent) }\end{array}$ & 11 and 15 June 1984 & $\begin{array}{l}\text { Vertical wind profile } \\
(\mathrm{z}=0-60 \mathrm{~km})\end{array}$ \\
\hline $\begin{array}{l}\text { Anemometer } \\
\text { Acoustic } \\
\text { sensors }\end{array}$ & \begin{tabular}{|l} 
Venera $9-10$ \\
Venera $13-14$
\end{tabular} & $\begin{array}{l}\text { Landing } \\
\text { Landing }\end{array}$ & $\begin{array}{l}22-25 \text { Oct } 1975 \\
30 \text { Oct-4 Nov } 1981\end{array}$ & $\begin{array}{l}\text { Surface winds } \\
\text { Surface winds }\end{array}$ \\
\hline \begin{tabular}{|l} 
Cloud \\
tracking in \\
images \\
\end{tabular} & $\begin{array}{l}\text { Mariner } 10 \\
\text { NASA }\end{array}$ & Flyby & 5 Feb 1974 & $\begin{array}{l}\text { Vidicon cameras (UV) } \\
(\mathrm{z}=65-70 \mathrm{~km})\end{array}$ \\
\hline $\begin{array}{l}\text { Cloud } \\
\text { tracking in } \\
\text { images }\end{array}$ & Pioneer Venus & Orbit & $\begin{array}{l}1979-1985 \\
5 \text { periods of } 80 \text { days }\end{array}$ & $\begin{array}{l}\text { OCPP: UV }(365 \mathrm{~nm}) \\
\text { Winds at } \mathrm{z}=65-70 \\
\mathrm{~km} . \\
\text { Latitude coverage } \sim \\
80^{\circ} \mathrm{S}-70^{\circ} \mathrm{N} \\
\end{array}$ \\
\hline Balloons & Vega 1 and 2 & $\begin{array}{l}\text { Orbiter - } \\
\text { balloons }\end{array}$ & 11-15 Jun. 1985 & Winds at $\mathrm{z}=53 \mathrm{~km}$ \\
\hline $\begin{array}{l}\text { Cloud } \\
\text { tracking in } \\
\text { images }\end{array}$ & Galileo & Flyby & Feb. 1990 & $\begin{array}{l}\text { Vio }(400 \mathrm{~nm}), \text { NIR } \\
(980 \mathrm{~nm}), \mathrm{IR}(2.3 \mu \mathrm{m}) \\
\text { Winds at } \mathrm{z}=45-70 \\
\mathrm{~km})\end{array}$ \\
\hline $\begin{array}{l}\text { Cloud } \\
\text { tracking in } \\
\text { images }\end{array}$ & Venus Express & Polar orbit & $2006-2014$ & $\begin{array}{l}\text { Southern Hemisphere } \\
\text { VMC : UV (365 nm); } \\
\text { NIR (965 nm); VIRTIS } \\
\text { M : UV ( } 380 \mathrm{~nm}), \text { NIR } \\
(980 \mathrm{~nm}), \text { IR }(1.7 \mu \mathrm{m}) \\
\text { (Winds at z }=45-70 \\
\text { km) }\end{array}$ \\
\hline $\begin{array}{l}\text { Cloud } \\
\text { tracking in } \\
\text { images }\end{array}$ & $\begin{array}{l}\text { Ground-based } \\
\text { telescopes }\end{array}$ & - & $\begin{array}{l}\sim 1960 \text { - present } \\
1990 \text { - present }\end{array}$ & $\begin{array}{l}\text { UV }(380-400 \mathrm{~nm}) \\
\text { IR }(2.3 \mu \mathrm{m})\end{array}$ \\
\hline $\begin{array}{l}\text { Cyclostrophic } \\
\text { thermal wind } \\
\text { equation }\end{array}$ & $\begin{array}{l}\text { Pioneer-Venus } \\
\text { Venera } 15 \\
\text { Galileo } \\
\text { Venus Express } \\
\end{array}$ & $\begin{array}{l}\text { Orbit and PV } \\
\text { probes }\end{array}$ & $1979-2014$ & $\begin{array}{l}\text { Zonal winds } \\
(\mathrm{z}=40-90 \mathrm{~km})\end{array}$ \\
\hline
\end{tabular}

(i) Day-night profiles: PV night and PV day probes entered on the same day (Dec. 9, 1978) at 260 about the same latitude (-28. $7^{\circ}$ and $-31.7^{\circ}$ respectively) but at different local times (0:07 -night- 
and 06:46 -day-, respectively) (Colin, 1983). The retrieved vertical profiles $u(z)$ are similar in their structure within measurement errors (Coulseman et al., 1980). They also agree with the long-term cloud tracking at three altitudes (47, 60 and $65 \mathrm{~km}$ ) using VIRTIS onboard Venus Express (Sanchez-Lavega et al., 2008; Hueso et al., 2015).

(ii) Day-night winds at points close in latitude and altitude: Vega 1 and 2 balloons were deposited at close equatorial latitudes $\left(+7.3^{\circ}\right.$ and $\left.-6.6^{\circ}\right)$ and at the same altitude $(\mathrm{z}=53 \mathrm{~km})$ and both measured the same velocity $\left(-69 \pm 1\right.$ and $\left.-66 \pm 1 \mathrm{~ms}^{-1}\right)$ in their path of $\sim 11,000 \mathrm{~km}\left(\sim 100^{\circ}\right.$ in longitude) when traveling in both day and night hemispheres (Sagdeev et al., 1986).

(iii) Long-term variability: The PV sounder entered near the equator (latitude $+4.4^{\circ}$ ) on 9 December 1978, at a latitude close to that sounded by the Vega 1 and 2 balloons on 11 and 15 June 1985 (6.5 years later). When comparing the balloons' zonal wind with that from the PV sounder at $z=53 \mathrm{~km}$ we see essentially no difference. They also agree with the winds derived from long-term cloud tracking (2006-2014) at three altitudes (47, 60 and $65 \mathrm{~km}$ ) in equatorial latitudes using VIRTIS onboard Venus Express (Sanchez-Lavega et al., 2008; Hueso et al., 2015).

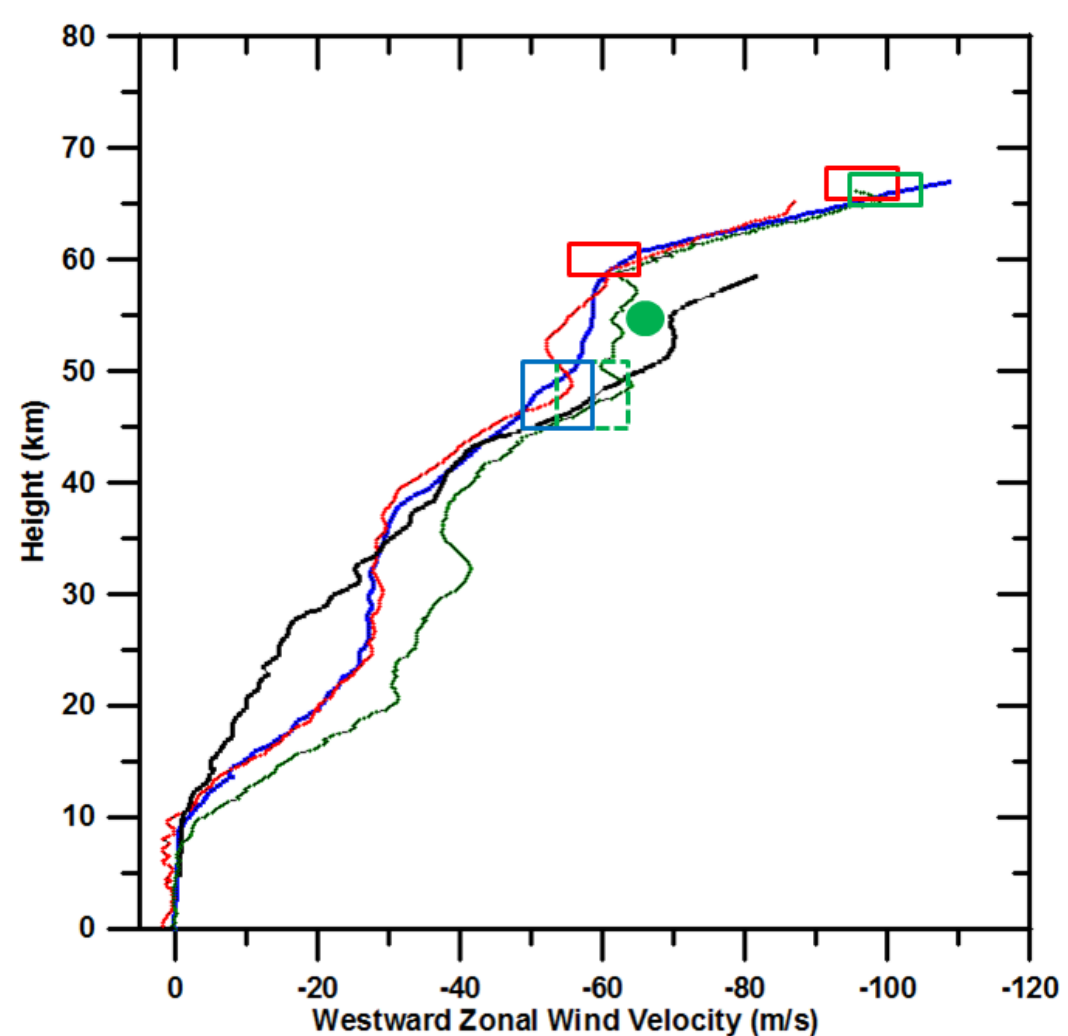

Westward Zonal Wind Velocity $(\mathrm{m} / \mathrm{s})$

Figure 1. Vertical profiles of the zonal winds: comparing different measurements by different methods. Continuous lines as measured by the Pioneer-Venus probes (9 Dec., 1978): Sounder 
(green), North (black), Night (red), Day (blue). The Night and Day probes entered at similar latitudes $\left(-31.7^{\circ},-28.7^{\circ}\right)$ but at different local times $(6: 46,0: 07)$. The green dot is the velocity measured by the Vega 1 and 2 balloons (11-15 June, 1985) at latitudes $\left(+7.3^{\circ},-6.6^{\circ}\right)$ and sounded day and night time. Their data can be compared with the Sounder (or large probe) that entered at latitude $(+4.4 \%$ ). The rectangles are mean values from long-term measurements of cloud tracers using the VIRTIS instrument on Venus Express (2006-2012) at different altitudes: daytime (red) and nighttime (blue).

\subsubsection{Surface winds.}

Direct measurements (anemometers and acoustic sensors) by Venera 8-9, 13-14 gave very low values $\left(0.25-1 \mathrm{~m} \mathrm{~s}^{-1}\right)$ consistent with Doppler measurements by the Venera and PV probes. All probe data (Veneras and PV probes) coincide in showing that the speed from the surface to $\sim 10$ $\mathrm{km}$ altitude is practically constant (independent of solar time and location on the globe) and remains low ( $<2-3 \mathrm{~m} \mathrm{~s}^{-1}$ within measurement errors; Counselman et al., 1980, Kherzanovich and Marov, 1983; Kherzanovich and Limaye, 1985). The Venera 8 wind speed profile from the surface to $11 \mathrm{~km}$ indicates that the wind speed slightly increases with altitude from 0.5 to $3 \mathrm{~m} \mathrm{~s}^{-1}$ (Kerzhanovich and Marov, 1983). This layer with thickness $\sim 10 \mathrm{~km}$ (the scale height at the surface is $\sim 16 \mathrm{~km}$ and $14 \mathrm{~km}$ at $10 \mathrm{~km}$ altitude) contains about $40 \%$ of the atmospheric mass (the atmospheric density is $\sim 65 \mathrm{~kg} \mathrm{~m}^{-3}$ at the surface, decreasing to $\sim 38 \mathrm{~kg} \mathrm{~m}^{-3}$ at $10 \mathrm{~km}$ ), but the mass flow and kinetic energy density of this layer are the lowest anywhere in the atmosphere. The above data refer to measurements at the surface without considering its elevation due to topography relative to the Venus reference geoid. The PV Night probe and the V8 winds were prograde in the $z=0-10 \mathrm{~km}$ altitude range. Lorenz (1996) presented a compilation of wind speeds in the lowest $100 \mathrm{~m}$ of the atmosphere as measured by the Veneras and PV probes, showing in all cases that the wind speed in this layer was $<1 \mathrm{~m} \mathrm{~s}^{-1}$. Kherzanovich and Marov (1983), Dobrovolskis (1993) and Gierasch et al. (1997) discuss the properties and effects of a boundary layer on Venus's atmospheric dynamics and on the excitation of waves, but the basic balances determining their characteristics and vertical extent are at present undetermined. Indirect evidence of surface winds (orientation and strength) was reported by Greeley et al. (1995) who investigated aeolian surface features and found evidence of equatorward winds at high latitudes.

\subsubsection{Zonal wind: meridional profiles}

Horizontal maps of the wind velocity vectors (each vector at a given longitude-latitude position) are obtained from the motions of cloud elements and are used to retrieve the zonal $(u)$ and meridional $(v)$ components on the wind. The East-West (zonal) velocity distribution of the zonal wind velocity in latitude bands gives information on the solar local time dependence. The NorthSouth (meridional) profile of the mean zonal wind gives information on the global pole to pole planetary circulation. As shown in section 2, cloud tracking at different wavelengths (UV, NIR and 1.7 and $2.2 \mu \mathrm{m}$ ) sense different altitude levels, and the above-mentioned East-West and North-South profiles can be applied at the three altitudes, $65-70 \mathrm{~km}$ and $60 \mathrm{~km}$ in daytime and 47 
$330 \mathrm{~km}$ in night time. To complement this, the meridional profile of the zonal wind for the cloud tops $331 \quad(65-70 \mathrm{~km})$ can be compared with that derived from Doppler shifted lines in the reflected solar 332 spectra.

333 Figure 2 shows the meridional profiles of the zonal winds as measured from cloud tracking in the 334 UV in the upper cloud (65-70 km, Venus daytime) along different epochs using a variety of 335 instruments on board different spacecraft missions: Mariner 10 (Limaye and Suomi, 1981); 336 Pioneer-Venus (Rossow et al., 1990; Limaye, 2007); Galileo (Belton et al., 1991; Peralta et al., 337 2007; Kouyama et al., 2012); Venus Express - VIRTIS (Sanchez-Lavega et al., 2008; Hueso et 338 al., 2012, 2015); Venus Express-VMC (Khatuntsev et al., 2013) and ground-based small 339 telescopes (Sánchez-Lavega et al., 2016). The spacecraft temporal coverage extends from 1974 340 to 2014 and two main conclusions are immediately apparent: (a) the profile consists of two parts, 341 a zonal velocity that is nearly constant at $\sim-100 \mathrm{~m} \mathrm{~s}^{-1}$ between latitudes $\sim \pm 45^{\circ}$, decreasing 342 toward the pole with a meridional wind shear $\partial \mathrm{u} / \partial \mathrm{y} \sim 2.5 \times 10^{-5} \mathrm{~s}^{-1}$ (two orders of magnitude 343 higher than the planetary vorticity $f=2 \Omega \sin \varphi=4.7 \times 10^{-7} \mathrm{~s}^{-1}$ ); (b) this profile is quasi-stable in 344 the long-term (so far explored $\sim 40$ years or $\sim 62$ Venus years) with zonal velocity fluctuations $\leq$ $34510 \%$, although an apparent systematic increase in the upper cloud super-rotation has been 346 reported since 2006 (see details in section 2.4.3). It should be noted that the instantaneous 347 velocity fluctuations measured with cloud tracking can be larger than this $10 \%$ from the mean, 348 they involve eddy and wave motions and the cloud top altitude where the winds are measured 349 varies with latitude, decreasing in particular near the poles (Ignatiev et al., 2009; Hueso et al., 350 2015). This result is confirmed by the measurements of the radial and derived zonal velocity 351 profiles obtained from high spatial resolution, Doppler-shifted, solar spectra reflected from 352 Venus’s cloud tops (Widemann et al., 2007, 2008; Machado et al., 2012, 2014).

353 On the night side, the local probe data tend to support the maintenance of the super-rotation at 354 the 65-70 km altitude level (Figure 1) but full wind measurement coverage is required to explore 355 the potential effects of the solar tide, waves and other processes related to the heating/cooling 356 rate daily variability. 


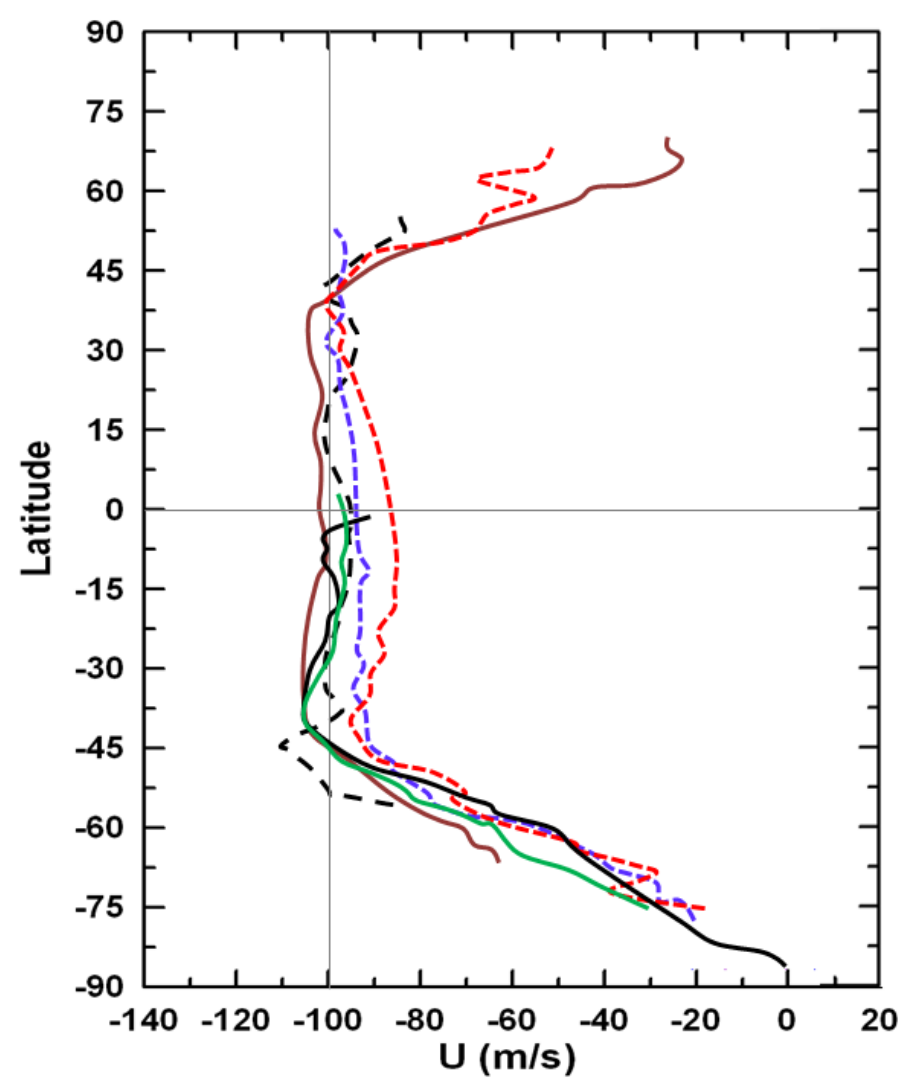

361 Figure 2. Meridional profiles of the zonal wind measured from cloud tracking at UV wavelengths (altitude range 65-70 km). Averages from the following missions: Mariner 10, 1974 (black dashed); Pioneer-Venus,1980 (blue dotted); Pioneer-Venus,1982 (red dotted); Galileo, 1990 (brown line); Venus Express VIRTIS, 2006-2012 (black line); Venus Express VMC, 20063652012 (green line).

366
Figure 3 (left) shows the meridional profile of the zonal wind measured in the 960-980 nm wavelength range at the base of the upper cloud ( $\sim 60 \mathrm{~km}$ height). There are fewer data available than in the UV, and these come from the Galileo mission for the northern hemisphere (Peralta et al, 2007; Kouyama et al., 2012) and from Venus Express for the southern hemisphere with two instruments VIRTIS (Sanchez-Lavega et al., 2008; Hueso et al., 2014) and VMC (Khantuntsev et al., 2013). The measurements show the same trend in the meridional structure of the zonal velocity as in the UV but with decreasing values. The mean constant velocity found between latitudes $\sim \pm 45^{\circ}$ is $70 \mathrm{~ms}^{-1}$, these values being consistent with probe measurements at a lower altitude. 

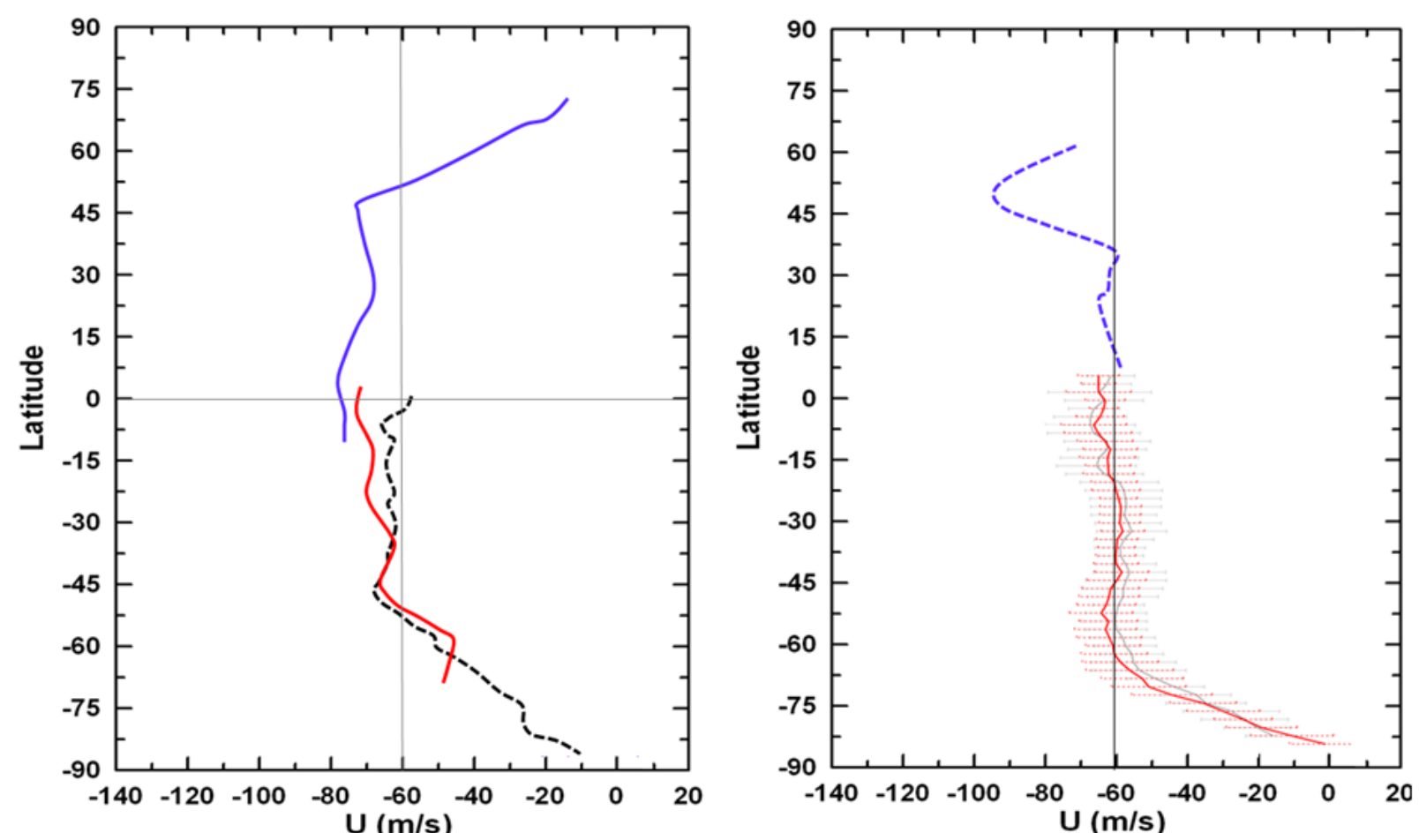

Figure 3. Left: Meridional profiles of the zonal wind measured from cloud tracking at a wavelength of $980 \mathrm{~nm}$ (altitude range $\sim 60 \mathrm{~km}$ ). Averages from the following missions: Galileo, 1990 (blue); Venus Express VIRTIS , 2006-12 (black dotted) VEX; Venus Express VMC , 200612 (red line). Right: Meridional profile of the zonal wind measured from cloud tracking at a wavelength of $1.7 \mu \mathrm{m}$ (altitude range $\sim 45-50 \mathrm{~km}$; nighttime). Averages from the following mission: Galileo, 1990 (blue dashed); Venus Express VIRTIS, 2006-12 (red and thin line, two sets of data) with errors bars indicated.

Finally in Figure 3 (right), we also show the meridional profile of the zonal wind as measured in the night-time in the $1.7 \mu \mathrm{m}$ spectral window that senses motions in the lower cloud at an altitude $\sim 47 \mathrm{~km}$ (Barstow et al., 2010). The data are noisy for the northern hemisphere (Carlson et al, 1991) but relatively good for the southern hemisphere, which come from the Venus Express VIRTIS instrument (Sanchez-Lavega et al., 2008; Hueso et al., 2012). According to the southern hemisphere data, the profile shows a similar pattern to that of the upper cloud, but the latitude where the inflexion point initiates is located poleward, around latitude $-75^{\circ}$.

A firm conclusion from these data is that the zonal winds decrease globally with decreasing altitude within the cloud layers. Extrapolating to the whole planet from the probe measurements of the vertical structure of the zonal winds at particular latitudes and longitudes (and time), a major conclusion is that the zonal winds increase globally in Venus's atmosphere from the surface to around the $65-70 \mathrm{~km}$ altitude level. 


\subsection{Meridional and vertical components of the wind}

This section is devoted to the available measurements of the meridional (North-South) and

\subsubsection{Meridional (North-South) winds.}

The vertical profile of the meridional wind velocity $v(z)$ (North-South - Equatorward) was measured by the North-Day-Night-Sounder PV probes (Counselman et al., 1980, Figure 4). These profiles show that, below the clouds, the meridional winds are very weak $v<1-2 \mathrm{~m} \mathrm{~s}^{-1}$, but in the cloudy region (above $\sim 45 \mathrm{~km}$ ) they vary between -5 and $+5 \mathrm{~ms}^{-1}$. The North-South wind component has been measured on a global scale at the three cloud level altitudes sounded in both daytime (UV and $950 \mathrm{~nm}$ ) and nighttime $(1.74 \mu \mathrm{m})$. This is shown in Figure 5. In the UV at $65-70 \mathrm{~km}$ altitude, the wind data from Pioneer Venus, Galileo and Venus Express (VIRTIS and VMC) show unambiguously that the winds increase from equator to pole, from 0 to $\sim 10-15$ $\mathrm{m} \mathrm{s}^{-1}$ with a peak at latitude $\sim 60^{\circ}$, directed southward in the southern hemisphere $(v<0)$ and northward in the northern hemisphere $(v>0)$. Ground-based measurements of the meridional profile from Doppler-shifted spectral lines confirm this result (Machado et al., 2014, 2017). This structure of the meridional wind has usually been interpreted as a manifestation of the upper branch of a Hadley cell. This meridional motion, however, is not observed in the lower clouds (60 and $45 \mathrm{~km}$ ) (Figure 5), as might have been expected from the PV probes data. It remains open, therefore, to know if the variations seen in the probe data for $v(z)$ are due to the lower branch or branches of the Hadley cell, as interpreted by Schubert (1983), or are due to vertically propagating gravity waves (Gierasch et al., 1997).

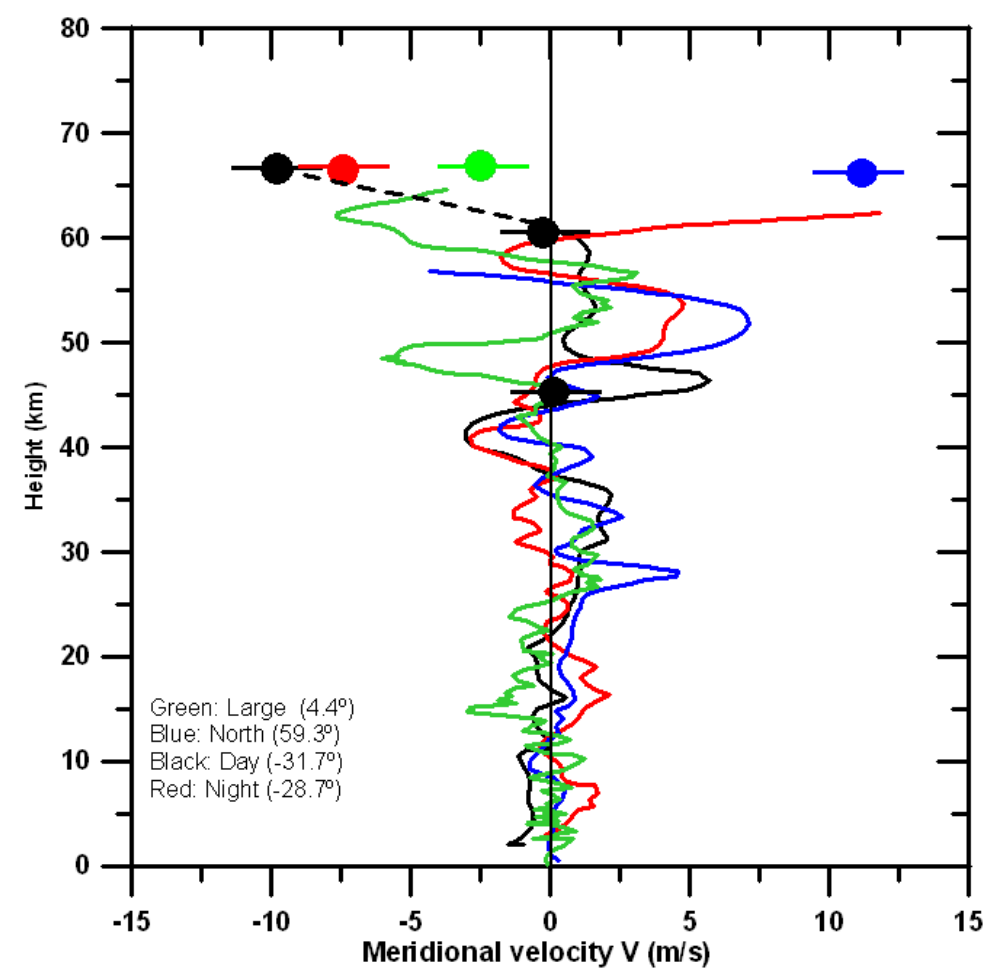


435
Figure 4. Vertical profile of the meridional (North-South) velocity as measured by the PioneerVenus probes as indicated. For comparison, the points show the averaged meridional winds determined at the same latitude and approximately the same altitude as measured from cloud tracking as shown in Figures 2-3. The dashed line indicates the vertical tendency of the meridional winds from cloud tracking measurements (black dots).

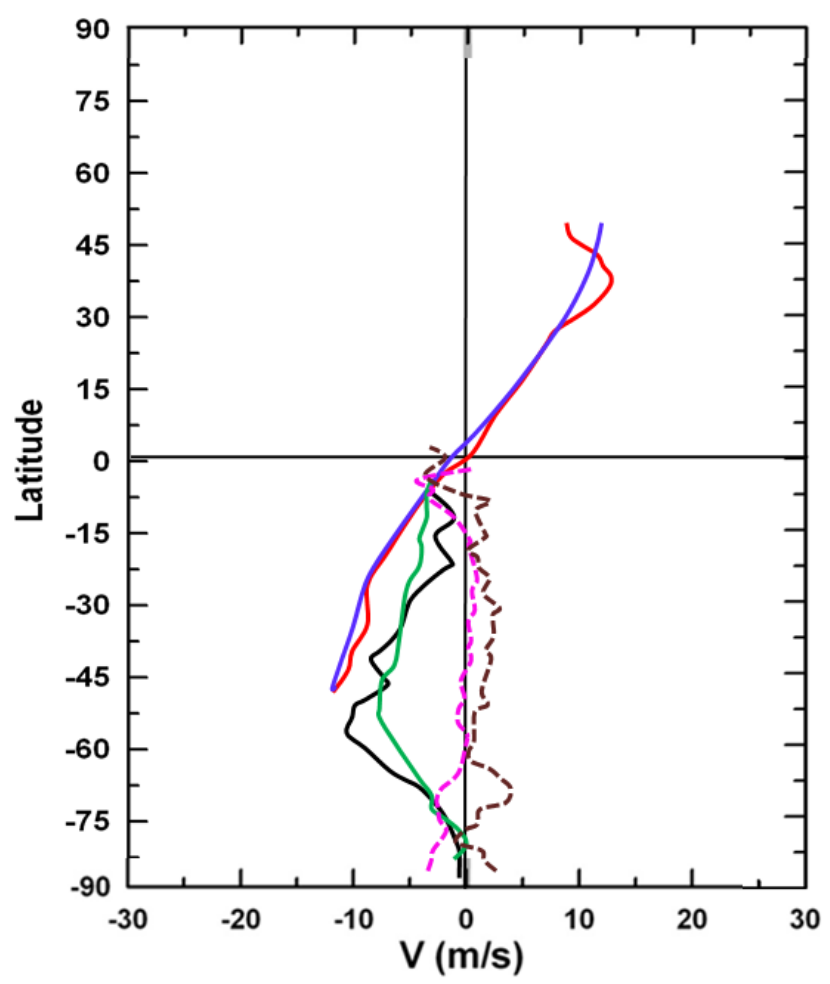

Figure 5. Meridional profiles of the mean North-South velocity as measured at different heights. (1)Upper cloud (65-70 km): Pioneer-Venus, 1979-1983 (blue line); Galileo, 1990 (red line); Venus Express VIRTIS, 2006-12 (black line); Venus Express VMC, 2006-12 (green line); (2) Altitude 60km: Venus Express VIRTIS, 2006-12 (dashed magenta); (3) Altitude 45-50 km in nighttime: Venus Express VIRTIS, 2006-12 (dashed brown).

\subsubsection{Vertical (upward-downward) winds.}

The vertical (upward-downward) wind velocity $w$ was directly measured by the Vega 1 and 2 balloons at an altitude level of $53 \mathrm{~km}$ during their longitudinal drift of about 11,000 km, dragged by mean zonal winds of $70 \mathrm{~m} \mathrm{~s}^{-1}$ at this altitude level (Blamont et al., 1986; Linkin et al., 1986). The atmospheric velocity fluctuated between -3 and $+3 \mathrm{~m} \mathrm{~s}^{-1}$ (Figure 6; $1 \mathrm{hr}$ corresponds approximately to a path of $250 \mathrm{~km}$ ). In addition vertical profiles of $w(z)$ have been retrieved 
during the descent of the Venera probes from direct measurements and from the descent aerodynamics that show that $w$ fluctuated between $\pm 1 \mathrm{~m} \mathrm{~s}-1$ between 0 and $60 \mathrm{~km}$ (Kherzanovich and Marov, 1983). These vertical velocities could originate from different processes (e.g. waves, in particular gravity waves excited by mountains; convective motions; Hadley cell circulations and topographically forced winds), and thus vary with altitude. Kerzhanovich and Marov (1983) and Gierasch et al. (1997) present calculations of the vertical profiles of $w(z)$, based on simple models of these processes, that predict slow velocities in Hadley cells $\left(w \sim 0.1 \mathrm{~m} \mathrm{~s}^{-1}\right)$ and moderate velocities for upward propagating waves and convective motions $\left(w \sim 0.5-5 \mathrm{~m} \mathrm{~s}^{-1}\right)$.

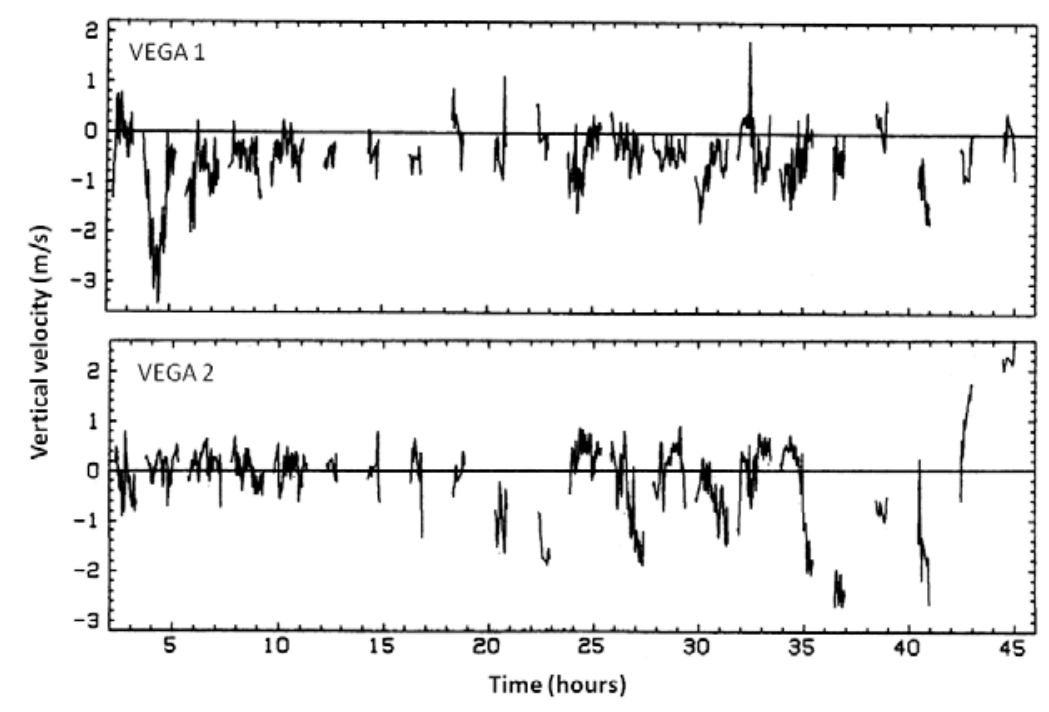

Figure 6. Vertical velocity at $\sim 53 \mathrm{~km}$ altitude level as measured by the balloons Vega 1 and 2 during their longitudinal drift by the zonal winds (approximately 1 hour $=252 \mathrm{~km}$ ). Adapted from Linkin et al. (1986).

\subsection{Wind variability}

Systematic studies of the variability of the winds have been performed using consistent time series of data from long duration orbital missions. The Pioneer Venus orbiter provided hemispheric long-term measurements of the zonal and meridional winds using cloud-tracking on the UV images provided by the OCCP instrument from 1979 to 1985 (Rossow et al., 1990; Limaye, 1987). Venus Express VIRTIS and VMC instruments provided wind data for the southern hemisphere from 2006 to 2012 (Kouyama et al., 2013; Khatuntsev et al., 2013; Hueso et al., 2015). The VIRTIS data cover at least two altitude levels, the upper cloud from UV images and the base of the cloud from images at $900-955 \mathrm{~nm}$.

\subsubsection{Dependences on Local Time}


The zonal and meridional $(u, v)$ components of the wind at the upper cloud $(65-70 \mathrm{~km})$ show an unambiguous dependence on the velocity with the local time (LT, or solar longitude) as shown by Rossow et al. (1990), Sánchez-Lavega et al. (2008), Kouyama et al. (2012), Khatuntsev et al. (2013) and Hueso et al. (2015). The precise, near simultaneous, independent and complementary studies using two different instruments on board Venus Express confirm this point (Figure 7). On one hand, the data show that, in the latitude band between $\sim 10^{\circ}$ and $40^{\circ}$, the zonal wind speed increases by $\delta u \sim 5-15 \mathrm{~m} \mathrm{~s}^{-1}$ from LT $=7 \mathrm{hr}$ to $17 \mathrm{hr}$, accelerating towards the local afternoon. This represents a signature of the action of the solar (thermal) tide on the wind field (see below). On the other hand, the meridional wind shows an increase of $\delta v \sim 10 \mathrm{~m} \mathrm{~s}^{-1}$ at LT $\sim 14-16 \mathrm{hr}$ in latitudes $\sim 40^{\circ}-50^{\circ}$, probably resulting from the combined action of the Hadley cell and the thermal tide (Peralta et al., 2012). There is no evidence of a dependence of the horizontal winds 500

501 on LT at the other two altitude levels where there are available data (60 km and $47 \mathrm{~km}$ ).
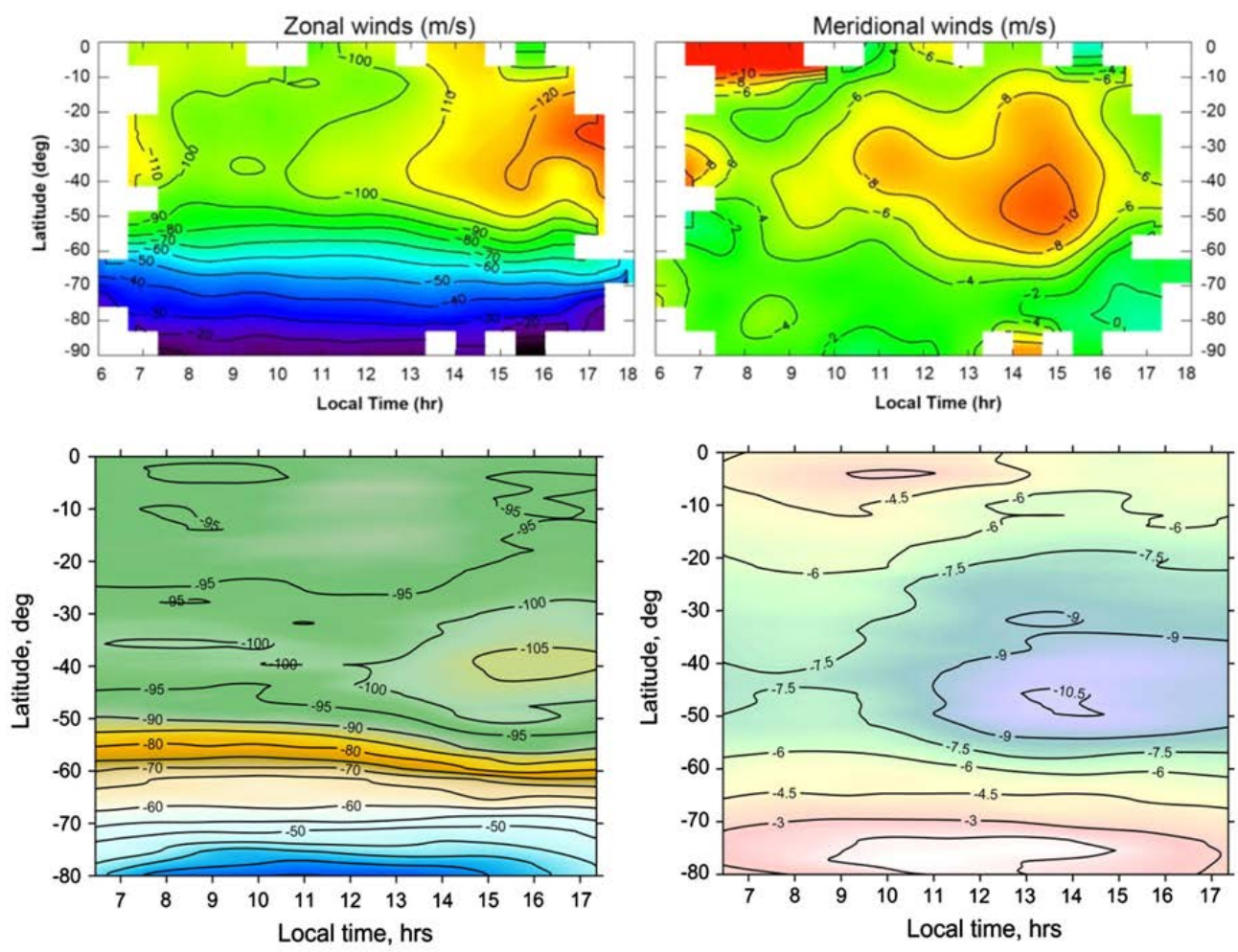

Figure 7. The long-term averaged horizontal wind velocity as a function of latitude and local time from the tracking of cloud motions with Venus Express. Upper panel: VIRTIS measurements 2006-12 (Hueso et al., 2014). Lower panel: VMC measurements (Kathuntsev et al., 2013).

\subsubsection{Short term variability}

The Venus Express VIRTIS and VMC analysis of the zonal velocity at the upper cloud level shows rapid fluctuations in its meridional profile on timescales of $\sim 24 \mathrm{hr}$ (the VEX orbital period) (Khatuntsev et al., 2013; Hueso et al., 1015). These fluctuations result from uncertainties in the measurements, the above described LT dependence (solar tide) and the presence of propagating waves (section 4). For example, Del Genio and Rossow (1990) and Rossow et al. (1990) identified oscillations in the zonal velocity with a period of 4.4 days and cloud brightness 
514 variability with a four and five day period; phenomena attributed to the presence of 515 wavenumber-1 Kelvin and Rossby waves, respectively. The presence of these waves was also 516 detected in Galileo wind data (Toigo et al., 1994; Peralta et al., 2007; Kouyama et al., 2012, 517 2013), and among them there is the conspicuous and semi-permanent feature known as the "Y", 518 "C" or "Psi"-wave due to the cloud morphology, recently interpreted by Peralta et al. (2015) as 519 an equatorially trapped Kelvin wave distorted due to the spherical geometry and to the fact that 520 the Kelvin wave phase extends to high latitudes.

521

522

523

524

525

526

527

528

529

530

531

532

533

534

535

536

537

\subsubsection{Long-term variability}

Inter-comparison between profiles of the mean zonal wind obtained by different missions since the 1970s reflects its long-term variability (Figure 2). The Pioneer Venus orbiter covered a long observing period (1979-1985), but no long-term drift in the wind field was detected (Rossow et al., 1990; Limaye, 2007). However, the data are suggestive of long-term multi-year cyclic variations in cloud top winds (Del Genio and Rossow, 1990, Rossow et al., 1990). Systematic studies of the zonal winds in the southern hemisphere, performed by the VEx instruments over 6 years, show a long-term global change in the zonal wind speed from equatorial to mid-latitudes (from $\sim-10^{\circ}$ to $-50^{\circ}$ ), accelerating from $u \sim-100 \mathrm{~m} \mathrm{~s}^{-1}$ (2006-2009) to $-120 \mathrm{~m} \mathrm{~s}^{-1}$ (2009-2012) (Khatuntsev et al., 2013; Hueso et al. 2015) (Figure 8). Since both independent studies correlate, the change could be real, although a sampling bias of the wind measurements toward particular solar local times, coupled to the solar tide acceleration, could also be involved in this result (Khatunsev et al., 2013). 


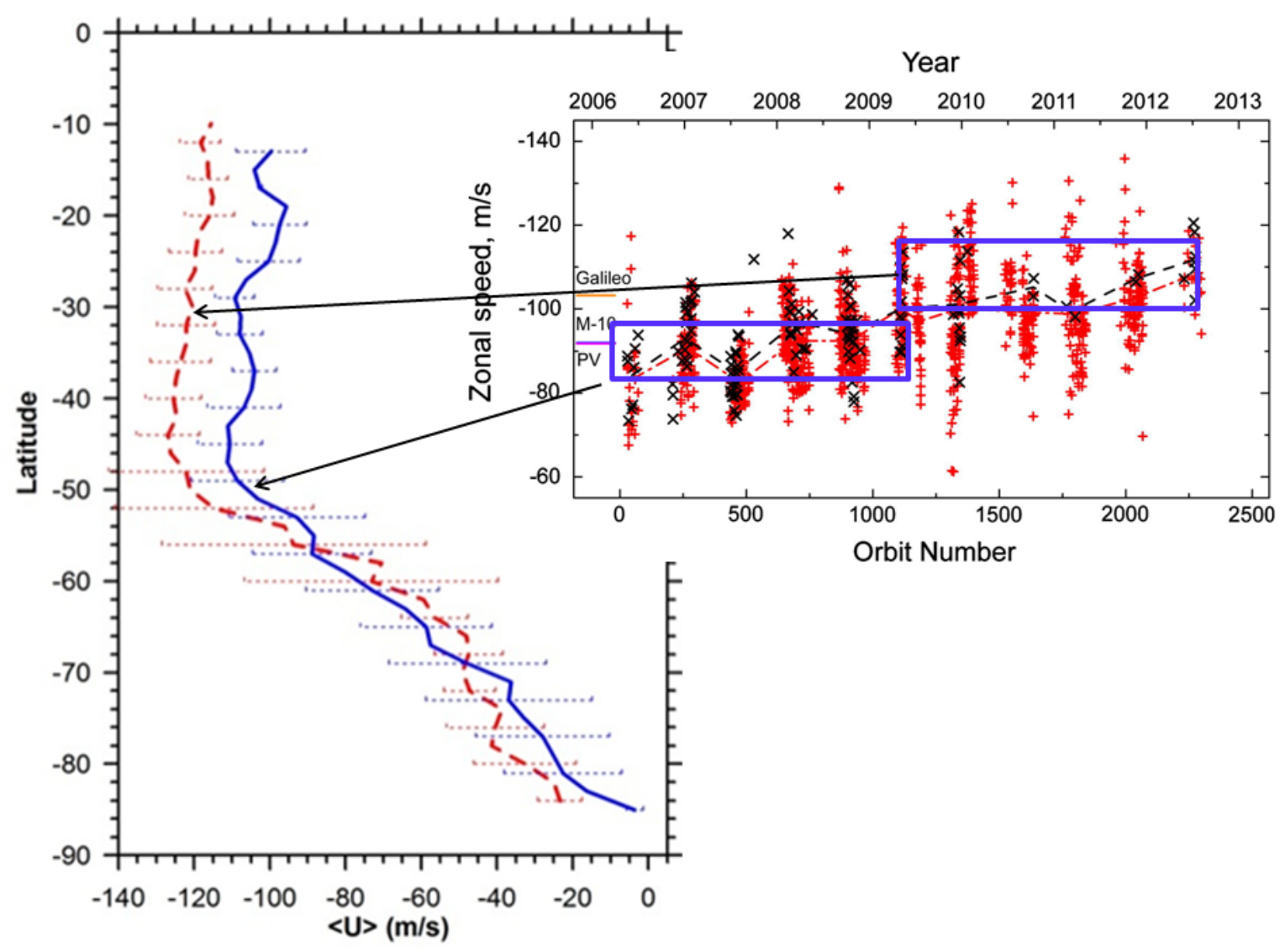

Figure 8. Long-term changes in the zonal wind velocity at 65-70 km altitude as measured by Venus Express instruments between local times 14-18 hrs. The left meridional profile of the zonal wind was obtained using VIRTIS in two periods 2006-09 (blue line) and 2010-12 (red dashed line) (from Hueso et al, 2014). On the right, averaged zonal wind speed is shown as a function of time obtained with VMC (from Khatuntsev et al., 2013). The blue boxes indicate the correspondence with the profiles at left.

\subsection{Upper atmosphere}

The region between the cloud tops at $\sim 65-70 \mathrm{~km}$ and the $110 \mathrm{~km}$ altitude level (a somewhat arbitrary frontier at $\mathrm{P}=2 \mu \mathrm{bar}$ ) is usually called the lower mesosphere. This is dynamically a transition region of complex dynamics that separates the Venus troposphere (dominated by the retrograde super-rotation, RSR) from the thermosphere, where a subsolar-antisolar (SS-AS) circulation cell dominates, driven by the solar EUV heating on the sunlit hemisphere (day-tonight flow). As indicated in section 2.1, the atmospheric motions in the altitude range between $z$ $\sim 70$ and $110 \mathrm{~km}$ are derived from the Doppler shift of CO absorption lines and $\mathrm{CO}_{2}$ non-LTE emissions and by tracking emission features from $\mathrm{NO}$ and $\mathrm{O}_{2}$ airglow (details can be found in reviews by Lellouch et al. (1997), Bougher et al., (1987), Limaye (2013 and references therein)). Indirectly, the wind field has been obtained from altitude-latitude measurements of the pressure 
and temperature fields and the direct application of either direct cyclostrophic balance or the thermal wind equation under such a balance (Seiff, 1983; Schubert, 1983, section 3 below).

562

$$
\underbrace{\frac{D v}{D t}}_{(1)}+\underbrace{\frac{u^{2} \tan \phi}{a}}_{(2)}+\underbrace{2 \Omega u \sin \phi}_{(3)}+\underbrace{\frac{1}{\rho a} \frac{\partial P}{\partial \phi}}_{(4)}=\underbrace{F_{\phi}}_{(5)}
$$

600 (2013).

\subsection{Thermal winds: Theory and Observations}

According to these measurements, the mesospheric dynamics is complex and globally dominated by the zonal retrograde flow, with decreasing winds with altitude from $70 \mathrm{~km}$ up to $\sim 85 \mathrm{~km}$ ( $50 \mathrm{~m} \mathrm{~s}^{-1}$ at mid-latitudes) according to cyclostrophic balance (Piccialli et al., 2012), but exhibiting very strong day to day variations, probably encountering the invasion of the SS-AS circulation in the upper part at $\sim 90-110 \mathrm{~km}$ (Lellouch et al., 1997; Widemann et al., 2007; Sornig et al., 2008; Limaye, 2013). Measurements in the 90-110 km altitude of the zonal component range from $\sim 5$ to $150 \mathrm{~m} \mathrm{~s}^{-1}$, whereas wind data for the SS-AS range from 40 up to $290 \mathrm{~m} \mathrm{~s}^{-1}$. Day-side and night-side winds are typically in the range $100-300 \mathrm{~m} \mathrm{~s}^{-1}$ (a stronger wind system at the evening terminator than the morning terminator) as summarized by Limaye

The motion of the morphology of $\mathrm{O}_{2}$ emission shows the complexity of the dynamics in the 90$110 \mathrm{~km}$ altitude range, with a zonal velocity dominated by an intense prograde jet (contrary to the retrograde planetary rotation) from dawn to midnight, with a reversed sign from dusk (Hueso et al., 2008). Typical zonal velocities range between +60 (eastward) to -50 (westward) $\mathrm{m} \mathrm{s}^{-1}$, while meridional velocities range from -20 (polewards) to $+100 \mathrm{~m} \mathrm{~s}^{-1}$ (equatorwards) with an average meridional drift of $+20 \mathrm{~m} \mathrm{~s}^{-1}$ towards low latitudes. All of these data on winds indicate the highly variable and complex nature of the motions in the transition layer. Further details on the dynamics of this region are presented in Chapter IX.

\section{Cyclostrophic balance and Angular Momentum}

In this section we provide a theoretical background to understand discussion about calculation of winds using assumptions of cyclostrophic balance (3.1), observed superrotation (3.2.2) and role of eddies (3.2.3), which are then detailed (in Section 4). A discussion of super-rotation mechanisms will be provided later in this chapter (section 8).

In common with other planetary atmospheres, the Venus atmosphere is governed by a combination of the Navier-Stokes equations, together with equations of continuity, thermodynamic energy conservation and a suitable equation of state. For most purposes, the Navier-Stokes equations are usually approximated by the meteorological primitive equations for a shallow atmosphere on the sphere, for which the meridional and vertical components are expressed as

$$
g+\frac{1}{\rho} \frac{\partial P}{\partial z}=0
$$


Here $\mathrm{u}$ and $\mathrm{v}$ are the zonal and meridional components of the velocity, $\mathrm{P}$ is pressure, $\phi$ is latitude, $\rho$ is density, $\Omega$ is the angular velocity ( $\mathrm{f}=2 \Omega \sin \varphi$ is the Coriolis parameter). The radius of the planet is denoted by $a$ and altitude above a reference geopotential is denoted by $\mathrm{z}$. $\mathrm{g}$ is the acceleration due to gravity and $t$ is time. Finally $F_{\phi}$ are other forcing sources. Equation (2) represents hydrostatic balance, which is a considerable simplification of the full vertical equation of motion but is a very good approximation for large-scale motion. For rapidly rotating planets, $\square$ is relatively large so that the dominant balance in Eq (1) is geostrophic (i.e. between terms (3) and (4)). For slowly rotating planets such as Venus, however, term (3) in Equation (1) is too small to balance the pressure gradient (term (4)) so that the centrifugal term (2) takes over. The resulting balance between terms (2) and (4) is known as cyclostrophic, as first identified by Leovy (1973), and can be stated (using (2) and pressure as a vertical "coordinate"

$u^{2}=-g \cot \phi\left(\frac{\partial z}{\partial \phi}\right) \frac{1}{\rho} \frac{\partial P}{\partial z}$

where the derivative represents the meridional slope of the constant pressure surface at $P$. For intermediate rotation rates, terms (2), (3) and (4) are of comparable magnitude and the resulting balance of these three terms is known as gradient wind balance.

By combining Equations (1) and (2) and the ideal gas law, a further equation can be derived for the vertical derivative of $u^{2}$. In pressure coordinates, this becomes

$627 \frac{\partial u^{2}}{\partial \varsigma}=-\frac{R_{g}}{\tan \phi}\left(\frac{\partial T}{\partial \phi}\right)_{P}$

where $\zeta=\ln \left(P / P_{0}\right)$, and $R_{g}$ and $T$ are the specific gas constant and temperature respectively, and is referred to as the cyclostrophic thermal wind equation.

642

These forms of cyclostrophic balance have been widely used indirectly to infer the mean zonal wind structure on Venus since the time of the early Venera and Pioneer Venus missions, using measurements of atmospheric temperature and pressure. The mesospheric temperature fields retrieved from infrared remote sensing instruments on board Pioneer Venus (Taylor et al. 1980), Venera-15 (Zasova et al. 2007), the Galileo Orbiter (Roos-Serote et al. 1995) and Venus Express (Grassi et al. 2010, 2014), together with radio occultation measurements from Pioneer Venus, the Venera-15 and Venus Express orbiters (Seiff 1980, Taylor et al. 1980, Lellouch et al. 1997, Zasova et al. 2007, Tellmann et al. 2009) have been used in various attempts to reconstruct Venus' zonal winds (Limaye 1985, Roos-Serote et al. 1995, Zasova et al. 2007, Piccialli et al. 2008, 2012, Mendonça et al. 2012). 
643

644

645

646

647

648

649

650

651

652

653

654

655

656

657

658

659

660

661

662

663

664

665

666

667

668

669

670

671

672

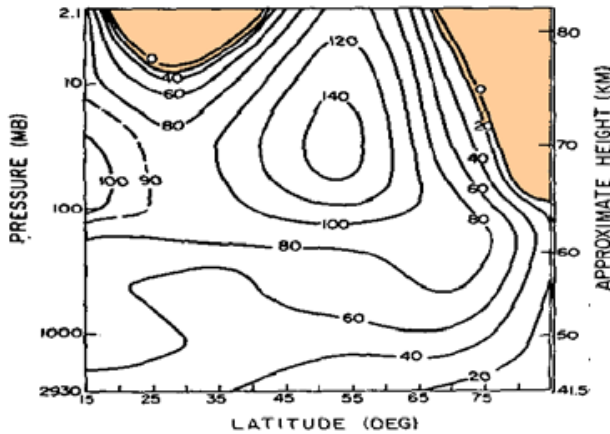

(a)

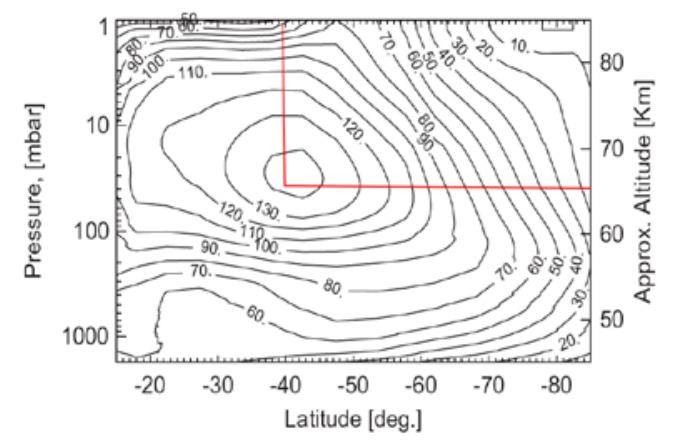

(b)

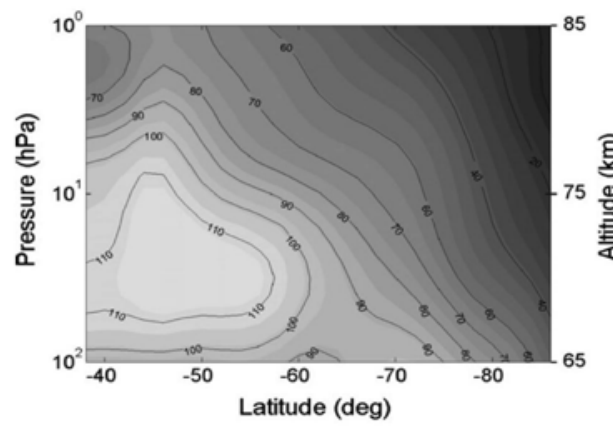

(c)

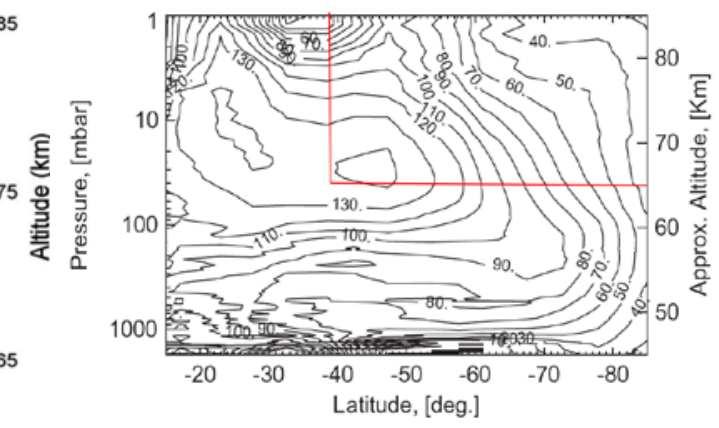

(d)

Fig. 9 Zonal velocity fields derived from integrating Eq (4) upwards from a given velocity profile: (a) from Pioneer Venus radio-occultation temperatures (Newman et al. 1984), (b) \& (c) from the VIRTIS instrument on the Venus Express spacecraft (Grassi et al 2010) by (b) Piccialli et al. (2012) and (c) Mendonça et al (2012); (d) zonal velocity field retrieved by direct application of Eq (3) using $\mathrm{z}(\mathrm{p})$ data from VeRA on Venus Express (Piccialli et al. 2012). Regions where no solution for $u$ is possible are shown shaded in (a). The region in (c) is indicated by red lines in (b) and (d) for comparison.

Most of these attempts have been based on a vertical integration of Eq (4) from an assumed latitudinal profile of zonal wind at the base of the domain, usually around the tops of the clouds at an altitude of 60-65 km. Some examples of zonal velocity fields reconstructed in this way are shown in Figure 9(a)-(c). Winds are typically seen to increase with height above the clouds towards peak values around $140 \mathrm{~m} \mathrm{~s}^{-1}$ in jet-like features at $\sim 40^{\circ}-50^{\circ}$ latitude and at altitudes of $\sim 70 \mathrm{~km}$. Secondary wind maxima are also sometimes inferred close to the equator, though these are less likely to be real because of increased sensitivity of $u^{2}$ to errors in $T$ associated with the $\tan \phi$ factor in Eq (4).

Such attempts to integrate Equation (4) in the vertical typically run into difficulties at high altitudes, however, especially near the equator and at high latitudes where the integration of (4) tends to force $u^{2}$ to become negative (e.g. see Figure 9a). This may happen either because of errors in the base wind profile or in the thermal field used in the integration, or because of a breakdown of the cyclostrophic balance approximation. Piccialli et al. (2012) showed that their reconstructions were particularly sensitive to errors in the base velocity profile around $70^{\circ}$ latitude, with the best results being achieved when using the best available observed wind profile 
from cloud tracking. Mendonça et al. (2012) also found a similar sensitivity to the base profile from comparisons with simple GCM simulations, which appeared to dominate over errors due to the possible breakdown of cyclostrophic balance. They were able to improve their reconstructions by using an iterative process that also involved correcting the upper level winds for self-consistency and further downward integration of (4) to correct the base profile.

Direct application of Equation (3) to $z(P)$ measurements, which may be available from radiooccultation observations, does not suffer from the amplification of errors in the temperature field or require an accurate base velocity profile. But reconstructions do suffer from effects of noise and sampling errors in the $z(P)$ measurements (see Figure 9(d)). With care, however, Figures 9(b)-(d) illustrates that reasonable agreement is possible between the two approaches.

The presence of a mid-latitude jet above the clouds appears to be a common feature in most balanced wind reconstructions, ranging from the Mariner 10 and Pioneer Venus missions through to Venus Express and using either Equation (3) or Equation (4). As is apparent from Figures 2 and 3 in Section 2, however, direct evidence for such mid-latitude jets from cloudtracking often seems elusive, although some profiles do seem to show a minimum of $\bar{u}$ on the equator and weak maxima at latitudes of $40^{\circ}-50^{\circ}$ in each hemisphere. This may indicate some significant variability in the structure of such jets with time, though this deserves further investigation in the future.

\subsection{Angular momentum conservation and transport}

In any atmospheric circulation system the flow must everywhere satisfy certain key conservation principles in relation to energy, momentum and angular momentum (AM). The latter two quantities are strictly vectors, but in the case of angular momentum, the axial component by itself may be conserved under certain circumstances. The axial component of absolute specific angular momentum, $m$, is defined in spherical polar coordinates as

$$
m=a \cos \phi(\Omega a \cos \phi+u)
$$

where $a$ is the planetary radius, $\Omega$ the planetary rotation speed, $u$ is the zonal velocity as measured in the frame rotating with the planet and $\phi$ is latitude. This expression is written for a shallow atmosphere, where the radial distance to the center of the planet $r$ is approximated to the radius $a$. In the zonal mean, the conservation equation for $m$ becomes

712 where the overbar represents the zonal mean, $\overline{\mathrm{u}}_{*}=\left(\bar{V}_{*}, \bar{W}_{*}\right)$ is the Transformed Eulerian zonal mean meridional velocity (e.g. Andrews et al. 1987), defined by 


$$
\bar{V}_{*}=\bar{v}-\frac{\partial}{\partial P}\left(\frac{\left(\overline{v^{\prime} \theta^{\prime}}\right)}{\partial \bar{\theta} / \partial P}\right)
$$

715

$$
\bar{w}_{*}=\bar{w}-\frac{1}{a \cos \phi} \frac{\partial}{\partial \phi}\left(\frac{\left(\overline{v^{\prime} \theta^{\prime}}\right) \cos \phi}{\partial \bar{\theta} / \partial P}\right)
$$

716

717

718

719

720

721

722

723

724

725

726

727

728

729

730

731

732

733

734

735

736

737

738

739

740

741

742

743

744

745

746

747 where $\theta$ is the potential temperature and primes denote departures from the zonal mean, and $E$ is a form of the Eliassen-Palm (EP) eddy flux of angular momentum, defined by

$$
\mathrm{E}=a \cos \phi\left\{\begin{array}{l}
\left(\overline{u^{\prime} v^{\prime}}\right)-\frac{\overline{\left(v^{\prime} \theta^{\prime}\right)} \partial \bar{u} / \partial P}{\partial \bar{\theta} / \partial P} \\
\left(\overline{w^{\prime} u^{\prime}}\right)+\left[\frac{1}{a \cos \phi} \frac{\partial}{\partial \phi}(\bar{u} \cos \phi)-2 \Omega \cos \phi\right] \overline{\left(v^{\prime} \theta^{\prime}\right)}
\end{array}\right\}
$$

and $F$ represents other residual torques e.g. due to friction or body forces.

For situations where the flow is frictionless and axisymmetric about the pole of the planet with no other body forces acting (so $F=0$ ), $\bar{m}$ is materially conserved following the motion in the meridional $(\phi, P)$ (or $(\phi, z)$ ) plane. This means that axial angular momentum per unit mass, contained in rings of fluid aligned along latitude circles, behaves like a materially conserved tracer. This leads to a number of important constraints, collectively referred to as "Hide's theorem” (Hide 1969, Schneider 1977, Held \& Hou 1980).

\subsubsection{AM conservation and role of eddies}

Once an axisymmetric ring of fluid has been initialised with a certain value of $\bar{m}$, there is no means of changing it in the absence of friction or body forces without breaking the axial symmetry. In the case of a planet like Venus, therefore, the fact that $u$ on the equator is observed to be in the same sense as the planetary rotation implies that $\bar{m}$ must exceed $\Omega a^{2}$, which is the maximum value of $\bar{m}$ for any point on the planet that co-rotates with the planet itself. Observing $\bar{m}>\Omega a^{2}$ must therefore require either special initial conditions or the action of nonaxisymmetric eddies (sometimes referred to as “Hide’s first theorem"; e.g. Read 1986; Read 2013). This has been demonstrated, for example, in simple GCM simulations under Titan-like or Venus-like conditions (e.g. see Mitchell \& Vallis (2010), their Fig. 2), where purely axisymmetric flows are unable to produce $u>0$ on the equator, even though prograde relative flow may occur at mid-high latitudes. In contrast, fully 3D flows with active eddies are able to produce equatorial super-rotation (as defined more fully below), much more like Venus itself.

This constraint also suggests ways of defining dimensionless quantitative measures of local and global "super-rotation", relative to an atmospheric state that co-rotates with the underlying 
planet:

$$
s=\frac{m-\Omega a^{2}}{\Omega a^{2}}
$$

750

$$
S=\frac{\iiint \rho m d V-M_{0}}{M_{0}}=\frac{\iiint \rho m d V}{M_{0}}
$$

where $M_{0}$ represents the integrated, mass-weighted, absolute angular momentum of the fluid with respect to volume $V$, in solid body co-rotation with the underlying planet (so $M_{0} \approx 8 \pi a^{4} \Omega P_{0} /(3 g)$ ) and $\rho_{m}=\rho u a \cos \phi$ is the relative angular momentum density. Thus, local and global superrotation can be defined respectively by $s>0$ or $S>0$.

\subsubsection{Quantifying the observed super-rotation}

With typical maximum zonal winds on the equator of up to $100 \mathrm{~m} \mathrm{~s}^{-1}$ or so (cf Figures 2 and 3; see Section 2), this suggests that Venus exhibits local values of $s$ of up to $\sim 60$, corresponding to the maximum ratio of the angular velocity of the zonal wind to that of the underlying planet. This represents the largest value of $s$ found in the atmosphere of any planetary body in the Solar $200 \mathrm{~m} \mathrm{~s}^{-1}$ on the equator; e.g. Flasar et al. 2009).

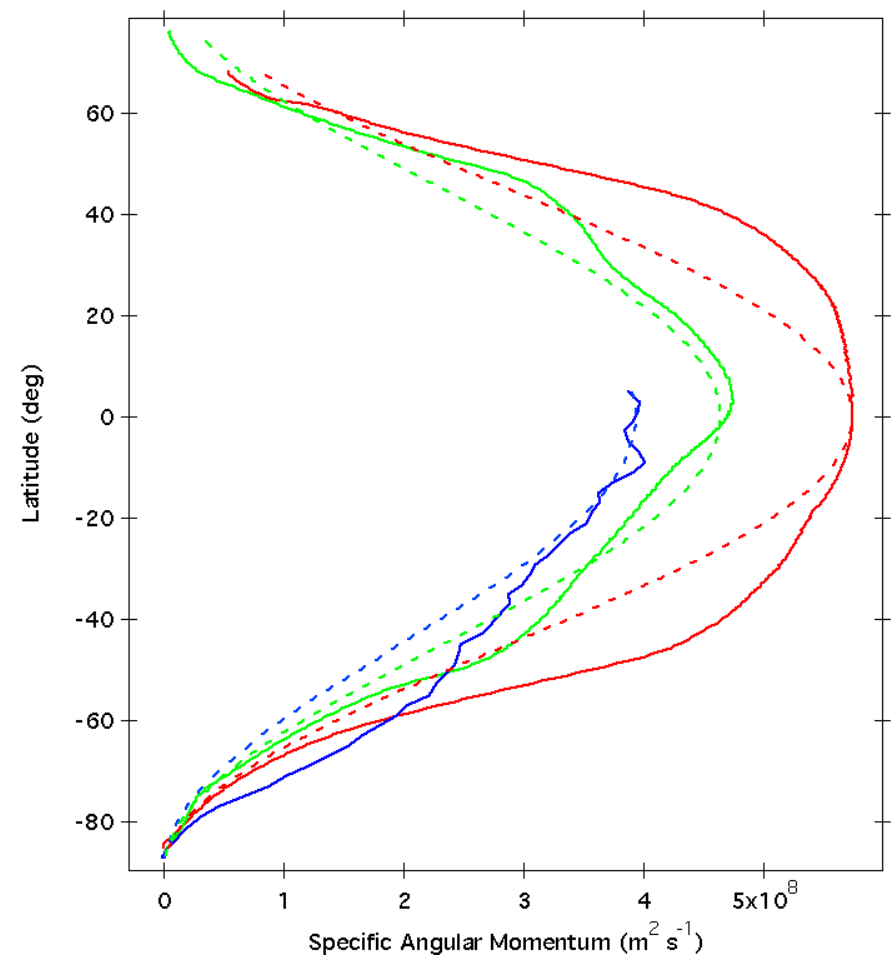


Figure 10. Latitudinal profile of specific angular momentum, $m$, corresponding to the set of cloud-tracked zonal wind profiles shown Figs 2 and 3, obtained from feature-tracking measurements at $U V$ wavelengths (solid red line; altitude $65 \mathrm{~km}$ ), $980 \mathrm{~nm}$ (solid green line; altitude $\sim 60 \mathrm{~km}$ ) and $1.74 \mu \mathrm{m}$ (solid blue line; altitude $\sim 45 \mathrm{~km}$ ). Dashed lines correspond to the profiles of $m$ for zonal winds in solid body rotation with their value at the equator (i.e. $m \sim$

Profiles of $m$ with latitude can be readily derived from winds measured e.g. using cloud-tracking from UV and IR images. Figure 10 shows such a profile derived from the wind measurements presented in Figures 2 and 3, in which the solid lines represent the mean values of the ensemble of profiles at each wavelength band in the UV (red), $980 \mathrm{~nm}$ (green) and $1.74 \mu \mathrm{m}$ (blue), corresponding to the approximate altitudes $65 \mathrm{~km}$ (blue), $60 \mathrm{~km}$ (green) and $45 \mathrm{~km}$ (blue). This clearly shows $m$ reaching a maximum value at low latitudes at all altitudes sampled, decreasing towards zero as both poles are approached. Even allowing for the variability represented in the ensemble of measurements, ranging from Mariner 10 to Venus Express VIRTIS and VMC, the low latitude peak in $m$ is quite broad and flat with relatively steep latitudinal gradients poleward of $40^{\circ}-50^{\circ}$ in each hemisphere, especially at the top of the main cloud deck in the UV profile. The dashed lines in Figure 10 indicate the profiles of $m$ (proportional to $\cos ^{2} \phi$ ) for a cloud level flow that would be in solid-body rotation with the zonal wind on the equator at each altitude level. This clearly shows the effect of poleward meridional transport in both hemispheres, tending to smooth out the low latitude peak and push the high equatorial values of $m$ to higher latitudes. This most likely represents transport by the upper branch of near-hemispheric Hadley circulations, as implied by the cloud-level meridional winds shown in Figure 5 and discussed in Section 2.3.1, in which $m$ is almost conserved as it is transported polewards from the equator, 794 

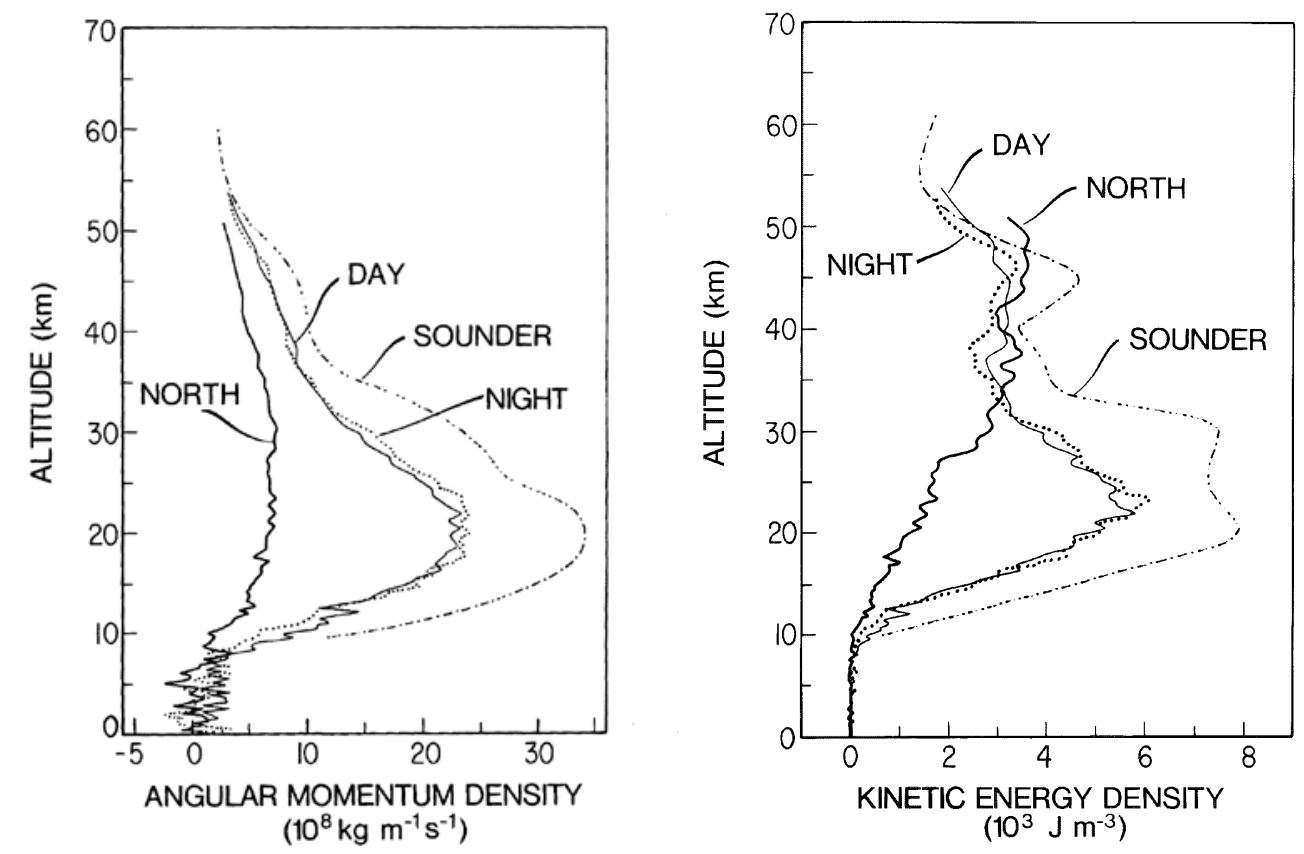

Figure 11 Observed vertical profiles of (a) angular momentum density $\rho_{m}=\rho u a \cos \phi$ and (b) kinetic energy density $\rho_{K}=\rho u^{2} / 2$, obtained by the Pioneer Venus entry probes (Schubert et al. 1980; with permission).

For the global super-rotation parameter $S$, the best available measurements of $\rho_{m}$ continue to be those obtained by the Pioneer Venus and Soviet Venera descent probes from the 1970s and 1980s (Schubert et al. 1980; Kerzhanovich \& Marov 1983; Kerzhanovich \& Limaye 1985). The

803 Pioneer Venus results for $\rho_{m}$ and kinetic energy density $\rho_{K}=\rho u^{2} / 2$ are shown in Figure 11, 804 representing profiles close to the equator ("Sounder") and at latitudes of $\sim 30^{\circ} \mathrm{S}$ ("Day" and "Night”) and $\sim 60^{\circ} \mathrm{N}$ ("North"). Similar profiles were obtained from tracking of the Venera 8-10 and 12-14 spacecraft during their respective descents, mostly between $13^{\circ} \mathrm{S}$ and $16^{\circ} \mathrm{N}$ apart from Venera 9 at $32^{\circ} \mathrm{N}$ (Kerzhanovich \& Limaye 1985).

809 These profiles clearly show that both $\rho_{m}$ and $\rho_{K}$ reach a peak in the lower atmosphere around an altitude of $\sim 20 \mathrm{~km}$, even though $u$ itself peaks much higher in the atmosphere. The amplitude of variations in both $\rho_{m}$ and $\rho_{K}$ also varies strongly with latitude, with the largest peak values located close to the equator and apparently decreasing towards either pole, where both quantities 813 must tend to zero. 
815

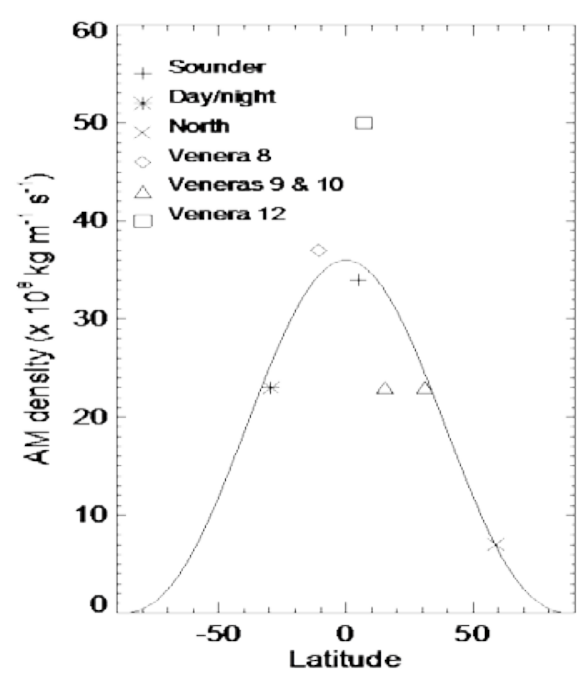

(a)

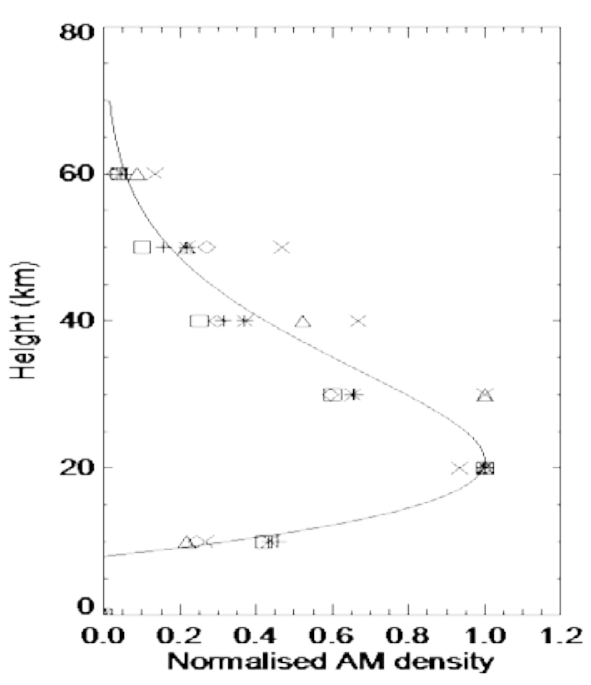

(b)

Figure 12 (a) Profile of the maximum values of $\rho_{m}$ with latitude and (b) profiles of $\rho_{m}$ with altitude, normalized by their respective maximum values, for Pioneer Venus and Venera 8-10 and 12 measurements. In (a) the solid line represents an empirical fit of the function $\rho_{m}=A \cos ^{5 / 2} \phi$ to these data, with $A=3.6 \times 10^{9} \mathrm{~kg} \mathrm{~m}^{2} \mathrm{~s}^{-1}$. The solid line in (b) represents an empirical fit to the function $\rho_{m}=\left(h-h_{0}\right) \exp \left[-\left(h+h_{1}\right)^{2} / b\right]$ where $h$ is in $\mathrm{km}, h_{0}=8 \mathrm{~km}, h_{1}=$ $25 \mathrm{~km}$ and $b=1200 \mathrm{~km}^{2}$.

Figure 12(a) plots the peak values of $\rho_{m}$ as a function of latitude from the Pioneer Venus and Venera probes. With the exception of two outliers (Veneras 10 and 12), these appear to lie close to a smooth variation with latitude that is roughly proportional to a function of the form $\rho_{m}=A \cos ^{\alpha} \phi$ with $\alpha \approx 5 / 2$ and $A \approx 3.6 \times 10^{9} \mathrm{~kg} \mathrm{~m}^{-1} \mathrm{~s}^{-1}$. Unlike $\rho_{K}$, which appears to peak at an altitude closer to $40-50 \mathrm{~km}$ around $60^{\circ}$ latitude according to the PV North probe, the observed vertical variations of $\rho_{m}$ lie close to a functional form with height that does not depend strongly on latitude. Figure 12(b) illustrates the measured profiles of $\rho_{m}$ from the Pioneer Venus and Venera probes, normalized by their respective maximum values. With the possible exception of the PV North probe, these normalized profiles mostly collapse onto an approximate form close to the empirical function $\rho_{m}=\left(h-h_{0}\right) \exp \left[-\left(h+h_{1}\right)^{2} / b\right]$, where $h$ is given in $\mathrm{km}$. The solid line in Fig. 12(b) represents a rough fit to these data with values of $h_{0}=8 \mathrm{~km}, h_{1}=25 \mathrm{~km}$ and $b=$ $1200 \mathrm{~km}^{2}$.

Given these approximate empirical functional fits to the latitudinal and vertical variations of $\rho_{m}$ across the planet, and (courageously!) assuming we can extrapolate these distributions to both poles, we can integrate $\rho_{m}$ across the entire planet to obtain a rough estimate of the global superrotation parameter $S$ from Eq (9). This leads to a value of $S \approx 8.2$, corresponding to a total angular momentum of $\sim 2.9 \times 10^{28} \mathrm{~kg} \mathrm{~m}^{2} \mathrm{~s}^{-1}$, indicating that the Venus atmosphere contains $\sim 8.2$ 
times more angular momentum that it would have if it was in solid body rotation with the underlying planet (for which $M_{0}=3.79 \times 10^{27} \mathrm{~kg} \mathrm{~m}^{2} \mathrm{~s}^{-1}$ ). Because of the crudeness of this estimate, the uncertainties on this quantity are quite large (at least $\pm 20-30 \%$ ) and it is also likely to be quite strongly variable in time (possibly accounting for the outliers in Figure 12a). But this estimate does serve to demonstrate the uniquely strong super-rotation of the Venus atmosphere, being rivaled only by Titan's atmosphere (which also substantially super-rotates; e.g. Flasar et al. 2009) and in complete contrast to the more rapidly rotating planets Earth and Mars, for which $S$ $<<1$ (and $s<<1$ ). The Venus atmosphere is evidently able to store and maintain massive quantities of angular momentum in such a way that it is kept largely isolated from the solid planet, despite the presence of viscous and form-drag torques at the planetary surface. Such massive values of $s$ and $S$ pose huge challenges to numerical models seeking to capture and simulate the dynamical state of the Venus atmosphere with quantitative precision, as discussed further in Section 8.2.

864

\subsubsection{Constraints on eddy AM fluxes}

The arguments leading to Hide's $1^{\text {st }}$ theorem, as outlined above, were further generalized by Schneider (1977) and Held and Hou (1980) to provide constraints on the properties of long term mean angular momentum transfers by eddy fluxes. Integration of Eq (6) over a region bounded by a closed $\bar{m}$ contour implies that

$$
\iint E \bullet d n=0
$$

(neglecting $F$ and enforcing mass conservation). This implies either that $E$ must be everywhere parallel to every $\bar{m}$ contour (which is highly unlikely) or have components that act both parallel to and anti-parallel to the gradient of $\bar{m}$ in different places, i.e. up-gradient eddy angular momentum fluxes are necessary to maintain a super-rotating flow. Hide's (second) theorem might then be stated as implying that an axisymmetric atmosphere cannot super-rotate at the equator if small-scale mixing of angular momentum is everywhere down-gradient.

In the original concept put forward by Gierasch (1975) for maintaining Venus's strong superrotation, the role of eddies was taken by a simple viscous-like diffusion that tends to transport angular momentum in the direction anti-parallel to angular velocity gradients (e.g. Plumb 1976; Read 1986; see also Section 8.1). Such diffusive processes, however, were required to be strongly anisotropic (i.e. much stronger in the horizontal than in the vertical) in order to balance realistic flows. They also required a very high turbulent Prandtl number (= ratio of momentum to thermal diffusivities) so that eddy heat fluxes did not destroy the large-scale thermal gradients necessary to achieve cyclostrophic balance with the observed winds.

If the role of eddy transport is taken by complex wave processes, however, eddy angular momentum transport is not constrained to behave in a diffusive manner with respect to any largescale variable. Up-gradient transport is therefore possible in principle in the vertical direction as well as in the horizontal. Thus, in general waves and eddies allow a wider range of possible 
configurations of $E$ than suggested in the original concept of Gierasch (1975). The details of how such wave-like eddies act in this context depends strongly on the type of waves that may be dominant in different parts of the atmosphere. These wave processes are now discussed in the next section.

\section{Wave Phenomena}

Atmospheric waves are created by a variety of mechanisms such as flow instabilities, diurnal heating variations, local convection, and flows over mountains on the Earth, and they play key roles in atmospheric dynamics. This should also apply to Venus: several types of waves are known to exist in the Venusian atmosphere and have been extensively studied with observations theories and numerical models.

Momentum transport by atmospheric waves is thought to be indispensable component of the general circulation of the Venusian atmosphere including the super-rotation. Hadley circulation carries superrotating angular momentum away from the equatorial region and cannot sustain the maximum of angular momentum that we observe at the equatorial cloud tops (Hou 1984). There must be non-axisymmetric motions that work for "returning-back" that angular momentum.Various types of waves have been proposed as candidates of such non-axisymmetric motions. Atmospheric waves are also thought to be a major source of turbulence, which induces diffusion of momentum, energy and atmospheric constituents to affect the vertical structure of the atmosphere.

We consider four types of waves observed in the Venus atmosphere: gravity waves, Kelvin waves, Rossby waves, and thermal tides. Although Kelvin waves and thermal tides can be classified as planetary-scale gravity waves, only small-scale waves are referred to as gravity waves here. We review waves other than the thermal tides in this section. Acoustic waves and Lamb waves have never been detected, although several works have predicted the characteristics of acoustic waves in the atmospheres of planets including Venus (Petculescu and Lueptow 2007).

\subsection{Wave observations: classification}

Wave phenomena are abundant in the Venus atmosphere and play a major role in atmospheric dynamics. These waves manifest in different forms: (a) in the cloud field, as regular brightness contrast patterns (Figure 13); (b) in the thermal field maps, as temperature oscillations (solar tide); (c) in the zonal wind, in the form of velocity oscillations as a function of latitude and local time (Figure 7); (d) in the vertical temperature profile, from radio-occultation measurements (Figure 14) and in situ descending probes; (e) in the infrared O2 nightglow emission from limb observations (Altieri et al. 2014) Figure 13 shows examples of waves in the cloud field, in particular gravity waves at two altitude levels and the planetary-scale wave known as the Y-or Psi- wave (Belton et al., 1976; Rossow et al., 1990; Del Genio and Rossow, 1990; Peralta et al., 2007, 2008; Piccialli et al., 2014). Figure 14 shows examples of the temperature fluctuations in the vertical, representative of gravity wave propagation (Jenkins et al., 1994; Hinson and Jenkins, 1995; Tellman et al., 2012). 
A

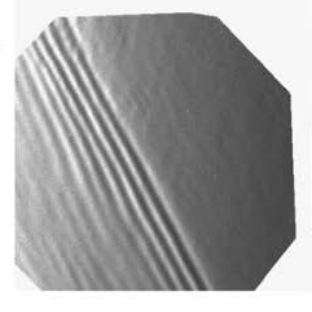

B

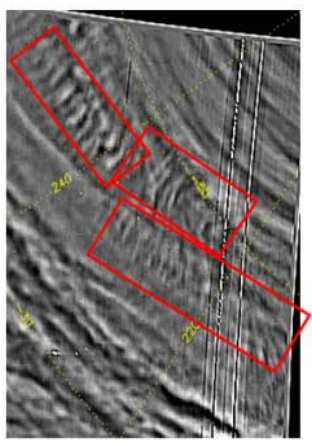

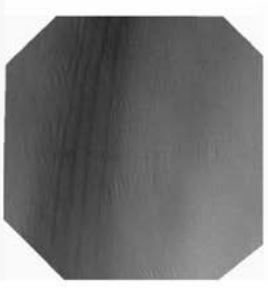

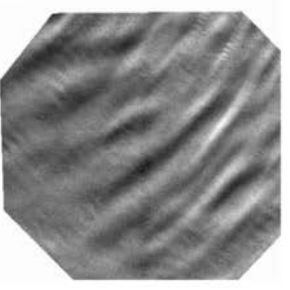

C 90

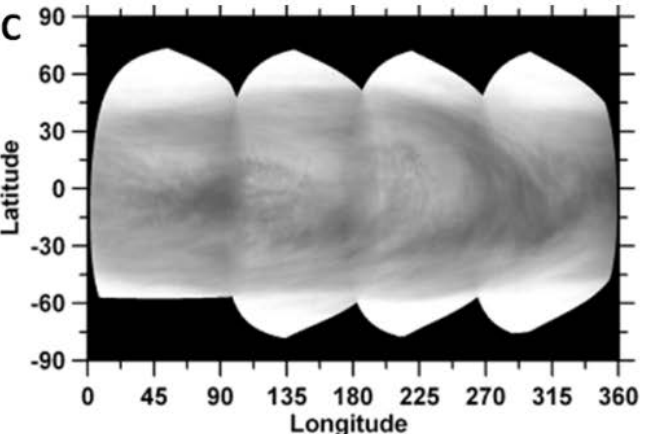

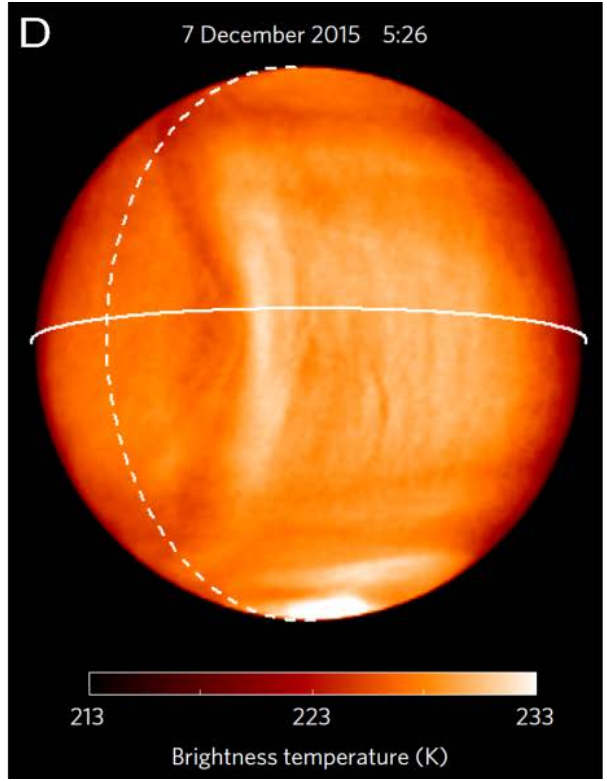

936

937

938

939

940

941

942

943

944

945

946

Figure 13. Wave manifestations at different cloud levels: (A) Gravity waves (short wavelength, tens of $\mathrm{km}$ ) in the upper cloud layer $(65-70 \mathrm{~km})$ from Venus Express VMC images (Piccialli et al., 2014); (B) Gravity waves (long wavelength, 100-300 km) in Venus's lower cloud layer (45$50 \mathrm{~km}$ )in nighttime from Venus Express VIRTIS images at $1.74 \mu \mathrm{m}$ (Peralta et al., 2008); (C) Map of the upper cloud at violet wavelengths showing the Y-or Psi-planetary-scale wave from Galileo flyby (Peralta et al., 2007); (D) Large stationary gravity wave in the upper cloud layer (65-70 km) from Akatsuki $10 \mu \mathrm{m}$ images (Fukuhara et al. 2017). 


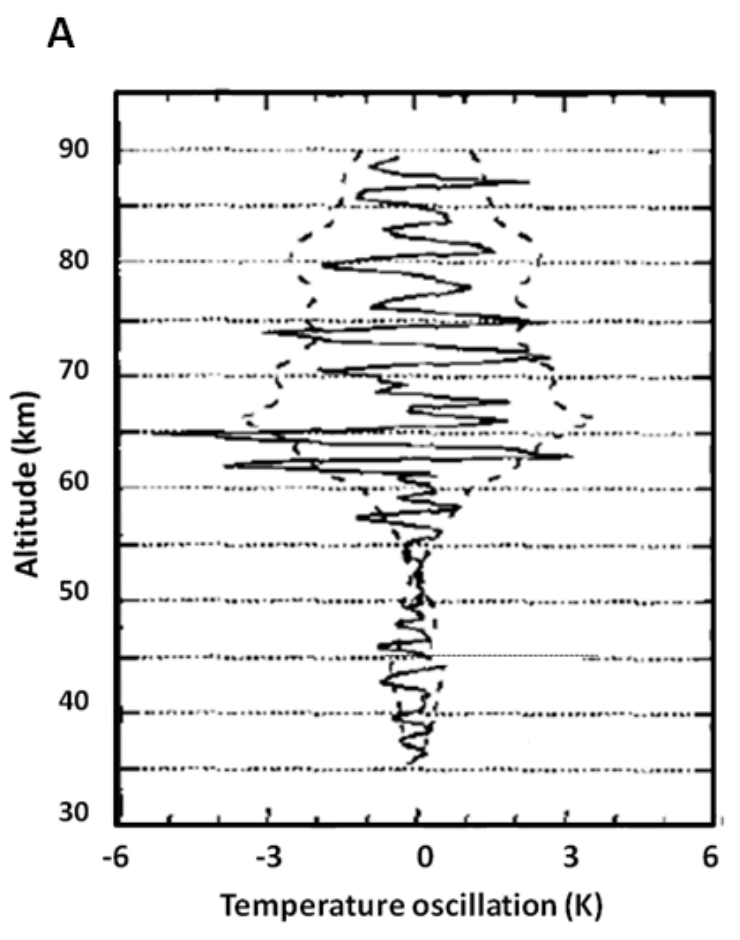
techniques.
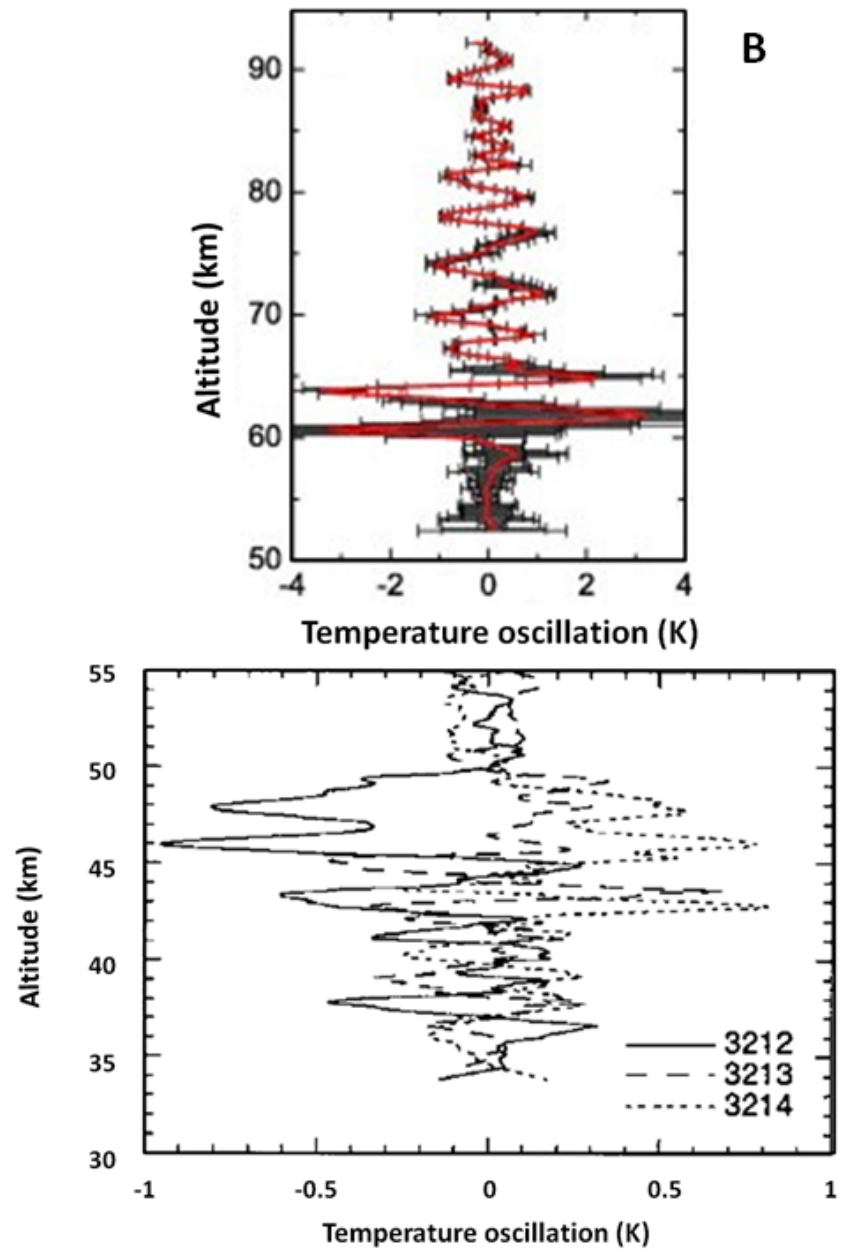

C

Figure 14. Vertical temperature oscillations from radio-occultation retrievals, revealing the presence of gravity waves with different amplitudes and wavelengths from 35 to $90 \mathrm{~km}$ altitude (A) Magellan spacecraft, Hinson and Jenkins (1995); (B) VERA instrument onboard Venus Express, Tellman et al. (2012); (C) Magellan spacecraft, Jenkins et al. (1994).

Table 2 summarizes the measured properties of the main observed waves by means of different

Table 2. Examples of observed waves and their measured properties in Venus atmosphere

\begin{tabular}{|c|c|c|c|c|c|c|c|c|}
\hline $\begin{array}{c}\text { Identification } \\
\text { Technique }\end{array}$ & $\begin{array}{c}\text { Wave } \\
\text { parameter }\end{array}$ & $\begin{array}{c}\mathbf{Z} \\
(\mathbf{k m})\end{array}$ & $\boldsymbol{A}$ & $\boldsymbol{n}$ & $\begin{array}{c}\boldsymbol{L}_{\boldsymbol{x}} \\
(\mathbf{k m})\end{array}$ & $\begin{array}{c}\mathbf{L}_{\mathbf{z}} \\
(\mathbf{k m})\end{array}$ & $\begin{array}{c}C_{X}-u \\
\left(\mathbf{m s}^{-1}\right)\end{array}$ & $\begin{array}{c}\text { Wave } \\
\text { type }\end{array}$ \\
\hline $\begin{array}{c}\text { Radio } \\
\text { occultation }\end{array}$ & $\mathrm{T}(\mathrm{z})$ & $40-90$ & $4 \mathrm{~K}$ & - & 2,800 & 2.5 & +0.4 & $\begin{array}{c}\text { Gravity }^{(1)} \\
\text { (vertical) }^{(2)}\end{array}$ \\
\hline Radiometry & $\mathrm{T}(\mathrm{LT})$ & $65-100$ & $5-10$ & 2 & & - & & Solar tide \\
\hline
\end{tabular}




\begin{tabular}{|c|c|c|c|c|c|c|c|c|}
\hline & & & $\mathrm{K}$ & & & & & \\
\hline UV Imaging & $\mathrm{u}(\mathrm{z})$ & $65-70$ & $\begin{array}{c}5-10 \\
\mathrm{~m} / \mathrm{s}\end{array}$ & 2 & - & - & - & Solar tide ${ }^{(3)}$ \\
\hline UV Imaging & $\begin{array}{l}\text { Brightness } \\
\text { contrast }^{(*)}\end{array}$ & $65-70$ & - & - & $60-150$ & - & +10 & Gravity $^{(4)}$ \\
\hline UV Imaging & $\begin{array}{l}\text { Brightness } \\
\text { contrast }^{(\#)}\end{array}$ & $65-70$ & & - & $3-21$ & - & & Gravity $^{(5)}$ \\
\hline UV Imaging & $\begin{array}{c}\text { Brightness } \\
\text { contrast Y- } \\
\Psi^{(+)}\end{array}$ & $65-70$ & - & 1 & 38,500 & & $\begin{array}{l}+5 \text { to } \\
+10\end{array}$ & $\begin{array}{l}\text { Kelvin }^{(6)} \\
\text { and } \\
\text { Kelvin- }^{-} \\
\text {Rossby }^{(7)}\end{array}$ \\
\hline $\begin{array}{l}\text { IR Imaging } \\
(1.7 \mu \mathrm{m})\end{array}$ & $\begin{array}{l}\text { Thermal } \\
\text { opacity } \\
\text { contrast }\end{array}$ & $45-50$ & - & - & 250 & & -10 & Gravity ${ }^{(4)}$ \\
\hline
\end{tabular}

963

964

965

966

967

968

969

970

971

972

973

974

975

976

977

978

979

980

981

982

983

984

985

986

987

988

989

990

991

992

Notes to Table 2: $z$ (altitude), $A$ (amplitude), $n$ (wavenumber), $L_{x}$ and $L_{z}$ (zonal and vertical wavelengths), $c_{X}-u$ (phase speed relative to mean zonal flow)

${ }^{(*)}$ Wave packets with extent: $\Delta L_{x}=950 \mathrm{~km}, \Delta L_{y}=350 \mathrm{~km}$

${ }^{(+)}$Period 4 days. Y- $\Psi$ shape in UV-images (equatorially oriented).

${ }^{(\#)}$ Wavefront (packet width): $50-400 \mathrm{~km}$

$\mathrm{LT}=$ local time

References to Table 2: (1) Hinson and Jenkins (1995); Jenkins et al. (1994); S. Tellmann et al. (2012); Häusler et al. (2006); (2) Schofield and Taylor (1983); Tellmann et al. (2009); (3) Del Genio and Rossow (1990); Rossow et al. (1990); Toigo et al. (1994); Smith and Gierasch (1996); Khatuntsev et al. (2013); Hueso et al. (2015); (4) Peralta et al. (2008); (5) Piccialli et al. (2014); (6) Belton et al. (1976), Peralta et al (2015); (7) Covey and Schubert (1982)

\subsection{Wave types and models}

A number of analytical and numerical studies have been conducted for the waves in the Venusian atmosphere. Linearizing the equations about the cyclostrophically-balanced basic state, the equations for the perturbation quantities resemble the ones for the terrestrial atmosphere (Covey and Schubert 1981; Peralta et al. 2014a). This suggests that the metric terms associated with the fast zonal wind takes the place of the Coriolis force; the dispersion relations used for the Earth have been used, considering a specific altitude region such as the cloud top (DelGenio and Rossow 1990). Numerical wave solutions based on the observed background wind distribution were also obtained (Covey and Schubert 1982; Schubert and Walterscheid 1984; Schinder et al. 1990; Smith et al. 1993; Kouyama et al. 2015). Waves found in general circulation models (GCMs) for Venus have also been classified into the existing categories (Yamamoto and Takahashi 2003; Lee et al. 2007; Lebonnois et al. 2010). Peralta et al. (2014a, b) obtained analytical solutions of various types of atmospheric wave on a planet in cyclostrophic balance with non-negligible latitudinal shear of the background zonal wind, and demonstrated that the wave type of each observed wavelike feature can be identified, based on its horizontal wavelength and intrinsic phase velocity (Figure 15). 


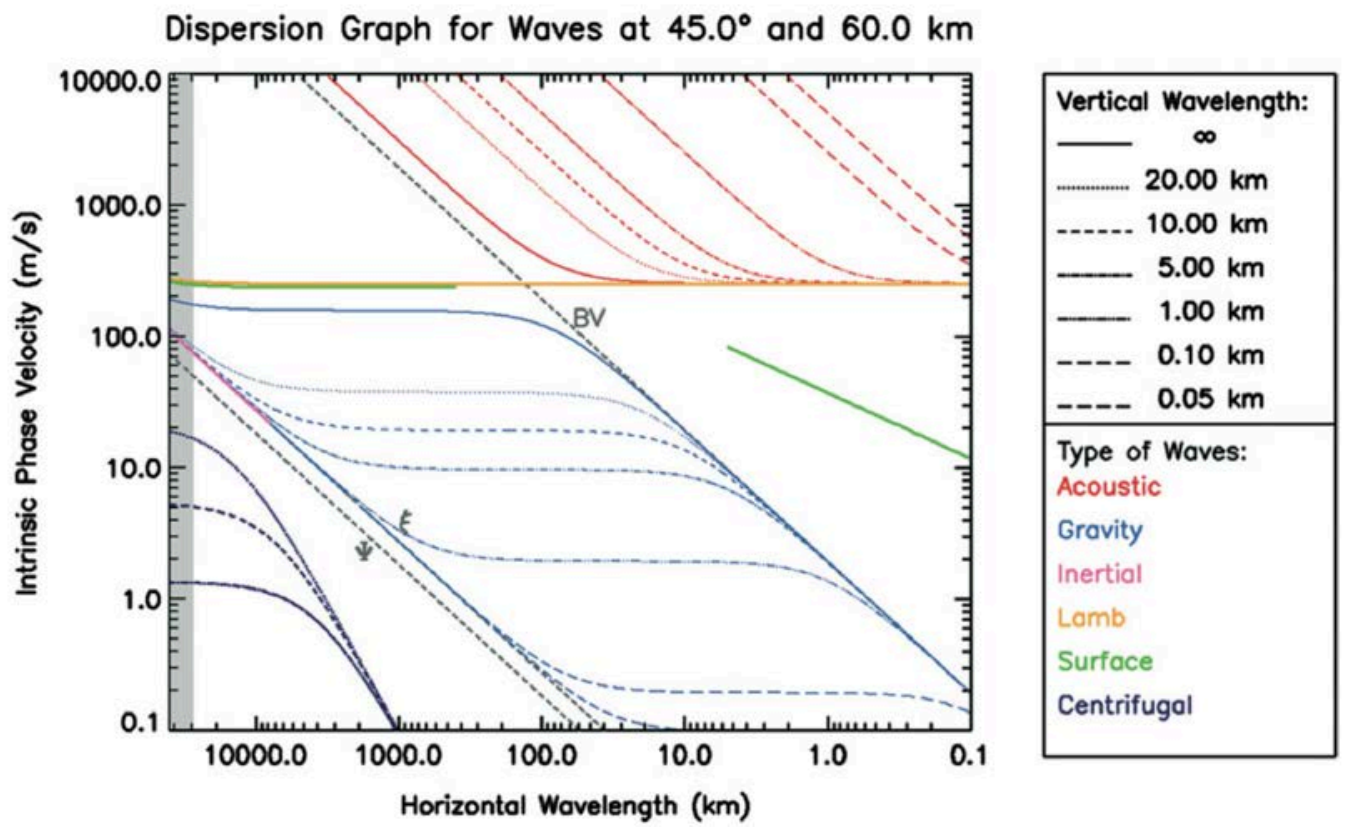

993

994

995

996

997

998

999

1000

1001

1002

1003

1004

1005

1006

1007

1008

1009

1010

1011

1012

1013

1014

1015

1016

1017

1018

1019

1020

1021

1022

Figure 15. Dispersion relations for acoustic, gravity, inertial, Lamb, surface, and centrifugal (Rossby) waves of Venus at a latitude of $45^{\circ}$ and an altitude of $60 \mathrm{~km}$. The Brunt-Väisälä frequency $(B V)$, centrifugal frequency $\left(\Psi=\left(u_{0} / a\right)\right.$ tan $\phi$, where $u_{0}$ is the background zonal wind, $a$ is the planetary radius, $\phi$ is the latitude), and centrifugal frequency modified by the meridional shear of the background zonal wind $\left(\xi^{2}=2 \Psi\left(\Psi-d u_{0} / d y\right)\right.$, where $y$ is the meridional distance) are marked with gray lines (Figure after Peralta et al. 2014b).

\subsubsection{Gravity waves}

Gravity waves are a type of atmospheric wave for which buoyancy serves as the restoring force. The overall stable stratification of the Venusian atmosphere should allow vertical propagation of gravity waves, except in the neutrally-stable middle cloud region from roughly 48 to $55 \mathrm{~km}$ altitude, the weak stability region from roughly 18 to $30 \mathrm{~km}$ altitude, and the boundary layer which likely exists adjacent to the surface (Seiff 1980; Tellmann et al. 2009). Gravity waves are considered to have relatively short horizontal and vertical scales in most cases; small-scale wavy features indicative of gravity waves were observed in cloud images and temperature/density profiles as shown in section 4.1 (Figures 13-14).

The regions of neutral or weak stability mentioned above are probable sites of thermal convection; penetration of convective plumes into the overlying and underlying stable layers is a plausible wave generation mechanism. Baker and Schubert (2000a, b) studied characteristics of sub-cloud gravity waves that are generated by convection in the middle cloud region at 48-55 km altitudes and in the lower atmosphere below $30 \mathrm{~km}$ altitude. The waves have horizontal wavelengths of $20-30 \mathrm{~km}$, which are comparable to the scales of convection cells. Eastwardpropagating waves generated by cloud-level convection were shown to experience critical level absorption below the cloud and decelerate the westward super-rotation there. Leroy and Ingersoll (1995, 1996) obtained analytical solutions for the spectrum of upwardly-propagating gravity waves generated by cloud-level convection, and compared the solutions with radio scintillation spectra. Imamura et al. (2014) conducted numerical experiments of cloud-level convection and 
1023

1024

1025

1026

1027

1028

1029

1030

1031

1032

1033

1034

1035

1036

1037

1038

1039

propagation of gravity waves both above and below clouds (Figure 16), and showed that wave generation is relatively suppressed in low latitudes and on the dayside due to the stabilizing influence of solar heating on the atmosphere; this suggested latitudinal trend is consistent with the results of Venus Express radio occultations showing that the gravity wave amplitude above clouds is larger at higher latitudes (Tellmann et al. 2012). Linear model studies showed that vertically-trapped, ducted waves with horizontal scales of tens of kilometers can be generated by cloud-level convection and that such waves can transport zonal momentum toward the equator to sustain the super-rotation (Gierasch 1987; Schinder et al. 1990). Venus Express radio occultations showed enhancement of the wave amplitude near the subsolar point as compared to other local times in low latitudes (Tellmann et al. 2012), suggesting additional wave excitation by mesoscale processes that are observed as cell-like features near the subsolar cloud top in ultraviolet images (Toigo et al. 1994). Gravity waves generated by boundary-layer convection are expected to have large influences on the zonal wind through critical level absorption (Schubert and Walterscheid 1984; Hou and Farrell 1987), although the nature of convection near the surface is poorly constrained.

1040

1041

1042

1043

1044

1045

1046

1047

1048

1049

1050

1051

1052

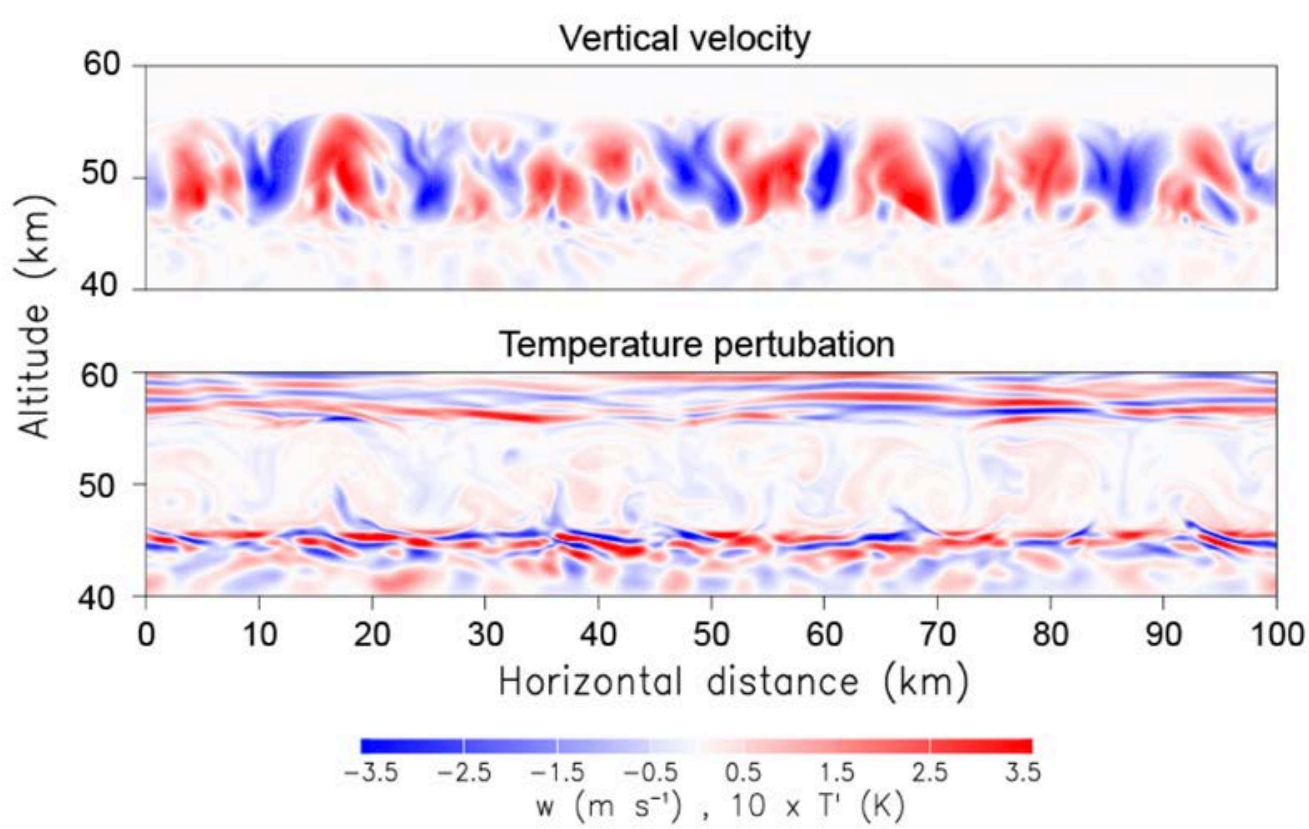

Figure 16. Snapshot of (upper) vertical velocity and (lower) temperature perturbation at midnight in a two-dimensional model for the diurnal cycle of the cloud-level convection at latitude $60^{\circ}$. Propagation of gravity waves in the stable layers above and below the convective region at 48-55 $\mathrm{km}$ altitudes is evident in the temperature perturbation. (figure after Imamura et al. 2014)

Given the observations of enhanced wave activity over large topographic features (Sagdeev et al. 1986; Titov et al. 2012; Tellmann et al. 2012), flows over topography should also be a major wave source. Bertaux et al. (2016) claim to have discovered a notable correlation of the cloudtop zonal wind speed with the underlying topography in the equatorial region and attributed the correlation to the vertical momentum transport by topographically-generated gravity waves. Fedorova et al. (2016) found that mesospheric water vapor is depleted above Aphrodita Terra via 
Venus Express spectroscopic measurements and that the correlation can be associated with the influence of the topography. More recently, inter-hemispheric bow-shaped structures were detected at the cloud tops by Akatsuki spacecraft and found to be fixed in position above the highlands (Fukuhara et al. 2017). Those bow-shaped structures might be topographicallygenerated gravity waves. The phenomenon is not yet reproduced by the LMD-GCM, which includes topography (see section 8.2), and would require detailed modeling for wave generation (and propagation) by the well known topography of Aphrodite Terra, the proposed source for the waves. Numerical models showed that waves generated near the surface can reach large amplitudes at cloud levels for forcing amplitudes representative of those expected in mountainous regions (Young et al. 1987, 1994). We should note, however, that the wind speed near the surface is very slow according to the measurements by Venera probes (Kerzhanovich and Marov 1983), although Aeolian features which could be explained as generated by persistent superrotating surface winds exist (Greeley et al. 1995). Shear instability beneath the mid-latitude jets can also generate gravity waves (Imamura 1997) and may partly explain the high-latitude enhancement of the mesospheric wave activity.

In an atmosphere where the static stability and the background wind velocity changes with height, gravity waves undergo trapping, resonance, evanescence and critical-layer absorption (Schubert and Walterscheid 1984; Young et al. 1987; Hou and Farrell 1987). Once the waves reach large amplitudes, wave breaking occurs, and nonlinear wave-wave interactions can excite secondary waves (Young et al. 1994). Wave breaking induces turbulence generation, leading to diffusion of energy, momentum and atmospheric constituents. Evidence of wave breaking has not been observed so far in the Venusian atmosphere except for the apparently 'saturated' spectra obtained from radio occultation temperature profiles (Ando et al. 2015). Strong radiative damping due to the $\mathrm{CO}_{2}$ atmosphere might effectively suppress amplitude growth with height and inhibit wave breaking, depending upon the frequency and the vertical wavelength of the wave (Hinson and Jenkins 1995).

Momentum exchange between different atmospheric layers via gravity waves generally acts to reduce the velocity difference between the two regions, and thus gravity waves are not expected to help maintaining the super-rotation. A different idea was proposed by Hou and Farrell (1987), who argued that the excitation of a continuous spectrum of gravity waves near the surface and the attenuation of the waves at different heights, depending upon their phase velocities, can provide retrograde (westward) acceleration below $45 \mathrm{~km}$ altitude. At thermospheric heights, friction supplied by the momentum deposition by gravity waves is expected to decelerate the subsolar-to-antisolar circulation, otherwise supersonic flow would occur (Seiff et al. 1982). Dynamical modeling of the thermosphere has shown that local-time asymmetry of the momentum deposition by gravity waves might explain the observed super-rotational component of the thermospheric circulation (Alexander 1992; Zhang et al. 1996).

\subsubsection{Kelvin wave}

A wavenumber-1 ultraviolet albedo feature is seen to move westward in low latitudes at a velocity close to the super-rotation, i.e. with a recurrence period of 4-5 Earth days (DelGenio and Rossow 1990). This albedo feature accompanies an oscillation of the equatorial zonal wind (Rossow et al. 1990; Smith et al. 1992; Kouyama et al. 2012, 2013). This 'four-day wave' is 
considered to be an equatorial Kelvin wave (Covey and Schubert 1981; DelGenio and Rossow 1990), which is a type of planetary-scale gravity wave that propagates in the direction of planetary rotation and is trapped near the equator, thanks to the rotational frame provide by the super-rotation itself. The e-folding latitude of the amplitude is estimated to be $20^{\circ}-30^{\circ}$ from the dispersion relation (DelGenio and Rossow 1990) and the observed wind structure (Kouyama et al. 2012). If the dark material responsible for the albedo variation is located just below the cloud top, as is commonly assumed (Esposito 1980; Pollack et al. 1980), the propagating dark region should correspond to a phase of the wave where air parcels are elevated (DelGenio and Rossow 1990).

The Kelvin wave is thought to have a vertically-propagating character with a vertical wavelength of 6-8 $\mathrm{km}$. The dissipation of the wave near the cloud top through radiative damping should therefore induce an equatorial acceleration (DelGenio and Rossow 1990; Imamura 2006; Kouyama et al. 2015). Yamamoto and Tanaka (1997) simulated the development of superrotation by a Kelvin wave-induced acceleration by forcing a Kelvin wave at the bottom boundary of a middle atmosphere dynamical model. Note, however, that the excitation region of the wave on Venus is unknown and that the vertical extent of the momentum transport is uncertain. Linear model studies suggest that a Kelvin wave and a hemispherically-symmetric Rossby wave, both having a zonal wavenumber of unity, can be excited even by random forcing as resonant modes of the whole atmosphere (Covey and Schubert 1982). Smith et al. (1992, 1993) argued that radiative-dynamic cloud feedback near the cloud base can excite a Kelvin wave as an unstable mode (Figure 17): vertical motions associated with a large-scale wave cause evaporation or condensation of cloud particles, which changes the infrared heating at the cloud base, leading to energy input to the wave. Iga and Matsuda (2005) showed, using the shallow-water equations, that the observed latitudinal profile of the zonal wind at the cloud top having mid-latitude jets is unstable and generates a Kelvin wave. This Kelvin wave accompanies equatorward transport of zonal (retrograde) momentum, and the resultant equatorial acceleration might contribute to the maintenance of the super-rotation. An analytical study showed that Kelvin waves can cause equatorward transport of zonal momentum also through interaction with the Hadley circulation (Imamura et al. 2004). It was also suggested that the vertical shear of the zonal wind below the cloud top forces Kelvin waves to focus equatorward with height, and that the resultant tilted EP 

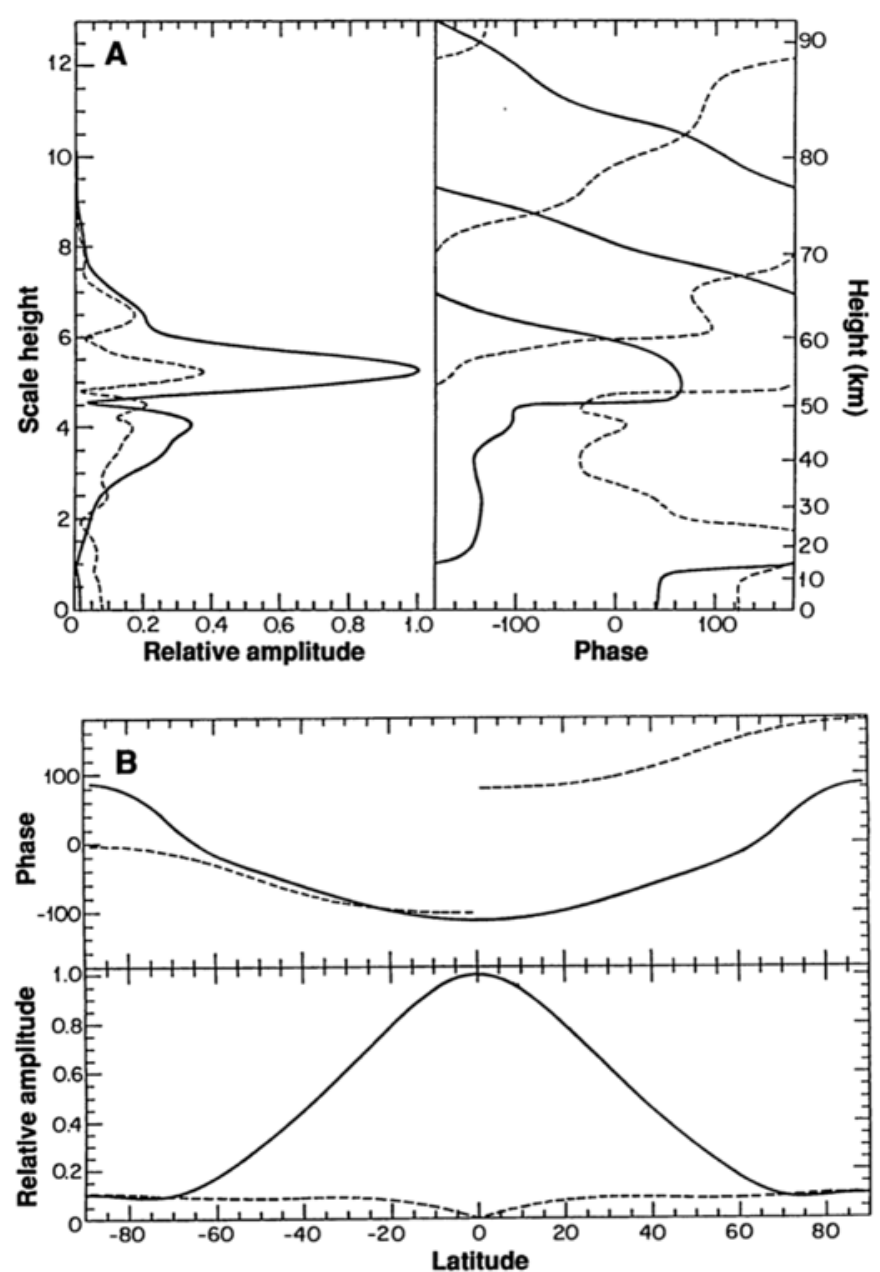

Figure 17. Structure of the most unstable wave mode in the Venus atmosphere obtained by a linear model with radiative-dynamic cloud feedback. The solid line shows zonal velocities and the dashed line shows meridional velocities. In (A) the zonal velocity is taken at the equator, and the meridional velocity is taken at $30^{\circ}$ north. The amplitude of the meridional velocity component has been multiplied by ten. In (B) both profiles are taken at $63 \mathrm{~km}$ altitude (figure after Smith et al. 1992).

The horizontal structure of the Kelvin wave might explain the global-scale horizontal "Y" feature (Smith et al. 1993) seen regularly at ultraviolet wavelengths at the cloud top from space missions (e.g. Belton et al. 1976; Schubert 1983; Rossow et al. 1980; Peralta et al. 2007, Titov et al. 2012) and from ground-based imaging (Sánchez-Lavega et al., 2016). The combination of the Kelvin wave and a hemispherically-symmetric Rossby wave could be essential in developing the Y feature, including the dark mid-latitude bands (Covey and Schubert 1982; Yamamoto and Tanaka 1997, Lee et al., 2010). Peralta et al. (2015) reproduce most of the observed peculiarities of the $\mathrm{Y}$ feature with a distorted Kelvin wave model in a cyclostrophic environment. 


\subsubsection{Rossby waves}

A wavenumber-1, hemispherically-symmetric ultraviolet albedo feature with maximum amplitudes occurring in the mid-latitudes is moving westward with a recurrence period of 5-6 Earth days (DelGenio and Rossow 1990). This "five-day wave" has been observed also in the wind field (Rossow et al. 1990; Kouyama et al. 2013). Based on the observed horizontal structure and the frequency, this mode is identified as a hemispherically-symmetric, gravest Rossby wave. Rossby waves can exist in a rotating fluid in spherical geometry because the restoring force is provided by the latitudinal gradient of the Coriolis parameter; on Venus the super-rotation of the atmosphere takes the place of the planetary rotation. Linear models suggest that the observed Rossby wave is a preferred mode of the Venusian atmosphere and can be excited even by random forcing similarly to the Kelvin wave (Covey and Schubert 1982). We should note, however, that Venus' atmosphere does not rotate as a solid body; the meridional shear of the background wind severely affects the beta-plane approximation, and thus the characteristics of Rossby-type waves are altered (Peralta et al. 2014b).

Rossby waves and mixed Rossby-gravity waves having a broad range of frequencies and wavenumbers are generated in Venus-like GCMs, principally through barotropic instability of mid-latitude jets (Yamamoto and Takahashi 2003; Lee et al. 2007; Lebonnois et al. 2010). Some of these waves seem to transport zonal (retrograde) momentum equatorward, thereby sustaining the super-rotation. Such a momentum transport is not self-evident; terrestrial Rossby waves, excited in the mid-latitude baroclinic zones, principally transport eastward zonal momentum poleward to strengthen the mid-latitude jet and decelerate the equatorial atmosphere. Mitchell and Vallis (2010) suggested that the transition of the wave momentum flux occurs when the Rossby deformation radius exceeds the planetary radius; this condition is easily satisfied on slowly rotating planets like Venus. On the other hand, analyses of cloud-tracked winds suggest predominantly poleward eddy momentum fluxes on the dayside (Limaye et al. 1982; Rossow et al. 1990). Observations of waves at lower altitudes and on the nightside are needed to test these theories.

The Rossby wave observed at the cloud top is considered to have a vertically-propagating character with a vertical wavelength of 20-30 km (Taylor et al. 1980; DelGenio and Rossow 1990). The radiative damping of the wave above the clouds should exert a drag force on the zonal flow in the middle and high latitudes (Imamura 2006); this deceleration might explain the observed decrease of the mean zonal wind above the cloud top (Newman et al. 1984) and can induce a wave-driven meridional circulation in the mesosphere, similar to the Brewer-Dobson circulation in the terrestrial middle atmosphere (Imamura 1997). Kouyama et al. (2015) suggested, based on linear wave solutions, that a competition between the mean-wind acceleration by the cloud-top Kelvin wave and the deceleration by the Rossby wave might be responsible for the observed year-to-year variability of the mean zonal wind.

\section{Thermal tides}

A major fraction of the solar energy incident on Venus is absorbed by the 'unknown' absorber and sulfuric acid aerosols in the upper cloud region above $58 \mathrm{~km}$ altitude (Tomasko et al. 1980). 
This unusual distribution of the absorbed energy generates planetary-scale gravity waves called thermal tides: the relative motion between the super-rotating atmosphere and the spatial pattern of the solar heating, which has a maximum in the sub-solar region, drives thermal tides with prograde (eastward) intrinsic phase velocities. The excited waves propagate both upwards and downwards from the forcing region. Because of the wealth of information obtained so far on the thermal tides as compared to other types of waves, we review this wave type separately in this 1204 section.

\subsection{Observations}

Thermal tides at the cloud top with zonal wavenumbers 1 (diurnal), 2 (semidiurnal) and higher were first observed in the thermal infrared using ground-based telescopes (Apt et al. 1980). In the temperature field in the Northern low latitudes, obtained by the infrared spectrometer on Pioneer Venus (Schofield and Taylor 1983), the vertical propagation of the semidiurnal tide is evident in the altitude region from the cloud tops to $100 \mathrm{~km}$ altitude with an eastward tilt of the phase surface with height, while above $100 \mathrm{~km}$ the diurnal tide is dominant. The spectrometer also revealed the dominance of the wavenumber-1 (diurnal) component in the equatorial cloud top height. The infrared spectrometer on Venera 15 revealed the meridional structure of the tides in more detail (Zasova et al. 2002, 2007) (Figure 18). At low latitudes, the diurnal component exceeds the semidiurnal one above $90 \mathrm{~km}$, while the semidiurnal component predominates at 58$90 \mathrm{~km}$. At high latitudes, both tides have small amplitudes above $72 \mathrm{~km}$, while below $68 \mathrm{~km}$ the diurnal tide is enhanced and becomes predominant. An eastward tilt of the phase with height is evident in low latitudes. All of the wavenumber-1 (diurnal). 2 (semidiurnal), 3 and 4 components reach maxima in the upper cloud region where the deposition of solar energy is maximized. In the cloud-top height field in the infrared, the diurnal and semidiurnal components are present at low latitudes, while the diurnal component is dominant at high latitudes (Figure 19). Venus Express radio occultations showed that the temperature structure around $64 \mathrm{~km}$ altitude in latitudes $75^{\circ}-85^{\circ} \mathrm{S}$ is dominated by the semidiurnal component (Tellmann et al. 2009). In the region of and above the cloud top (roughly 65 to $90 \mathrm{~km}$ altitude), analyses of the nightside temperature fields retrieved from Venus Express/VIRTIS-M (Grassi et al. 2010, 2014) and VIRTIS-H (Migliorini et al. 2012) measurements also show evidence for thermal tides. 

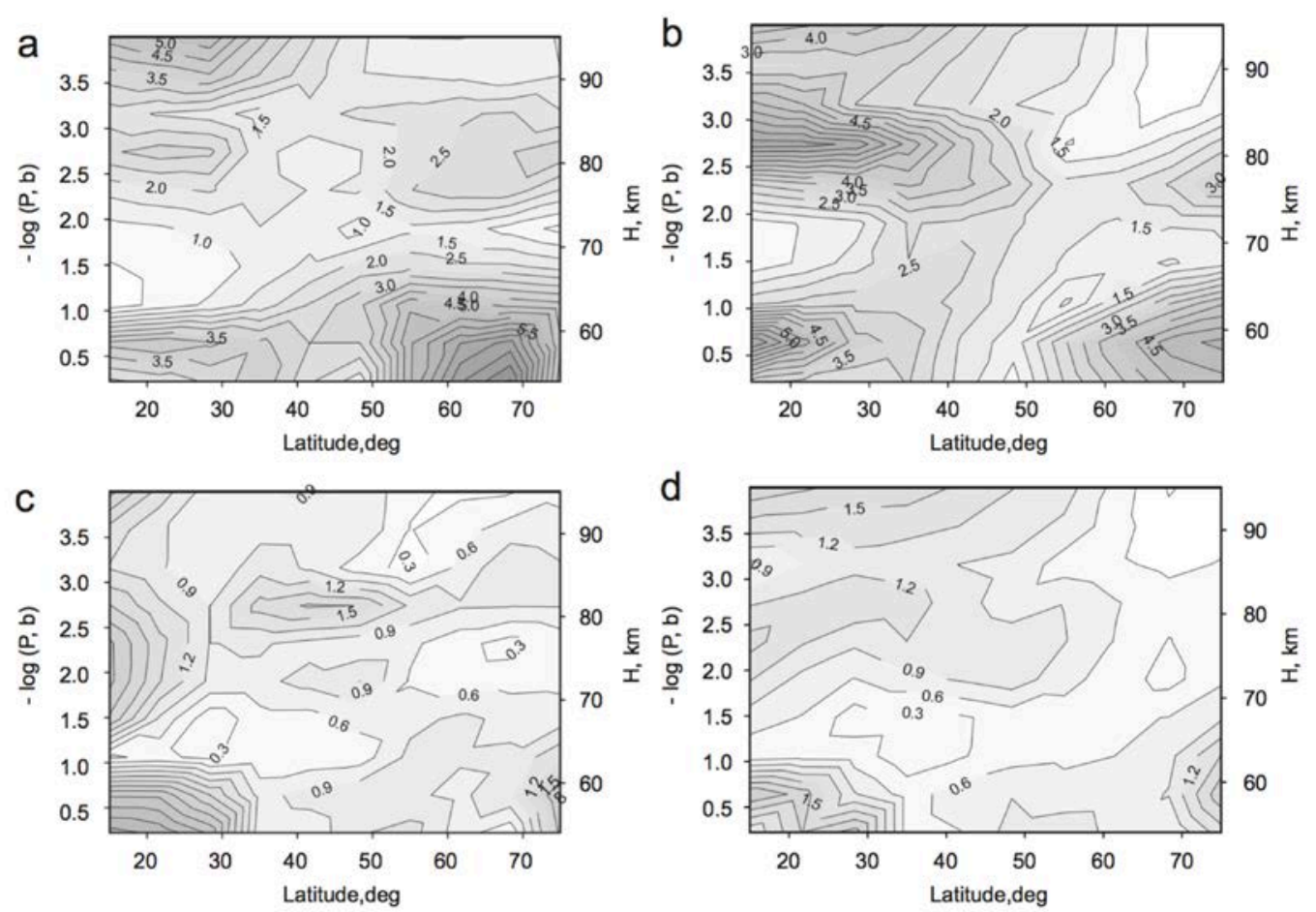

Figure 18. Meridional distribution of the temperature amplitude for the tidal components with 1233 zonal wavenumbers of (a) 1, (b) 2, (c) 3, and (d) 4 obtained from the Venera-15 infrared 1234 1235 spectrometry data (figure after Zasova et al. 2002).

1236 


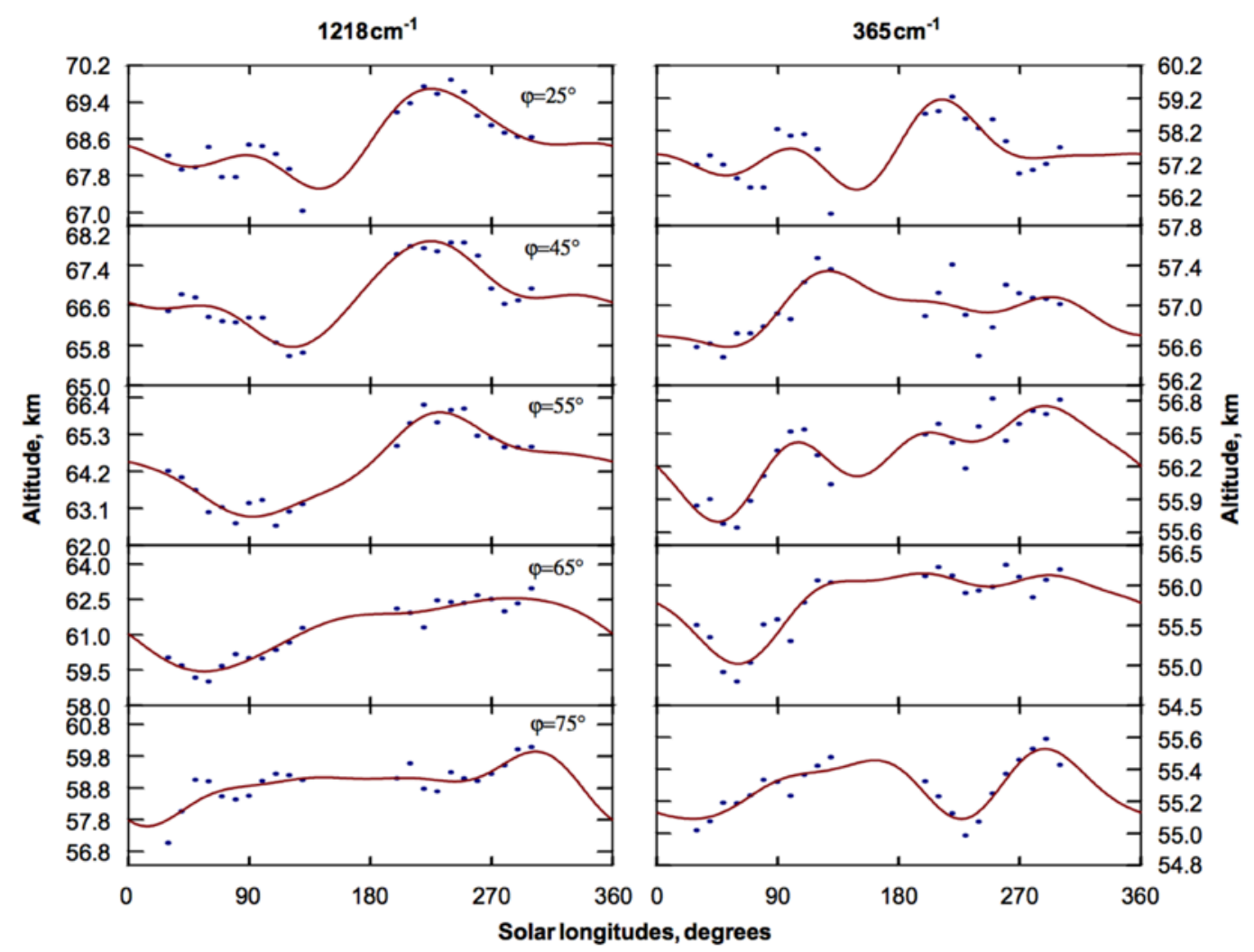

Figure 19. Cloud top heights at different latitudes plotted against the solar longitude, together with fitting curves, obtained from the Venera-15 infrared spectrometry data (figure after Zasova et al. 2002).

The tides were observed also in the wind fields deduced by cloud tracking using ultraviolet images (Limaye 1988) (Figure 20). In low latitudes, the zonal wind is slowest around 10:40 local solar time, and the maximum poleward flow occurs around noon over a broad range of latitudes, leading to horizontal divergence occurring in equatorial latitudes just past noon and convergence around $30^{\circ}-40^{\circ}$ latitudes. Relatively high reflectivities in the ultraviolet are found in regions of convergence and low reflectivities in regions of divergence. The diurnal and semidiurnal components show similar amplitudes in low latitudes, while at mid-latitudes the diurnal component has a larger amplitude than the semidiurnal one (Limaye 2007). Tidal winds at southern polar latitudes were deduced from cloud tracking using infrared images taken by VIRTIS onboard Venus Express (Peralta et al. 2012), showing that the diurnal component is evident in the meridional wind and that a wavenumber-4 component is apparent in the zonal wind. 

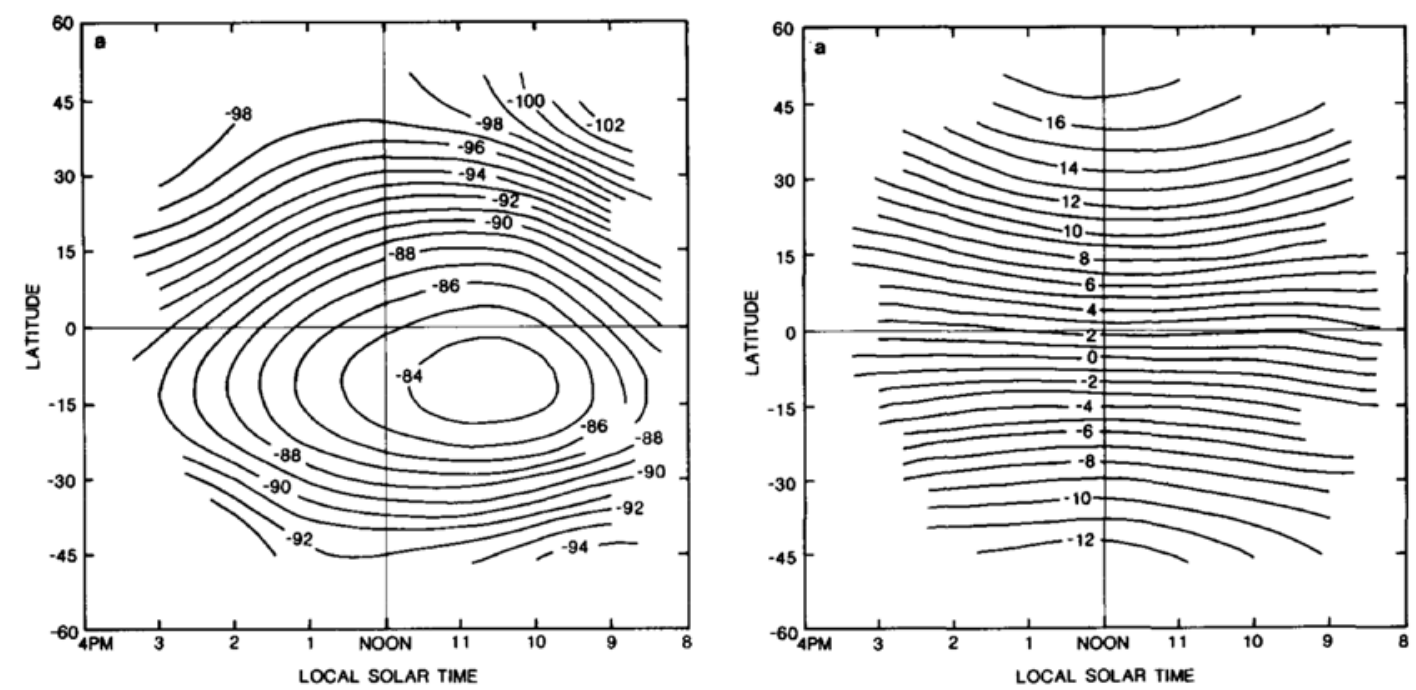

1256

1257

1258

1259

1260

1261

1262

1263

1264

1265

1266

1267

1268

1269

1270

1271

1272

1273

1274

1275

1276

1277

1278

1279

1280

1281

1282

1283

1284

1285

1286

1287

1288

Figure 20. Variations with latitude and solar longitude of (left) the average zonal velocity and (right) the average meridional velocity obtained by cloud tracking using ultraviolet images taken by Pioneer Venus. Units are $\mathrm{m} \mathrm{s}^{-1}$ (figure after Limaye et al. 1988).

A mean poleward flow reaching $10 \mathrm{~m} \mathrm{~s}^{-1}$ was deduced from the cloud-tracked wind field (Limaye et al. 1988; Rossow et al. 1990) and has sometimes been considered as evidence of a strong Hadley circulation driven by solar heating at the cloud level. However, the tidal wind field mentioned above has a strong poleward component on the dayside, and can account for the major part of the mean meridional velocity deduced from dayside observations (Newman and Leovy 1992). On the other hand, Limaye (2007) suggested, based on an extrapolation of a tidal field model fitted to the dayside observations into the nightside, that a strong poleward flow remains on the nightside with a peak near midnight.

Equatorward eddy transport of zonal momentum, which would help sustain the super-rotation, has never been observed in the dayside wind field obtained by cloud tracking (Limaye et al. 1982; Rossow et al. 1990). Limaye (2007) suggested, using tidal field models fitted to the dayside data, that the zonally-averaged eddy momentum transport by the tides can be much different from the estimate from the dayside data alone. The direction of the newly-estimated eddy momentum transport changes between poleward and equatorward, depending upon the observation season, suggesting that it is important to have a full local time coverage for the winds to precisely determine the momentum transport.

\subsection{Models}

Since the pioneering works by Fels and Lindzen (1974) and Ramanathan and Cess (1975), many modeling efforts have been made to understand the observed wave structure and the roles of the tides in the maintenance of the super-rotation. Linear models in spherical geometry show vertical propagation of the diurnal and semidiurnal tides down to the surface and up to $\sim 100 \mathrm{~km}$ altitude (Pechmann and Ingersoll 1984). The models well explain the tidal temperature field observed in the infrared above the clouds. The diurnal temperature amplitude is smaller than the semidiurnal one, even though the diurnal forcing is about twice as great as the semidiurnal one; this feature is 
explained by the difference in vertical wavelength between the diurnal and the semidiurnal tides. The vertical wavelength of the diurnal tide ( $\sim 7 \mathrm{~km}$ near the forcing region) is shorter than that of the semidiurnal tide $(\sim 30 \mathrm{~km})$ because of the difference in the ratio of the zonal wavenumber to the meridional wavenumber (Pechmann and Ingersoll, 1984). Since the thermal forcing is spread out over $\sim 10 \mathrm{~km}$ (Tomasko et al. 1980), i.e., more than the vertical wavelength of the diurnal tide, destructive interference takes place between waves excited at different levels, thereby diminishing the diurnal response. The wavelength of the semidiurnal tide is much longer, so the atmosphere responds more strongly. The linear model by Takagi and Matsuda (2006) also reproduces these features (Figure 21). The terrestrial diurnal tide is weaker than the semidiurnal one for the same reason (Andrews et al. 1987).
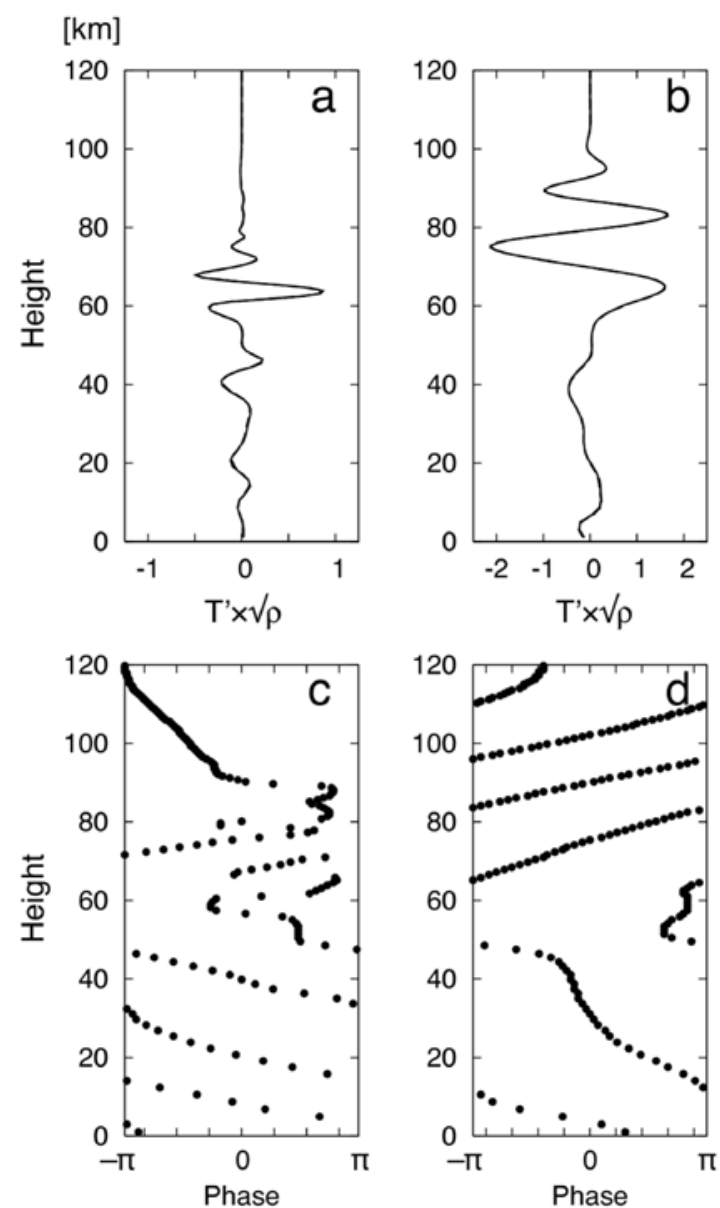

Figure 21. Vertical distributions of the temperature deviation and the phase associated with the diurnal tide $(a, c)$ and the semidiurnal tide $(b, d)$ at the subsolar point. The temperature deviation in unit of $K$ was multiplied by the square root of the basic state density in unit of $\mathrm{kg}$ $\mathrm{m}^{-3}$. (figure after Takagi and Matsuda 2006)

Newman and Leovy (1992) studied the interaction between the zonal-mean field and the wavenumber-1 and 2 components using a semi-spectral model, and showed that the daytime meridional wind is strongly poleward and has a maximum exceeding $10 \mathrm{~m} \mathrm{~s}^{-1}$ in early afternoon due to the tides, giving an explanation for the observed strong poleward flow deduced from 
1311 cloud tracking (Rossow et al. 1990). The model predicts an equatorward flow on the nightside. 1312 Such a model prediction was partly confirmed by observations: Peralta et al. (2012) measured 1313 winds in the Southern polar region using cloud tracking on infrared images and showed that 1314 diurnal tides accelerate the atmosphere poleward on the dayside and equatorward on the nightside. The model also suggests that the superposition of the tidal winds on the zonal-mean winds explains the observed weak mid-latitude jets on the dayside as compared to the zonalmean wind field deduced from the temperature field (Newman et al. 1984).

The excitation of the tides that have prograde momentum at cloud heights and the subsequent vertical propagation lead to removal of prograde momentum from the cloud-level atmosphere, thereby accelerating the cloud-level atmosphere in the retrograde sense (Fels and Lindzen 1974). This acceleration is expected to help maintaining the super-rotation. The tides are thought to be dissipated mostly through radiative damping above and below clouds, leading to deceleration of the super-rotational winds there. Baker and Leovy (1987) developed a three-dimensional semispectral model to study the interaction between the semidiurnal tide and the thermallydriven Hadley circulation around the cloud level, and showed that the tide accelerates the equatorial zonal wind near the cloud-top level and decelerates it at higher levels. In the semispectral model of Newman and Leovy (1992) covering the middle atmosphere, the diurnal and semidiurnal tides were excited, and the equatorial super-rotation was reproduced primarily by the convergence of vertical momentum fluxes due to the semidiurnal tide. It was also suggested that the diurnal tide contributes to the equatorial acceleration by transporting retrograde momentum horizontally from the mid-latitude jet region toward lower latitudes. Such vertical and horizontal momentum transports by the tides were reproduced in Venus GCMs and shown to play crucial roles in the maintenance of the super-rotation at cloud heights (Lebonnois et al. 2010). The decrease of the zonal wind speed with height above the cloud tops (Newman et al. 1984) is attributed at least in part to the vertical momentum transport by the tides (Baker and Leovy 1987; Newman and Leovy 1992).

The model studies mentioned above primarily focused on the vertical propagation of the tides from the cloud level to nearby altitude regions. The resultant momentum transport can explain the observed negative vertical shear above the clouds and the positive shear just below the clouds. However, the maintenance of the deep vertical shear extending from the ground surface to the cloud level needs momentum pumping from the surface; whether the tides can contribute to such deep-atmosphere momentum transport is unclear. Takagi and Matsuda (2005, 2006, 2007) suggested a possibility that the tides reach the surface with sufficient amplitudes to accomplish momentum exchange between the atmosphere and the solid planet (Figure 21). Takagi and Matsuda (2006) examined the detailed structure of the acceleration caused by the tides in the lower atmosphere using a linear model, and showed that the mean zonal flow near the surface is accelerated in the prograde sense (i.e. in the direction opposite to the Venus rotation) by the semidiurnal tide. The zonal flow induced by the semidiurnal tide should be damped by surface friction, and net retrograde angular momentum is then transported from the solid planet to the atmosphere. Takagi and Matsuda (2007) examined this scenario using a nonlinear dynamical model and reproduced the deep vertical shear extending from the surface to the cloud level. We should note, however, that their model excludes the zonal-mean component of the solar heating, and thus a thermally-direct meridional circulation is not generated. The momentum pumping from the surface by the tides is not obtained in the Venus GCM simulations by Lebonnois et al. (2010). 


\section{Instability}

Although solar forcing can account for the formation of the wave-like tides observed around and above the main cloud decks, many other types of waves and eddies observed over a wide range of space and time scales are not directly produced by solar heating and must owe their origin to other mechanisms. Direct instability of the basic atmospheric state on Venus that is produced by differential solar heating may lead to the development of many such waves and eddies. As on the Earth, a variety of mechanisms are likely at work on Venus, ranging from small-scale convection and shear-induced turbulence to large-scale dynamical instabilities that interact with the background rotation of the planet and its atmosphere. The precise mechanism at work, however, depends on the detailed configurations of static stability and wind shear, as well as the boundary conditions. The observational manifestations also depend on the nonlinear development of each instability as it matures and evolves to large amplitudes. These aspects are considered in turn in the following section.

\subsection{Static stability profiles and the Richardson number}

The vertical temperature profiles obtained at different places and local times by the probes and by radio-occultation refractivity measurements (see full details in Chapter 4), have been used to derive the vertical profiles of the atmospheric static stability $S(z)$ and the Brunt-Väisälä frequency $N_{B}(\mathrm{z})$, given by

$S(z)=\frac{d \bar{T}(z)}{d z}+\frac{g}{C_{p}}$

$N_{B}^{2}(z)=\frac{g}{\bar{T}(z)} S(z)$

1385

1386

1387

1388

1389

1390

1391

1392

1393

1394

1395

1396

1397

1398

These two parameters are diagnostic of the stability of the atmosphere to vertical motions. For $N_{B}{ }^{2}(\mathrm{z})<0$ the atmosphere becomes unstable and convection develops. A significant result obtained for Venus by the plethora of spacecraft and instruments sensing the temperature field is that there is a permanent layer at cloud level, between $z \sim 50$ and $60 \mathrm{~km}$, where $S(z) \sim 0$ or $<0$ (Seiff, 1983 and references therein; Young et al., 1987; Jenkins et al., 1994; Tellmann et al., 2009) (Figure 22). Convection and turbulence, with ascending and descending motions, occur in this unstable layer, as noted by the Vega balloons (section 2, Figure 6). Imamura et al. (2014) have shown that this convective layer is due to heating of the cloud base from the deep atmosphere and cooling of the middle cloud to space. They also showed that convection is less active at the subsolar point (compared to high latitudes), because solar energy deposition acts against the destabilizing temperature profile. Above $\sim 60 \mathrm{~km}$ stable conditions $(S(z)>0)$ are found. However, convective cloud structures at $65-70 \mathrm{~km}$ altitudes are observed at the subsolar point (Schubert, 1983; Titov et al., 2012) (Figure 23), a fact that remains to be explained. The 
probe data suggest that there are also other deeper layers that are unstable or close to instability
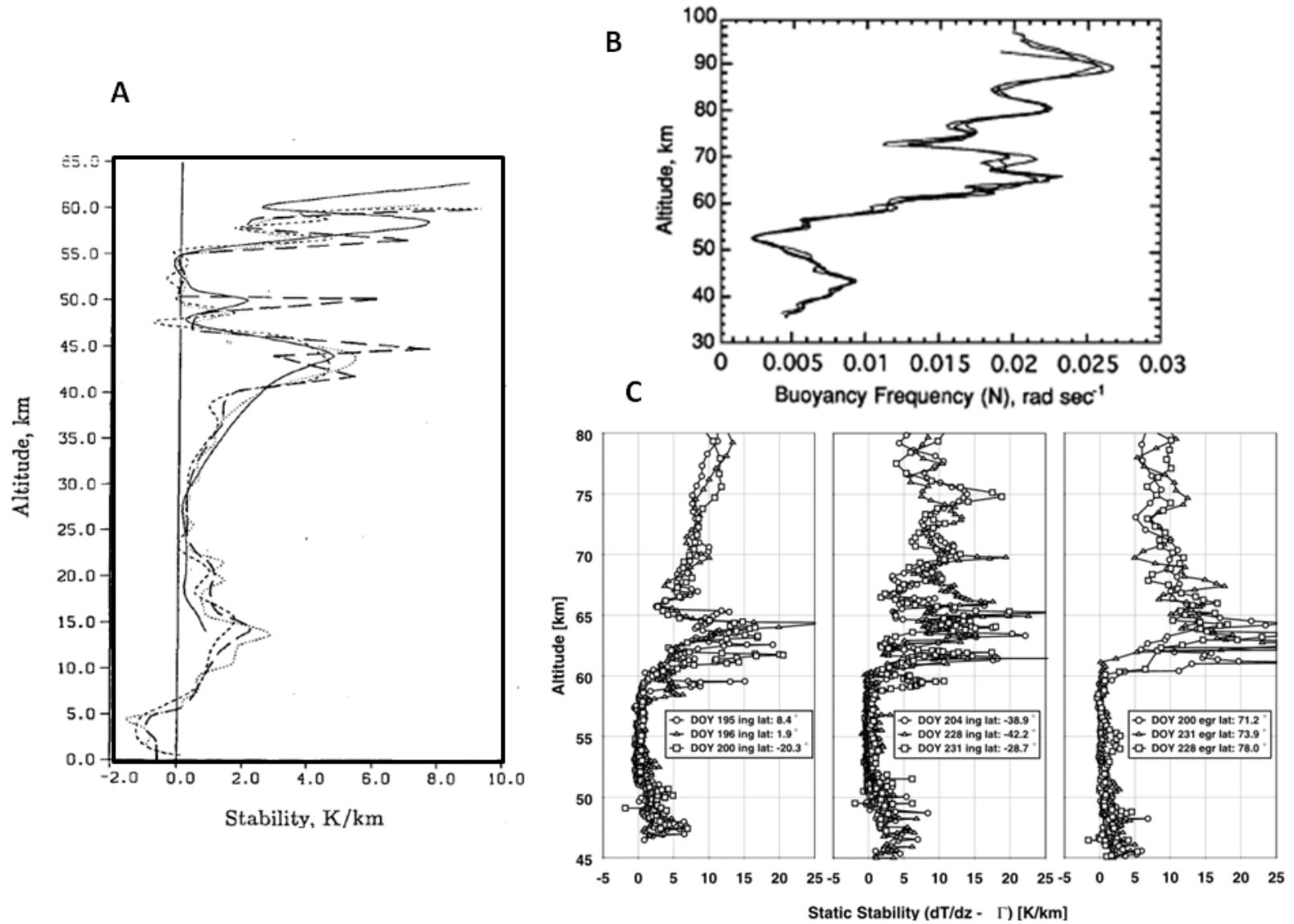

1403

1404

1405

1406

1407

1408

1409

1410

1411

1412

1413

1414

1415

1416

1417

1418

Figure 22. Vertical profiles of the static stability: (A) Static stability measurements from Pioneer-Venus and Vega probes (from Young et al., 1987); (B) Brunt-Väisälä frequency profile from Magellan measurements (Hinson and Jenkins, 1987); (C) Static stability profiles at a variety of latitudes from VERA instrument onboard Venus Express (Tellmann et al., 2009).

A measure of the role played in the vertical instability by thermally produced turbulence and that generated by the vertical shear of the wind is given by the Richardson number, defined a

$$
R i=\frac{N_{B}{ }^{2}}{(\partial u / \partial z)^{2}}
$$

For $R i<-2$, free convection develops and motions are dominated by small scale turbulence (horizontal scale of convective cells $\sim$ vertical scale of the unstable layer, see e.g. Stone, 1976). For $-2<R i<0.25$, forced convection dominates and, as $R i$ increases, larger-scale structures tend to form as supercells. These numbers are somewhat arbitrary for Venus and are based on the theory for terrestrial boundary layer. 


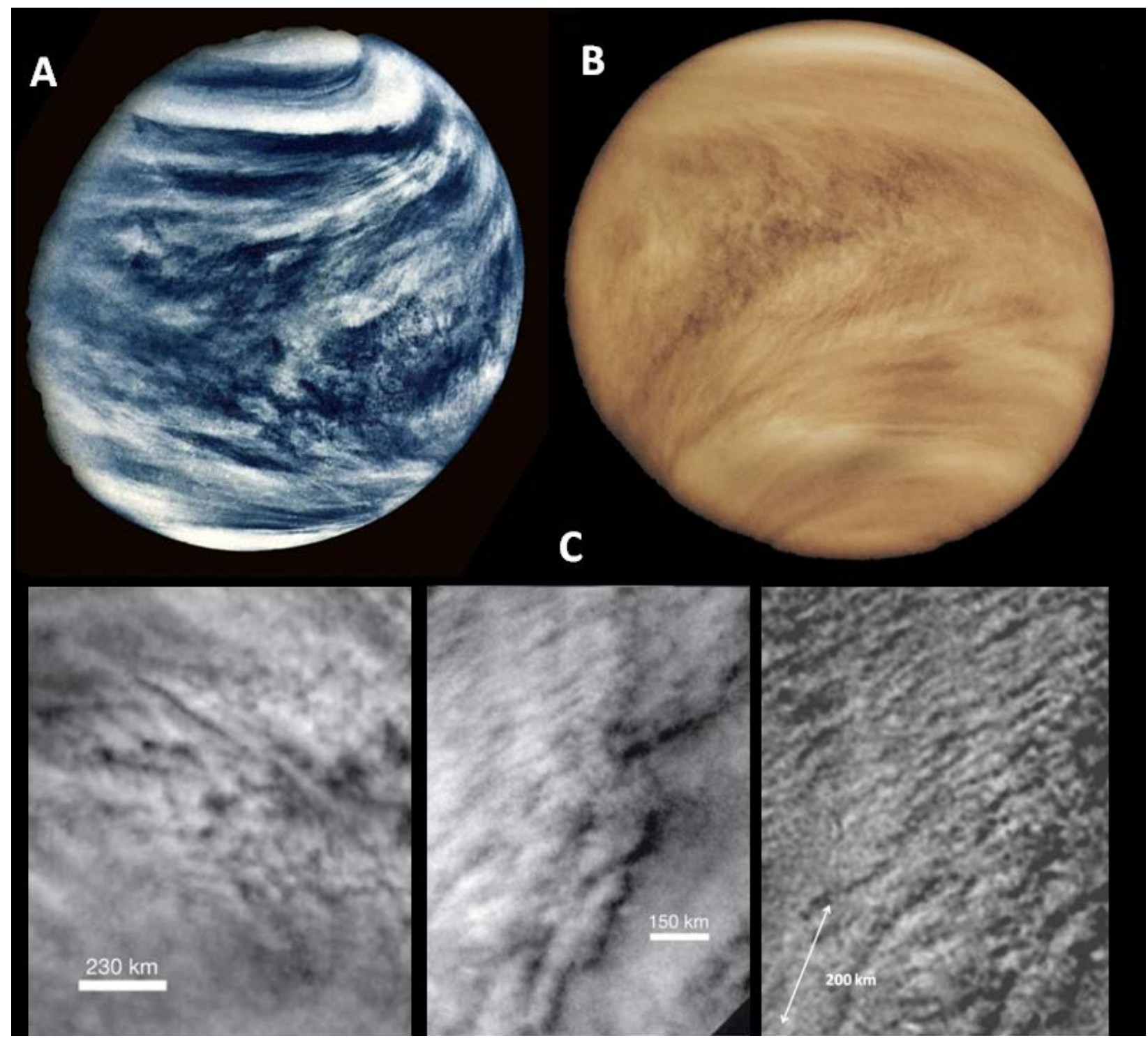

Figure 23. UV images showing turbulent and convective features in the equatorial region of Pioneer-Venus, 5 February 1979 (NASA); (C) Convective features at high resolution from VMC on Venus Express, 22 July 2007 (ESA). The lower right image is a highly processed image to show features at a scale of $25 \mathrm{~km}$.

From the $S(\mathrm{z})$ profiles in figure 22 and the vertical profiles of the zonal wind that can be read thin layers where $S \sim-1 \mathrm{~K} / \mathrm{km}$ and $N_{B}^{2}<0$ ) and $d u / d z=1-8 \times 10^{-3} \mathrm{~s}^{-1}$, which puts Ri typically in the range -1 to 30 , favoring cell and supercell formation at the top of the layer. Piccialli et al. (2012) calculated the averaged Richardson number in a latitude-altitude map and found for this unstable layer $R i \sim$ between 1 and 10 , extending from latitudes $-20^{\circ}$ to $-85^{\circ}$. 
According to the whole long-term 40 years of available data on temperature and winds (19742014), we can conclude that the unstable layer at cloud level is a permanent feature of the Venus atmosphere and extends from pole to pole, playing an important role in Venus's atmospheric dynamics.

\subsection{Convection}

Convection at cloud level is thermally driven from a combination of solar heat deposition and thermal infrared radiation coming upwards from Venus's dense and hot lower atmosphere. Although saturation of $\mathrm{H}_{2} \mathrm{SO}_{4}$ and liquid droplets form clouds, the released latent heating is expected to be small and convection in the cloud layer can be treated as dry. Numerical experiments show that convective plumes develop in the unstable layer, penetrating into the stable layer above it and generating the gravity waves we observe (Baker et al., 1998, 2000; Imamura et al., 2014). From a simple one-dimensional parcel model, the vertical acceleration of 1451 the ascending hot bubbles is given by (Sanchez-Lavega, 2011),

$$
\frac{d w^{\prime}}{d t}=\underbrace{g\left(\frac{T^{\prime}-T}{T}\right)}_{\text {Buoyancy force }}+\underbrace{g \frac{\partial \pi_{D}}{\partial P}}_{\substack{\text { Dynamic } \\ \text { Pressure }}}-\underbrace{C_{D} \frac{w^{2}}{r_{0}}}_{\text {Friction }}
$$

1453

1454

1455

1456

1457

1458

1459

1460

1461

1462

1463

1464 1465 1466

where the velocity is $w^{\prime}$ and $T^{\prime}$ and $T$ are the temperatures of the parcel and surroundings respectively, and as indicated, should be considered as an upper limit. Here $\pi_{D}$ is the dynamic pressure, $C_{D}$ is a drag coefficient, and $r_{0}$ is a characteristic parcel size. The buoyancy contribution is the most important term, giving velocities

$$
\left.w_{\max } \approx \sqrt{2 g\left(\frac{\Delta T}{T}\right) \Delta z} \sim 15-20 \mathrm{~ms}^{-1} \text { (for } \Delta T \sim 1-2 \mathrm{~K}, T \sim 350 \mathrm{~K}, \Delta z \sim 5 \mathrm{~km}\right)
$$

Alternatively, application of mixing-length theory for free convection (Priestly, 1959; Gierasch et al., 1997) predicts lower vertical velocities given by

$w_{m \ell t} \approx\left[\frac{R_{g}}{C_{p}} \frac{F_{Q}}{\rho}\right]^{1 / 3} \sim 1 \mathrm{~ms}^{-1}$

for $R_{g} / C_{p} \sim 0.22$, being $C_{p}$ the specific heat at constant pressure, $R_{g}$ is the specific gas constant $F_{Q}$ $\sim 5-15 \mathrm{Wm}^{-2}$ is the convective heat flux at the base of the convective layer (Imamura et al., 2014) and $\rho \sim 1.5 \mathrm{~kg} \mathrm{~m}^{-3}(P=1 \mathrm{bar}, T=347 \mathrm{~K}$ at $z=50 \mathrm{~km})$. These values comprise those measured with the Vega 1 and 2 balloons (Figure 6). Additionally, as shown in Figure 23, the highest resolution and well contrasted images show features with round, cell-like shapes and a horizontal scale $\sim 25-50 \mathrm{~km}$ that suggest its convective origin. All this supports dry convection as a mechanism at work in this unstable layer. According to Figure 22A, convective motions could be 
expected also to occur close to the surface and in a layer between $\sim 24-28 \mathrm{~km}$ above the surface where $S \leq 0$.

\subsection{Barotropic, Baroclinic and shear instabilities}

Although Kelvin-Helmholtz instabilities and free convection may lead to the development of small-scale eddies in the Venus atmosphere, it is likely that the large-scale wave structures seen in UV images and in fields of wind and temperature are produced by other processes. At least three types of instability process may lead to the generation of the types of large-scale waves and eddies discussed above in Section 4. Barotropic and baroclinic instabilities may be capable of directly energizing zonally-propagating Rossby and equatorial planetary waves, and have been studied for Venus in models at various levels of complexity. Close to the equator, many of the conditions necessary for inertial instability are also satisfied, although direct observational evidence for this instability is somewhat elusive.

\subsubsection{Large-scale barotropic instability}

Barotropic instability was the first kind of dynamical instability process to be invoked as the origin for large-scale waves in Venus's atmosphere that might also transport angular momentum equatorwards (e.g. Elson 1978, Rossow \& Williams 1979, Elson 1982, Michaelangeli et al. 1987). It is a horizontal shear instability of the Rayleigh (inflection-point) variety, for which a necessary (but not sufficient) condition for its occurrence (e.g. see Holton 2004, Andrews et al 1987, Vallis 2006) is for the meridional gradient of absolute or potential vorticity (or its equivalent in spherical geometry) to change sign somewhere within the horizontal domain of interest (also known as the Rayleigh-Kuo stability criterion; e.g. Vallis 2006). For zonal flows that fulfill this necessary condition and are actually unstable, the typical result is a growing, wave-like disturbance that propagates zonally, effectively energizing a barotropic Rossby wave in mid-latitudes. The essential mechanism can be viewed as resulting from the mutual resonant interaction between two zonally-propagating Rossby waves, supported by two horizontally adjacent regions of meridional potential vorticity gradient $\partial \overline{\mathrm{q}} / \partial \mathrm{y}$ of opposite sign (e.g. see Lindzen 1988, Vallis 2006), leading to a meridional flux of zonal momentum $\overline{u^{\prime} v^{\prime}}$ in the same sense as $\partial \bar{u} / \partial y$. Here u' and v' are the zonal and meridional eddy velocity components. This requires the resulting waves to "lean into" the shear of the background zonal flow so as to increase the kinetic energy of the waves at the direct expense of the zonal flow.

The most likely location for barotropic instability on Venus is in the vicinity of the main cloud decks, where the zonal flow is strongest and where strong lateral shears are found close to the edge of the polar vortex in each hemisphere. This was originally considered by Limaye \& Suomi (1977) and Travis (1978) in the context of winds derived from tracking features in UV images from Mariner 10, and analysed theoretically by Travis (1978) and Elson (1978). Travis (1978) and Elson (1978) used a linearized barotropic semi-analytical model to investigate the stability of a set of zonal wind profiles that were idealizations of the measured profiles from Mariner 10 and found that profiles with mid-latitude jet-like structures could be barotropically unstable to wavelike disturbances with maximum growth rates at zonal wavenumbers ranging from $\sim 1-3$ to 7 . The 
most unstable modes, however, were largely concentrated at middle latitudes. These calculations were later extended to more realistic cloud-level wind profiles up to the poles, based on further measurements from Pioneer Venus, by Elson (1982) and Michaelangeli et al. (1987), also using a linearized barotropic numerical model. Their results are discussed in the context of the polar vortices presented in section 7 .

\subsubsection{Baroclinic instability}

The possible occurrence of baroclinic instability has also been discussed in various forms on Venus (Young et al, 1984, Takagi \& Matsuda 2005b, Takagi \& Matsuda 2006b, Sugimoto et al. 2014a). Baroclinic instability may occur in the presence of stable stratification and a horizontal thermal gradient associated with a vertically-sheared, balanced zonal flow. The growing disturbances gain energy primarily at the expense of the available potential energy of the background zonal flow by transporting heat along trajectories projected onto the meridional plane that are inclined at an angle intermediate between those of the geopotentials and surfaces of constant potential temperature (e.g. Holton 2004, Vallis 2006). A necessary condition for baroclinic instability, analogous to the Rayleigh-Kuo condition for barotropic instability, is that the horizontal gradient of potential vorticity, $\partial \bar{q} / \partial y$, must change sign in the vertical direction within the domain (e.g. Holton 2004, Vallis 2006).

The very low rotation rate of Venus itself, however, would seem to make this mechanism unlikely to occur in the presence of a relatively weak zonal flow whose absolute rotation rate and vorticity was comparable to or smaller than that of the underlying planet. This is because the first baroclinic Rossby deformation radius, $N_{B} H / f$, is then much larger than the radius of the planet. Here $H$ is the scale-height (Sánchez-Lavega, 2011). But at levels close to the visible clouds, the local rotation rate is much faster (period around 4 Earth days) and may be more conducive to baroclinic instability. These conditions were satisfied close to the main cloud decks in the idealized basic states investigated by Young et al (1984) in their linearized model study, including the reversal of the sign of $\partial \overline{\mathrm{q}} / \partial \mathrm{y}$ in the vertical, in association with vertical changes in the static stability and curvature of the zonal flow at these altitudes. Young et al. (1984) analysed the linear stability of such baroclinic basic states in flows confined to an altitude range between 25 and $70 \mathrm{~km}$, within which they assumed a value of Coriolis parameter, $f$, consistent with the mean rotation rate of the cloud level winds. They identified a range of growing instabilities at zonal wavenumbers $n=1-8$, including low wavenumber $(n=2)$ modes centered close to the pole, much as found above arising from barotropic instabilities. These modes were also confined in the vertical, however, mostly between around 45 and $60 \mathrm{~km}$ with westward vertical phase tilts typical of growing baroclinic waves. The most rapidly growing modes, however, tended to be of relatively high zonal wavenumber $(\sim n=7)$ and centred at low or middle latitudes, although this was dependent upon the detailed structure of the basic zonal state.

These solutions also included modes with secondary maxima on the equator itself. These were interpreted by Young et al. (1984) as equatorially trapped Kelvin waves that were being coherently excited by a baroclinically unstable (Rossby-like) wave centred around $20^{\circ}$ latitude. This is effectively a generalization of the resonant Rossby-Rossby wave barotropic instability mechanism to include the resonant interaction of an equatorial Kelvin mode with a 
1563 (baroclinically unstable) Rossby wave. This is similar in many respects to the mechanism 1564 identified in the shallow water model of Iga \& Matsuda (2005) that led to a growing equatorial 1565 Kelvin mode through resonant interactions with nearby Rossby, inertia-gravity or continuous 1566 spectrum modes associated with the presence of critical layers at low latitudes - similar to the 1567 Rossby-gravity, Rossby-Kelvin or Yanai-Kelvin instabilities discussed e.g. by Sakai (1989), 1568 Gula et al. (2009) and Ribstein et al. (2014). Similar Kelvin-like waves were also found to 1569 emerge in the presence of cloud radiative feedbacks in the model investigated by Smith et al. 1570 (1993), offering another possible mechanism for exciting these features. Resonant Kelvin wave instabilities were also noted during the spin-up of slowly-rotating model simulations by Mitchell \& Vallis (2010), though such Kelvin-like modes tended to dissipate and decay later in the simulation, becoming negligible as the flow equilibrated.

Further idealized studies of linearized baroclinic instability in cyclostrophic flows were carried out by Takagi \& Matsuda (2005b, 2006b), without making the pseudo-geostrophic approximation used by Young et al. (2004) that entailed shifting to a reference frame in which the basic state was effectively geostrophic within the main cloud deck. Their results largely confirmed those of Young et al. (1984) where the effective Rossby number was sufficiently small, but at much larger (non-geostrophic) Rossby numbers, the character of the instability changed to favour lower zonal wavenumber modes centerd in the deep atmosphere, below the main cloud decks, at mid-latitudes. The modal structures were also quite complicated with significant meridional phase tilts and a corresponding momentum flux directed towards the equator.

The fully nonlinear development of baroclinic instabilities under Venusian conditions has been considered implicitly within a number of global circulation model studies of the Venus atmospheric circulation (see Section 8), though few of these have so far investigated this process in any detail. Sugimoto et al. (2014), however, have used a full primitive equation GCM with realistic planetary parameters for Venus to examine the nonlinear development of some idealized baroclinically unstable flows. These included a pure baroclinic state with a linear verticallysheared zonal flow in local solid-body rotation at each altitude, as well as more realistic flows with mid-latitude jets. Figure 24 shows some example fields from their pure baroclinic simulation. Like the basic states considered by Young et al. (1984), the horizontal potential vorticity gradient (Figure 24(a)) reversed sign with height within the main cloud layers, mostly associated with strong changes in static stability within the cloudy layers and concentrated at mid-to-high latitudes. The developing baroclinic waves grew strongly within the layer from 50$65 \mathrm{~km}$ altitude, much as predicted from the linearized model of Young et al. (1984), with the eddies transporting heat from low latitudes polewards and initially feeding angular momentum into weak mid-latitude jets. In the later stages of the flow evolution, the eddies developed a meridional tilt in the sense to transport angular momentum polewards, at least at the level shown in Figure 24(b), though some cases produced equatorward momentum transport at higher altitudes. In the vertical, the growing waves developed a noticeable westward phase tilt with height in the sense expected for "classical" baroclinic instability (e.g. Holton 2004, Vallis 2006; 
(a) Solid-body rotation (Case SO)

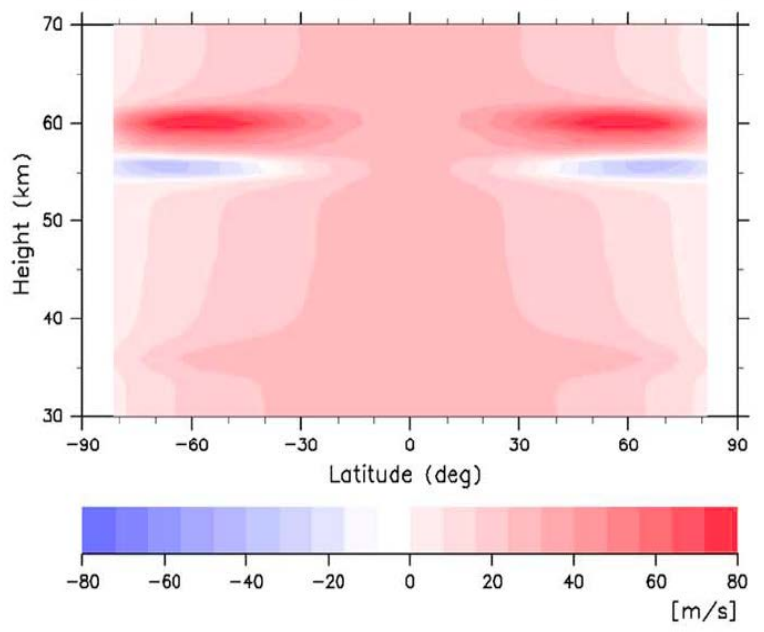

(b) Vorticity, day 360
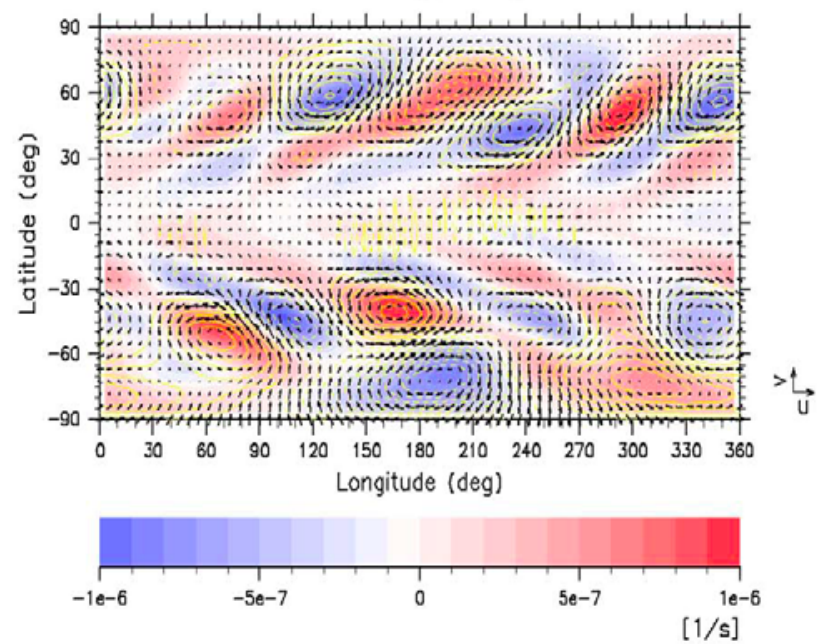

1606

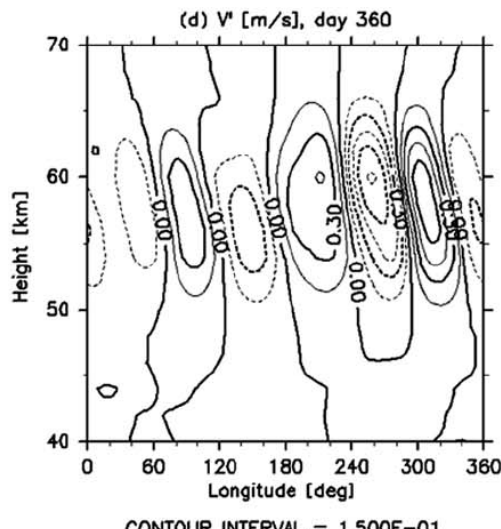

CONTOUR INTERVAL $=1.500 E-01$

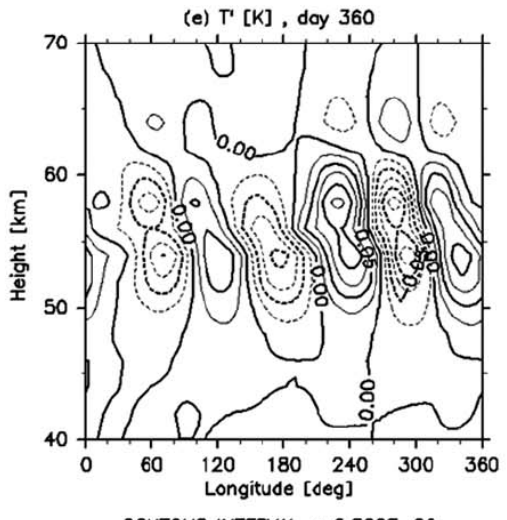

CONTOUR INTERVAL $=2.500 E-02$

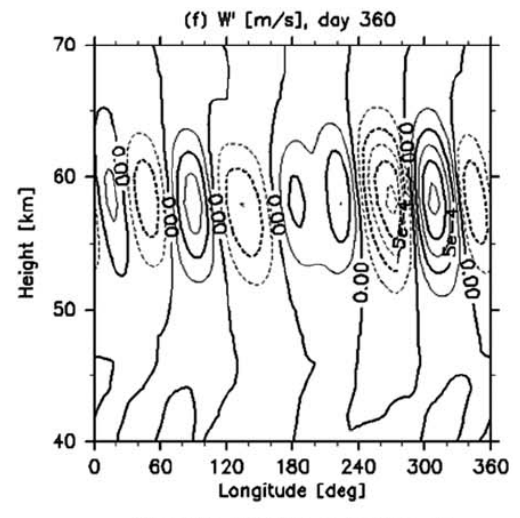

CONTOUR INTERVAL $=2.500 E-04$

Figure 24: Results from numerical simulations (by Sugimoto et al. 2014) of the nonlinear baroclinic instability of a cyclostrophically balanced, vertically-sheared initial state in local solid-body rotation under Venus conditions; (a) basic state potential vorticity gradient, (b) velocity vectors and eddy vorticity field at $54 \mathrm{~km}$ altitude, (c)-(e) longitude-height maps of (c) eddy meridional velocity, (d) temperature perturbation and (e) vertical velocity. (b)-(e) are shown at day 360 from an initialized state.

see Figure 24(c)-(e)). Similar results were also found for more realistic initial flow structures, although when zonal jets were present the eddies developed a more mixed baroclinic-barotropic structure.

The development of instabilities with a mixed barotropic-baroclinic character during their nonlinear life cycle seems to be typical of many flow realizations resembling the observed Venus conditions, although the relative dominance of either type of instability on Venus remains to be established quantitatively. Very few studies so far have sought to quantify the detailed energetic exchanges during the establishment of Venus-like super-rotating circulation patterns. Lorenz 1626 
Venus-like model simulations using different dynamical model cores (cf Section 8.2). Although some differences of detail between different models were evident in their comparison, a common trend was found in which eddies gained energy from the zonally-symmetric flow components via both baroclinic and barotropic energy conversions, with the barotropic term being typically three or more times larger than the baroclinic conversion rate. This suggests that barotropic instabilities are likely to be somewhat more important energetically under Venus conditions, but baroclinic effects are also significant energetically and affect the structure of developing waves, leading e.g. to phase tilts with height.

\subsubsection{Inertial instability}

Another form of instability is found if the product $f q<0$ anywhere within the domain, where $q$ may be either the absolute vorticity or (Ertel) potential vorticity (e.g. see Andrews et al (1987)). This condition was also deduced in the context of Venus's atmosphere for a cyclostrophic regime in the primitive equations using Ertel's potential vorticity by Peralta et al. (2014a). Such a condition, where the absolute or potential vorticity takes the "wrong" sign in a given hemisphere, is most likely to occur close to the equator, for which cross-equatorial transport of vorticity can lead to positive $q$ crossing into the southern hemisphere and vice versa.

The manifestation of this instability is commonly of a zonally symmetric form, with vertically stacked rolls in the meridional plane of relatively short vertical wavelength and small lateral scale (e.g. Hua et al. 1997, Griffiths 2003). This is often to be seen in numerical model simulations, for example, where grid-length perturbations may occur close to the equator in zonal mean fields of temperature and velocity. But other forms may be possible, especially if the instability occurs at the same time as other types of instability so their effects reinforce each other. To date, however, there are few if any clear examples of observed phenomena on Venus that can be unambiguously attributed to inertial instability.

\section{Polar vortices and the cold collars}

While the Venusian circulation at the cloud tops is dominated by zonal retrograde super-rotation up to about $+/-60^{\circ}$ latitude (Schubert 1983; Gierasch et al. 1997; Peralta et al. 2007), at higher latitudes the dynamics of the atmosphere is dominated by polar vortices, regions of low-altitude clouds and high temperatures which sometimes take on the appearance of a dipole surrounded by a cold "collar".

The name polar vortex should be clarified, since it has been applied in different contexts to designate different structures present in the Venus polar atmosphere. In dynamical terms, a vortex is characterized by a roughly circular region of low relative vorticity (the "eye"), surrounded by a narrow annulus of high vorticity gradient (the "eyewall"). The vorticity then decreases slowly, farther away from the center. Strong atmospheric vortices trap air in their cores and isolate it, preventing mixing with air outside the eyewall for long periods of time.

In morphological terms, a vortex is characterized by swirling clouds that move around a common center of rotation and that often take on the appearance of a spiral. In the best studied examples of large-scale atmospheric vortices - terrestrial tropical cyclones - the eyewall is seen in radar reflectivity as a region of intense cloud formation. Downwelling occurs in the center of the eye, 
1673 causing regions devoid of clouds, known as cloud clearings, but with strong upwelling in the eyewall cloud layer. The eye of the vortex, the outer boundary of which is where upwelling is the most intense, is mostly clear of clouds. However, in many cases of strong hurricanes overshooting clouds are observed in this region.

A third, thermal definition of the Venus polar vortex has often been used: that of a region of infrared-bright warm air, localized close to the pole. On Venus, due to the lack of in situ measurements of the winds, both the morphological and the thermal definition have primarily been used to refer to the spiraling, warm structure seen at both polar regions. Here we refer to the infrared-bright core as the polar vortex core.

The first view of swirling polar clouds indicative of a hemispheric vortex structure on Venus was obtained by Mariner 10, on the basis of the appearance of cloud markings in a polar projection of Southern hemisphere UV pictures, and a similar cloud pattern was suggested to exist at the northern hemisphere (Limaye and Suomi 1977; Suomi and Limaye 1978). The northern vortex was also captured in these images (Fig. 12.9 in Taylor, 2014) and in the Galileo flyby images (Peralta et al., 2007).

Infrared Pioneer Venus temperature maps at $11.5 \mu \mathrm{m}$ also revealed an ellipsoidal dipole at the northern polar region (Taylor et al. 1980), and a southern counterpart was later discovered by the Venus Express VIRTIS instrument at $5.0 \mu \mathrm{m}$ (Piccioni et al. 2007) (Figure 25). While at $11.5 \mu \mathrm{m}$ the northern dipole was seen as a 10 degree-wide, 20 degree-long ellipse (Taylor et al. 1980), at 3.9 and $5 \mu \mathrm{m}$ a variety of other morphologies have been seen (Luz et al. 2011; Garate-Lopez et al. 2015). In VEx-VMC UV images, the dipole is seen as a dark oval surrounded with dark streaks (Markiewicz et al. 2007). It is, however, not always visible, being sometimes covered by the UV bright polar haze, a hood-like structure that extends from latitude $55^{\circ}$ to the pole. Interestingly, UV images from PV did not reveal a polar vortex, also perhaps due to a thick haze 1701 at high latitudes (Zasova et al. 2007; Esposito et al. 1997). 


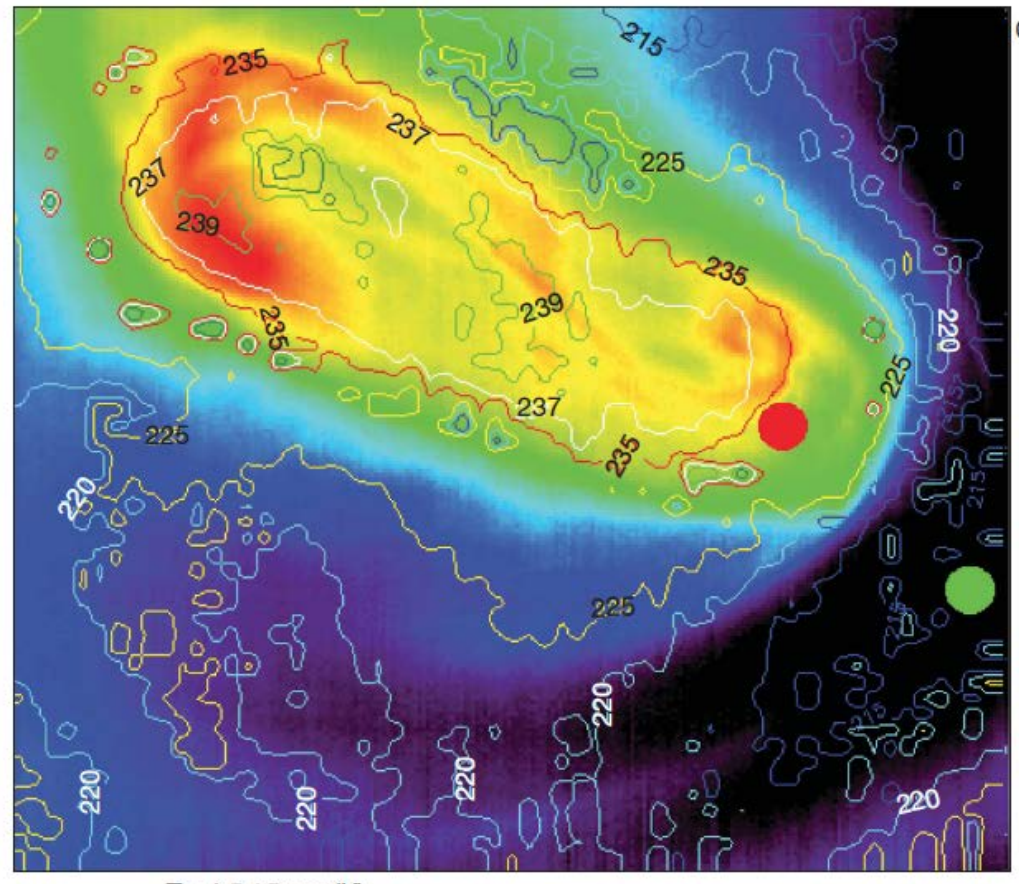

$T_{\mathrm{B}}$ at $5.05 \mu \mathrm{m}(\mathrm{K})$

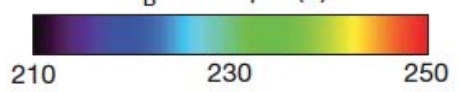

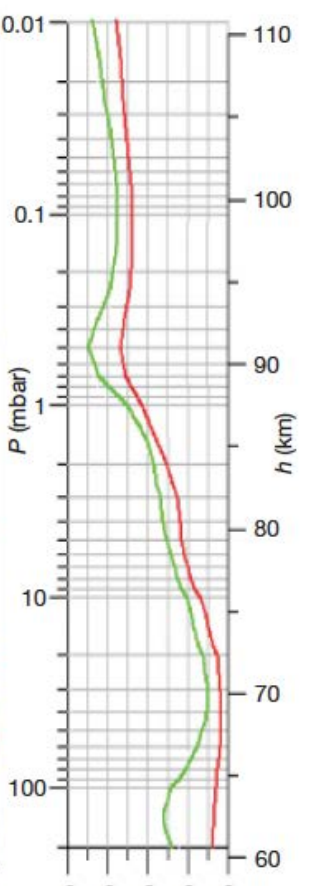

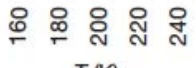

$T(\mathrm{~K})$

Figure 25. The polar vortex under dipole morphology in its highest-thermal-contrast atmospheric layer. Left, brightness temperature, $T_{B}$, at $5.05 \mu \mathrm{m}$ of the dipole and the cold collar region. The contours are the real atmospheric temperatures retrieved from VIRTIS measured radiances, corresponding to a pressure of $200 \mathrm{mbar}$ (about $60 \mathrm{~km}$ ). Right, the atmospheric vertical temperature profile in two different regions. The green curve is retrieved in the green spot on the left image, inside the polar collar. The red curve is from the red spot inside the dipole. (Piccioni et al. 2007)

\subsection{Polar wind field}

There have been only a few in situ measurements of polar winds on Venus, and they have been extensively reviewed elsewhere (Schubert 1983; Gierasch et al. 1997). Other than radio tracking of Venera and Pioneer Venus entry probes (Marov 1978; Schubert 1983), cloud top polar wind measurements have been obtained solely based on tracking cloud features at various wavelengths: in the UV by Mariner 10 (Limaye and Suomi 1981), at $365 \mathrm{~nm}$ by the Pioneer Venus Orbiter OCPP instrument (Rossow, Del Genio, and Eichler 1990), at 418 nm (Toigo, Gierasch, and Smith 1994), $418 \mathrm{~nm}$ and $986 \mathrm{~nm}$ (Peralta et al. 2007) by Galileo SSI, at $365 \mathrm{~nm}$ by Venus Express VMC (Moissl et al. 2009) and at $380 \mathrm{~nm}, 980 \mathrm{~nm}$ and $1.74 \mu \mathrm{m}$ by VIRTIS (Sánchez-Lavega et al. 2008; Hueso et al. 2012). Cloud tracking was also used to characterize gravity waves, based on non-LTE $\mathrm{CO}_{2}$ emissions at $4.3 \mu \mathrm{m}$ (Garcia et al. 2009) and on scattered UV and near-infrared thermal wavelengths (Peralta et al. 2008). Garcia et al. (2009) identified turbulence originating at the polar vortex as the source of gravity waves observed in the $110-$ 
$140 \mathrm{~km}$ altitude range, and the gravity wave activity in number and amplitude was strongest when the vortex was on the dayside, which suggested they were thermally excited.

The pattern of swirling clouds seen in both hemispheres at UV wavelengths, together with the infrared-bright cloud clearings observed at both poles by Pioneer Venus and Venus Express, have been interpreted as the result of the combined poleward and descending branches of a Hadley cell that extends from the equator to both poles (Luz et al., 2011). Mean cloud height measurements appear to support this interpretation (Figure 26). Although the mean cloud top altitude is close to $74 \mathrm{~km}$ from the equator up to mid-latitudes in both hemispheres, in the polar region it decreases by 5-9 $\mathrm{km}$ and can experience fast variations of about $1 \mathrm{~km}$ within hours (Ignatiev et al. 2009). Although lower altitude polar clouds are suggestive of polar downwelling, this interpretation raises the question of meridional wind measurements consistent with a poleward branch of the Hadley cell at latitudes higher than $70^{\circ}$, which are so far inconclusive. Despite measurements suggesting poleward winds at these latitudes (Hueso et al., 2012), their high statistical uncertainties do not allow ruling out equatorward winds. The question thus remains as to whether the Hadley cell extends beyond the cold collar latitudes and what produces the spiraling appearance of the clouds in the polar region.

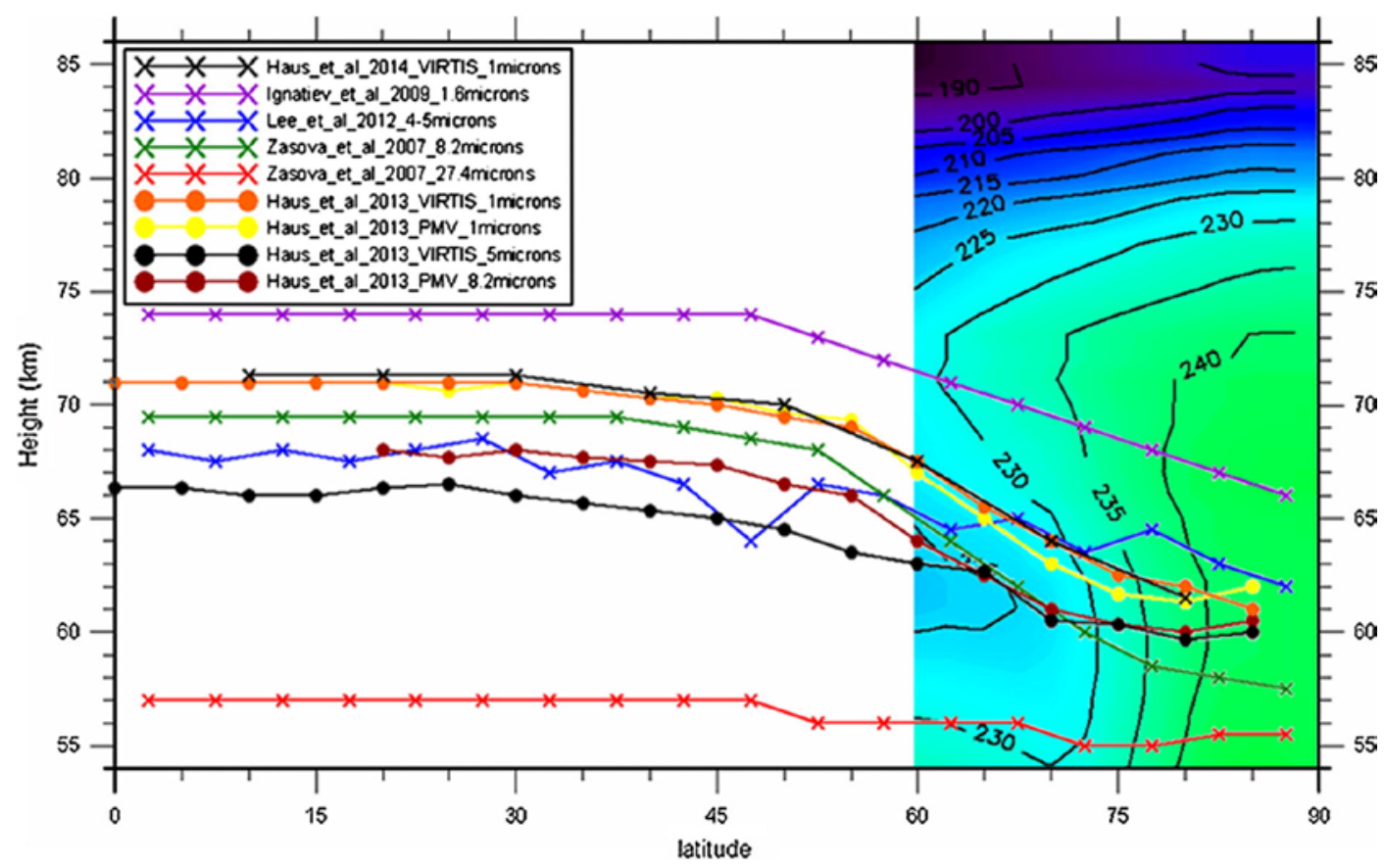

Figure 26 Cloud top altitude (level at which optical depth at the given wavelength is 1) as a function of wavelength from a synthesis of data from Northern and Southern hemispheres. The temperature structure is shown for the Southern Hemisphere (from Garate-Lopez et al., 2015)

Also noteworthy is the fact that the subsolar-to-antisolar flow, measured by Lellouch et al. (2008) at lower latitudes, does not seem to reach the high latitudes. They speculate that this might result from an interaction of the tropospheric Hadley cell with the polar vortex. 
1756

1757

1758

1759

1760

1761

1762

1763

1764

1765

1766

1767

1768

1769

1770

1771

1772

1773

1774

1775

1776

1777

1778

1779

1780

1781

1782

1783

1784

1785

1786

1787

1788

1789

1790

1791

1792

1793

1794

1795

1796

1797

1798

1799

1800

1801
Despite the unfavorable viewing geometry in flyby missions (Mariner 10, Galileo, Cassini and Messenger) and the lower contrast of polar clouds in UV images, a sizeable fraction of wind measurements have been reported for the polar regions of Venus (Limaye 2007; Peralta et al. 2007; Sánchez-Lavega et al. 2008; Moissl et al. 2009; Luz et al. 2011; Hueso et al. 2012), albeit at a relatively poor spatial resolution relative to lower latitudes. Polar winds have been derived from VEX/VIRTIS cloud tracking at UV (380 nm), near infrared $(980 \mathrm{~nm})$ and at four infrared wavelengths $(1.74,2.30,3.93$ and $5.02 \mu \mathrm{m})$. The $380 \mathrm{~nm}$ radiation corresponds to absorption by the cloud tops at altitudes between 62-70 km (Sanchez-Lavega et al., 2008), while at $980 \mathrm{~nm}$, photons are more penetrating and reach the base of the upper cloud within an altitude range 58 $64 \mathrm{~km}$ (Peralta et al., 2017). The wavelengths of 1.74 and $2.30 \mu \mathrm{m}$ are mainly used to probe the night side (where thermal radiation from the lower atmosphere is dominant), since they are overexposed in the dayside due to the contribution of solar radiation scattered from the upper cloud. At $5 \mu \mathrm{m}$ this solar radiation component is negligible relative to the thermal emission from the upper cloud, allowing both day and night side studies, and at $3.93 \mu \mathrm{m}$ the scattered radiation is also negligible except at low sun-zenith angles (Peralta et al. 2012).

The first latitudinal zonal wind profile at cloud tops altitude since Pioneer Venus has been obtained by Sanchez-Lavega et al. (2008). At high latitudes, they measure zonal wind speeds that decrease roughly with latitude (from $40+/-15 \mathrm{~m} \mathrm{~s}^{-1}$ at $70^{\circ} \mathrm{S}$ to nearly zero at the pole) with no detectable vertical wind shear (values lower than $15 \mathrm{~m} \mathrm{~s}^{-1}$ ) between the altitudes of the $1.74 \mu \mathrm{m}$ and UV filters (47 and $66 \mathrm{~km}$, respectively), indicating the possibility of a vertically coherent vortex structure. The wind profile was nearly flat in the range $80^{\circ} \mathrm{S}-70^{\circ} \mathrm{S}$. Further measurements by Moissl et al. (2009), in the UV, and by Luz et al. (2011) in the $70^{\circ} \mathrm{S}-90^{\circ} \mathrm{S}$ range at $5.0 \mu \mathrm{m}$ confirmed this behavior within their error bars, but measured winds differed by up to $10 \mathrm{~m} \mathrm{~s}^{-1}$.

\subsection{The cold collar}

The cold collar, also known as the circumpolar collar, is a region of colder air located at latitudes between $60^{\circ}$ and $80^{\circ}$ at both poles, at a radial distance of about $2500 \mathrm{~km}$. The cold collar manifests in the UV albedo as bright and uniform because of its positive lapse rate, suppresses vertical mixing and cuts off the supply of ultraviolet absorbers from below (Titov et al. 2008). It was first detected as a wavenumber $n=1$ feature in the temperature distribution in Pioneer Venus Orbiter Infrared Radiometer (OIR) observations (Taylor et al., 1979a, cited in Schubert, 1980), but VEX/VeRa observations of the southern collar have since identified a wavenumber $n$ $=2$ structure at approximately $62 \mathrm{~km}$. At the latitudes of the cold collars, the tropopause altitude is higher by $\sim 2-3 \mathrm{~km}$ relatively to surrounding latitudes, while the temperature drops roughly 60 $\mathrm{K}$ (Tellmann et al. 2009). The cold collar divides the middle atmosphere vertically: below it the atmosphere cools with increasing latitude, while above, it warms with increasing latitude (Kliore, Moroz, and Keating 1985). The first OIR vertical profiles measured at the northern cold collar showed it extending from 65 to up to $90 \mathrm{~km}$ altitude (Schubert 1980), but VIRTIS measurements at the southern counterpart suggest it is concentrated at the 60-65 km level (Garate-Lopez et al. 2015; Grassi 2014) (Figure 26).

The cold collar has been defined in VIRA as an intermittent feature responsible for temperature inversions seen in individual soundings at latitudes from $50^{\circ} \mathrm{N}$ to $80^{\circ} \mathrm{N}$. It was not seen in some southern hemisphere profiles from Pioneer Venus (Kliore and Patel, 1982), nor was it detected in 
1802 the three northern hemisphere occultation profiles retrieved from Magellan (Jenkins et al., 1994). 1803 Its intermittent behavior has been confirmed by VIRTIS measurements, with the cold collar 1804 sometimes being absent in temperature maps (Garate-Lopez et al., 2015).

\subsection{Vortex dynamics}

The presence of solar tides in the cold collar latitudes has been analysed more recently in Peralta et al. (2012). OIR detected a diurnal tide at the northern cold collar between $70^{\circ} \mathrm{N}$ and $80^{\circ} \mathrm{N}$ (Schofield and Diner 1983; Schofield and Taylor 1983). A diurnal tide was also observed in northern hemisphere Venera-15 data for temperature and aerosol concentrations (Zasova et al. 2002). Radio-occultation data from VEx/VeRa, however, did not detect an $n=1$ structure, but a wavenumber $n=2$ (semidiurnal) mode at $\sim 62 \mathrm{~km}$, with a wave amplitude of $15 \mathrm{~K}$ and with minima at the subsolar and antisolar points (Tellmann et al., 2009). Peralta et al. (2012) suggest caution when interpreting this specific result from VeRa since, due to dataset coverage limitations, the sample used for the sine fit had only 12 bins and the data had a high dispersion.

Based on VEX/VIRTIS cloud tracking global wind measurements at $5.0 \mu \mathrm{m}(\mathrm{z} \sim 65 \mathrm{~km})$, Peralta et al. (2012) detected a dominant diurnal tide harmonic with an amplitude of about $4.7 \mathrm{~m} \mathrm{~s}^{-1}$ affecting the meridional component of the wind and forcing a solar-to-antisolar circulation across the pole. A quarter-diurnal mode has also been inferred, but it was only apparent in the zonal wind at $72^{\circ} \mathrm{S}$ latitude with $2.2 \mathrm{~m} \mathrm{~s}^{-1}$ amplitude.

The southern polar vortex was first detected as a double spiral dipole feature in VEx/VIRTIS 5 $\mu \mathrm{m}$ radiance (Piccioni et al., 2007). The temperature difference between the warmest part of the core and the cold collar has been measured to vary between 40 and $50 \mathrm{~K}$ (Piccioni et al., 2007, Garate-Lopez et al., 2015). The vortex core is a time-varying structure, however. Its position, morphology and rotation rate have been shown to change significantly in the short 24h-period of the VEx orbit (Figure 27). 
A
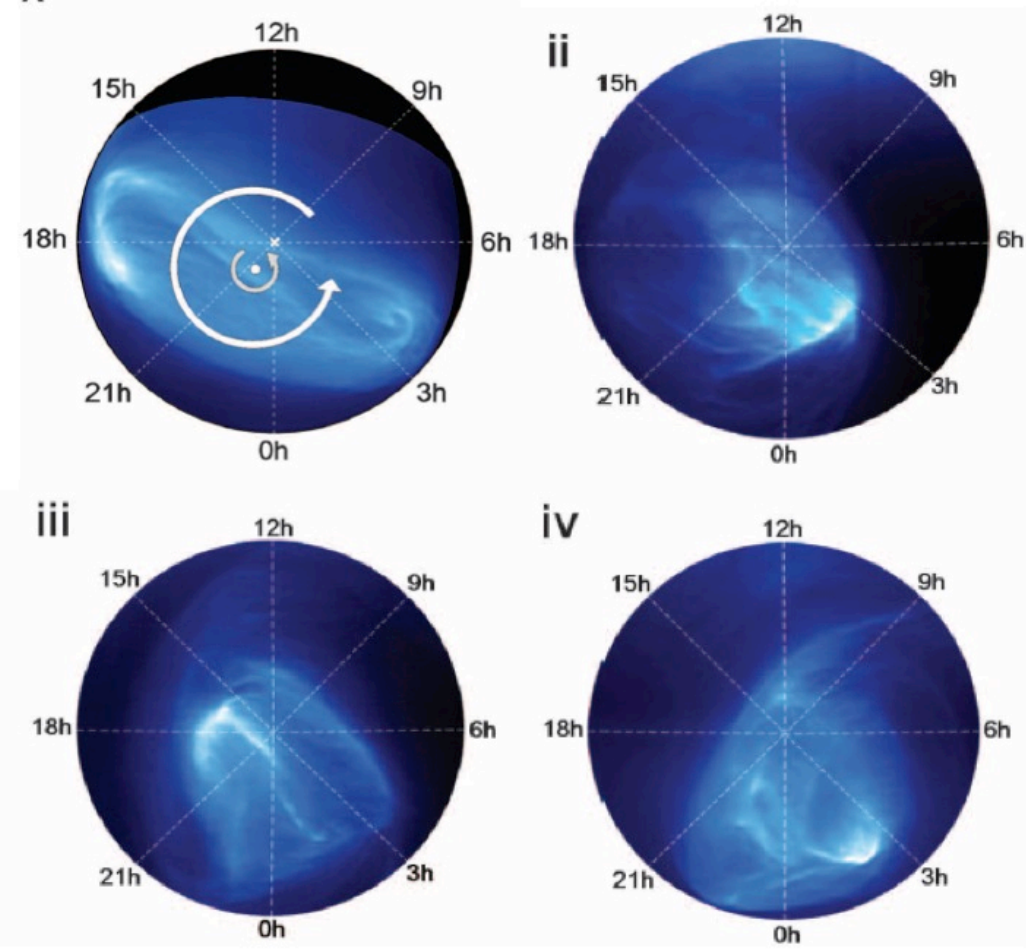

1832

1833

1834

1835

1836

1837

1838

1839

1840

1841

1842

1843

1844

1845

1846

1847

1848

1849

1850

1851

1852

1853

1854

1855

1856

Figure 27. VEx/VIRTIS radiance maps for the upper cloud (65 km) in latitude-local solar time coordinates, showing complex morphologies of the southern polar vortex during orbits 038 (i), 474 (ii), 475 (iii) and 476 (iv). In the top left panel the arrows indicate the motion of the feature around a centroid of rotation (white dot) displaced from the planetary axis (white cross). The outer circle is the $75^{\circ} \mathrm{S}$ parallel.

In the southern hemisphere, the $5 \mu \mathrm{m}$ bright vortex core is generally confined between $90^{\circ} \mathrm{S}$ and $80^{\circ} \mathrm{S}$. The basic core morphology is that of a single or double spiral (dipole), or (more rarely) a triple spiral, with filaments extending outwards from its center or connecting two or more brightness centers. The general form is that of a figure of bright filaments swirling around the pole. The figure is contained in a region of $\sim 2500 \mathrm{~km}$ diameter around the pole, with the centroid of vortex core rotation being confined within $80^{\circ} \mathrm{S}$ (Piccioni et al., 2007; Luz et al., 2011). Warm filaments rarely extend beyond the $75^{\circ} \mathrm{S}$ latitude circle.

Ground-based observations at 8.66 and $11.34 \mu \mathrm{m}$ (Sato et al., 2014) showed the polar features (hot polar spots and surrounding cold collars) in the northern and southern hemispheres apparently synchronized in their westward rotation. If confirmed, this synchronous motions between the two polar vortices would imply an important constrain for the Venus GCMs.

Cloud tracking wind measurements have shown the centroid of rotation of the vortex core to be displaced from the pole on average by $3^{\circ}$ (Luz et al., 2011). Furthermore, sequential measurements over four orbit sequences extending over periods between 4 and 10 days indicate the centroid of rotation to evolve around the pole in a precessional motion at a rate between 2.3 
and 4.9 hours/24h period in the local solar time frame, with an average motion of $\sim 52 \%$ day in the sense of the atmospheric rotation. In two of the sequences there was indication that the precession rate was not constant, with a secondary motion being present (Figure 27).

Different retrograde rotation rates were also measured for the inner vortex core and outer annulus of the vortex, with periods of 2.3+/-1.9 $\mathrm{d}$ for the inner core (between $90 \mathrm{~S}$ and $85 \mathrm{~S}$ ), and 2.8+/-1.9 $\mathrm{d}$ for the outer annulus (between $80 \mathrm{~S}$ and 85S). The average rate was $2.6+/-1.9 \mathrm{~d}$ for the extended vortex region circulation, in accordance with the value 2.48+/-0.05 d obtained shortly after orbit insertion (Piccioni et al., 2007) and not far from Pioneer Venus measurements of the northern dipole rotation period of 3 days (Schofield and Diner, 1983). Further measurements by Garate-Lopez et al. (2013) at $\sim 42 \mathrm{~km}$ and $63 \mathrm{~km}$ altitude obtained a mean rotation period of the vortex of $2.2+/-0.2 \mathrm{~d}$, and also confirmed the confinement of the centroid of rotation within $80^{\circ} \mathrm{S}$ and its wandering motion around the pole. However, their independent analysis of one of the orbit sequences did not confirm the precessional motion. Instead, they reported a chaotic meandering of the vortex center of rotation around the pole, with different positions of the centroid at the two altitude levels sounded. These common measurements should be interpreted with caution since different methods were used for determining centroids of vortex rotation. While in one case it was based on automated measurements of the wind field, which were subsequently interpolated (Garate-Lopez et al., 2013), in the other the centroid positions were determined visually by determining the point of least motion in animated time sequences of images (Luz et al., 2011) (Figure 27 and 29).

Modeling studies of polar vortex temperatures and dynamics fall into two major categories: GCM studies, in which the polar region is modeled as part of the global atmosphere (see below, section 8.2) and idealized vortex studies relying on barotropic models (Limaye et al., 2009; Elson 1982; Dobrovolskis and Diner, 1990; Michelangeli et al., 1987) (see Figure 28). Current GCMs qualitatively capture the main global features of the polar region, such as the warm feature above the pole and the thermal inversion, but with varying degrees of accuracy in the horizontal spatial extension of the features, temperature contrast and their height (section 8.2). However, the cold collar remains problematic and there is no satisfactory simulation that reproduces this feature. Idealized models of the vortex, on the other hand, are based on the barotropic vorticity equation and examine the non-linear evolution of the vorticity in the presence of a zonal flow compatible with observations (Hartmann 1983; Schubert 1999; Hoskins et al., 1985). These models successfully reproduce wavenumber-2 structures when initialized with barotropically unstable wind profiles, either inferred from the temperature field (Elson 1982; Michelangeli et al., 1987) or from direct wind measurements (Dobrovolskis and Diner, 1990; Limaye et al., 2009).

Explicitly, Travis (1978) and Elson (1978) found that high latitude jet-like features close to the edge of the polar vortex could be barotropically unstable in association with critical layers on either the poleward or equatorward sides of the high latitude jet, with maximum growth rates at zonal wavenumbers $\sim 1-2$. The most unstable mode peaked in amplitude close to the pole at a latitude $\sim 70^{\circ}$ and with a rotation period of $\sim 3.2$ days. These results were later confirmed in a divergent, linearized barotropic flow model by Dobrovolkis \& Diner (1990), who also suggested that the full nonlinear development of the instability may be essential to account for the detailed form of the observed polar dipole. 


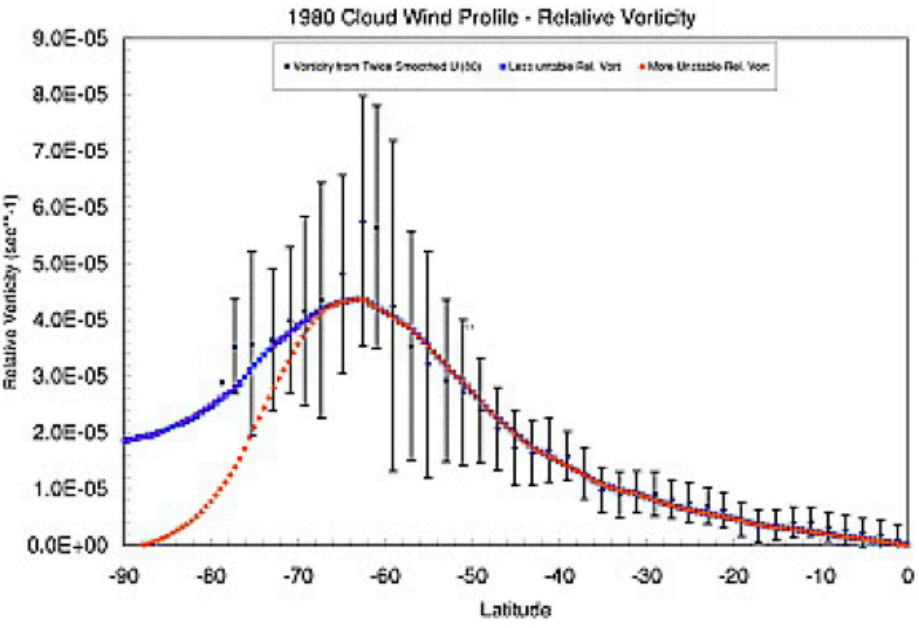

(a)

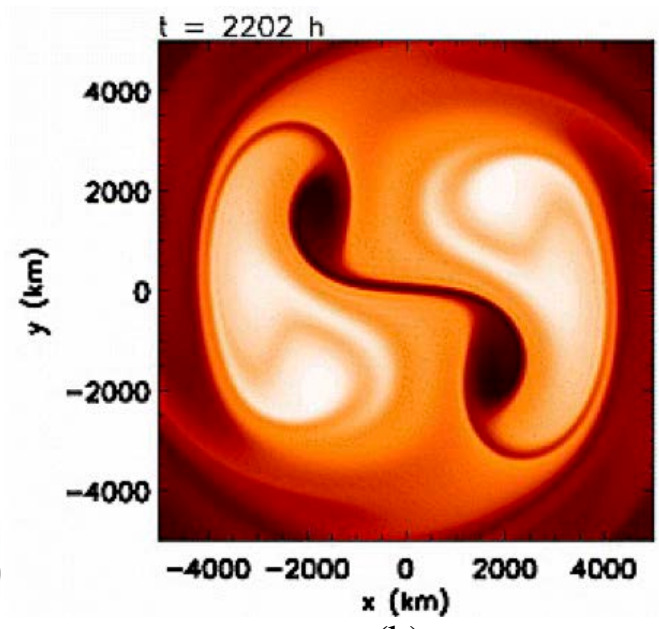

(b)

Figure 28 (a) Meridional profiles of relative vorticity close to the north polar vortex of Venus, obtained from Pioneer Venus UV images (points with error bars) and idealized in smooth profiles by Limaye et al (2009); (b) finite amplitude zonal wavenumber $n=2$ equilibrated barotropic instability, obtained in the numerical model of Limaye et al. (2009) after 2000 days initialized from the red profile shown in (a).

Such nonlinear development of the instability was investigated by Limaye et al. (2009), who used a fully nonlinear, non-divergent barotropic numerical model to study the evolution of barotropically unstable, zonally-symmetric initial states, derived from Pioneer Venus observations. Figure 28(a) shows examples of the relative vorticity profiles used as initial conditions, both of which exhibit a maximum at the edge of the polar vortex at a latitude of around $65^{\circ} \mathrm{N}$. These (and similar) profiles were found to be linearly unstable with zonal wavenumber $n=2$ disturbances having the fastest growth rates, with e-folding timescales $\geq 7.7$ days. The precise value depended upon the assumed vorticity values close to the pole, which are not well constrained by observations (e.g. Limaye et al. 2009, Garate-Lopez et al. 2013). The subsequent evolution of the flow (without explicit forcing to maintain the zonal flow) led to the development of a pair of vortices, connected by an "S"-shaped filament of vorticity, that proceeded to rotate around the pole while rolling up into ever tighter spirals, as illustrated in Figure 28(b). This feature forms from the vortex breakup in the nondivergent barotropic simulations, with its growth time depending on the degree of barotropic instability of the mean zonal wind profile (obtained from Pioneer Venus OCPP dayside observations in 1980 and 1982, with faster breakup occurring for the more unstable profile). The results bear a strong resemblance to some images of the polar dipole on Venus in the infrared (e.g. Piccioni et al. 2007), although the later development of the instability appeared less realistic as the "S"-shaped feature became less prominent and the (unforced) flow began to decay.

These results remain indicative, however, since they strongly depend on the instability of the mean wind profile caused by the presence of zonal jets close to $60^{\circ} \mathrm{S}$ (the modeled feature is about twice the size of the Venus dipole and rotates in about 4 days, while the actual rotation 
period is 3 days). The polar vortex is unlikely to be related to barotropic instability caused by jets at $60^{\circ} \mathrm{S}$, since the mean VEx/VIRTIS wind profile shows no evidence for them (Sánchez-Lavega et al. 2008; Hueso et al., 2012, 2015), although there are indications for mild occasional jets in instantaneous ground-based measurements made in 2007 (Machado et al. 2012). It is more likely that the polar vortex is created locally from barotropic or baroclinic instability within the inner boundary of the cold collar. Relative vorticity and divergence derived from direct cloud tracking wind measurements showed strong spatial variability (Garate-Lopez et al. 2013), which may locally induce barotropically unstable flow. Vertical winds in local sources or sinks at the cloud tops are on the order of $0.1 \mathrm{~m} \mathrm{~s}^{-1}$. The appearance of the vortex is similar when seen in the upper and lower clouds, at 5.0 and $1.74 \mu \mathrm{m}$ (Figure 29). In some orbits, the vertical shear of the zonal wind between these two altitudes reached magnitudes as high as $|d u / d z| \sim 0.8+/-0.2 \mathrm{~m} \mathrm{~s}^{-1} \mathrm{~km}^{-1}$, but its mean value is close to zero. With few exceptions, the thermal emission morphology showed no spatial correlation with the vorticity distribution, an indication that a direct connection between dynamics and thermal emission features is unlikely.

The dynamical interaction between the warm vortex core and the surrounding cold collar has only just begun to be investigated (Garate-Lopez et al. 2015). The cold collar is the most statically stable structure at night-time polar latitudes, while the warm vortex core and its hot filaments display successively lower stability values. The presence of the cold collar between 55 and $65 \mathrm{~km}$ altitude appears to confine the warm vortex to latitudes poleward of $75^{\circ} \mathrm{S}$, while at higher altitudes the vortex appears more extended (see Figure 26).
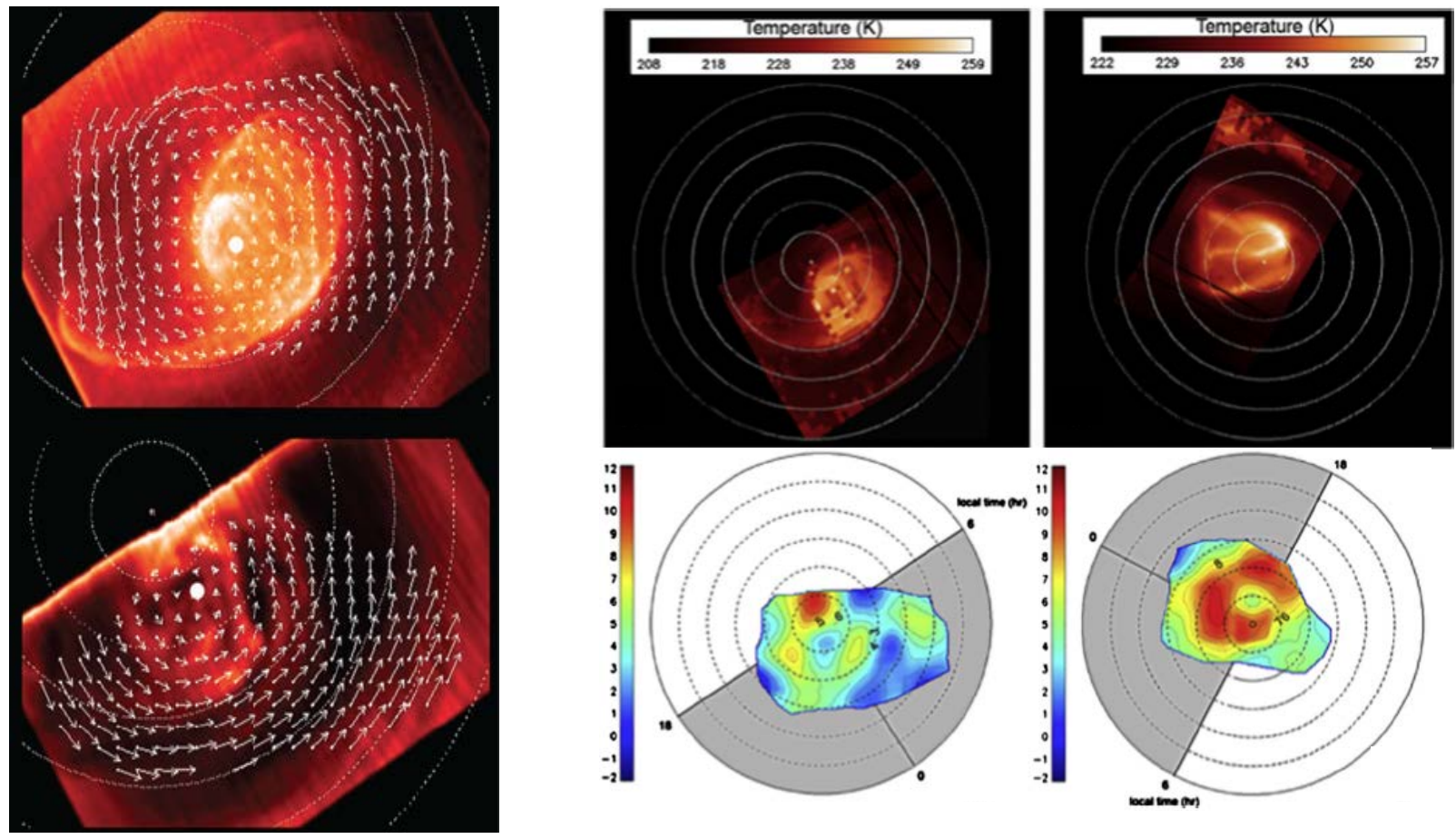

Figure 29. Left: Polar projections of vortex morphology as observed at infrared wavelengths for the upper cloud at $5.0 \mu \mathrm{m}$ (top) and for the lower cloud at $1.74 \mu \mathrm{m}$ (bottom), on orbit 310. White 
arrows represent cloud tracked zonal wind velocities. The largest vectors in each panel are $50 \mathrm{~m}$ $\mathrm{s}^{-1}$ and $46 \mathrm{~m} \mathrm{~s}^{-1}$ (Garate-Lopez et al. 2013). Middle and right panels: Temperature maps at the 360 mbar pressure level (top) for orbits 310 and 475, and maps of relative vorticity from cloud tracked winds for the same orbits (bottom) (Garate-Lopez et al. 2015).

The effect of the thermal tide on the polar vortex has been investigated by Yamamoto and Takahasi (2015). They showed, using a GCM, that the polar diurnal tide enhances the cold collar and the vortex core at cloud-top level, displacing the hot core from the pole and reinforcing the polar vortex in combination with the transient baroclinic wave. A different idea but also involving the thermal tide has been invoked by Ando et al. (2016). They also used a GCM to reproduce the characteristics of the polar vortex. According to their results the thermal tide enhances the residual mean meridional circulation that leads to the cold collar and the warm polar region.

\section{Global atmospheric models}

In this section, the theoretical framework developed to explain the circulation in Venus's atmosphere is reviewed, as well as the numerical models that have been built to decipher the super-rotation mechanism, and the respective roles of the different waves in the processes driving the observed features.

\subsection{The GRW mechanism and Hadley cells}

One of the earliest and most effective explanations for the strong atmospheric super-rotation of the Venus atmosphere has come to be known as the "Gierasch-Rossow-Williams (or GRW) mechanism”, following Gierasch (1975) and Rossow \& Williams (1979). In reality, this is not really a single "mechanism" but rather a class of conceptual dynamical scenarios that share some important features in common.

\subsubsection{A “Classical GRW” scenario}

The basic concept, as set out by Gierasch (1975), is partly founded on the effect of a thermallydirect, axisymmetric overturning circulation (in the form of hemispheric Hadley cells) to transport materially conserved variables upward and polewards. In its purest form, such a circulation will satisfy Hide’s theorem (see e.g. Read 2013)and lead to the spin-up of prograde zonal flow at upper levels in mid-latitudes as equatorial air moves polewards, approximately conserving its specific angular momentum while depleting the tropics of angular momentum. Gierasch (1975) then closed the problem by invoking a set of unspecified eddy processes, via a simple diffusive parameterization, to bring the angular momentum budget into balance with prograde equatorial zonal flow. However, it was necessary to make certain assumptions about the way eddies mix momentum, vorticity and heat in order that they should not remove thermal gradients or mix angular momentum in the vertical too effectively.

Gierasch (1975) placed an emphasis on the effects of eddies acting primarily as agents of the horizontal mixing of vorticity, which also enables eddy diffusion to transport angular momentum equatorwards and up-gradient, in a similar manner to horizontal molecular viscosity (Plumb, 
1977; Read, 1986). This emphasis on the role of eddies as horizontal vorticity mixers was taken up subsequently by Rossow and Williams (1979), who investigated the possible role of largescale, barotropically unstable eddies under Venus-like conditions. They also demonstrated numerical solutions of super-rotating flows in a shallow-water model. This combination of poleward angular momentum transport by axisymmetric Hadley circulations balanced by equatorward transport by eddies represents what might be termed the "classical GRW" mechanism, although there may be many problem-dependent variations on this theme in practice, depending upon the detailed eddy processes that might occur.

Angular momentum exchanges with the underlying surface are also crucial to the final steady state achieving a state of global super-rotation. This is because the atmosphere also needs to come into equilibrium with the underlying surface via a combination of torques due to frictional and turbulent exchanges in the boundary layer or topographic form-drag. The overall steady state can be understood with reference to the schematic diagram in Figure 30.

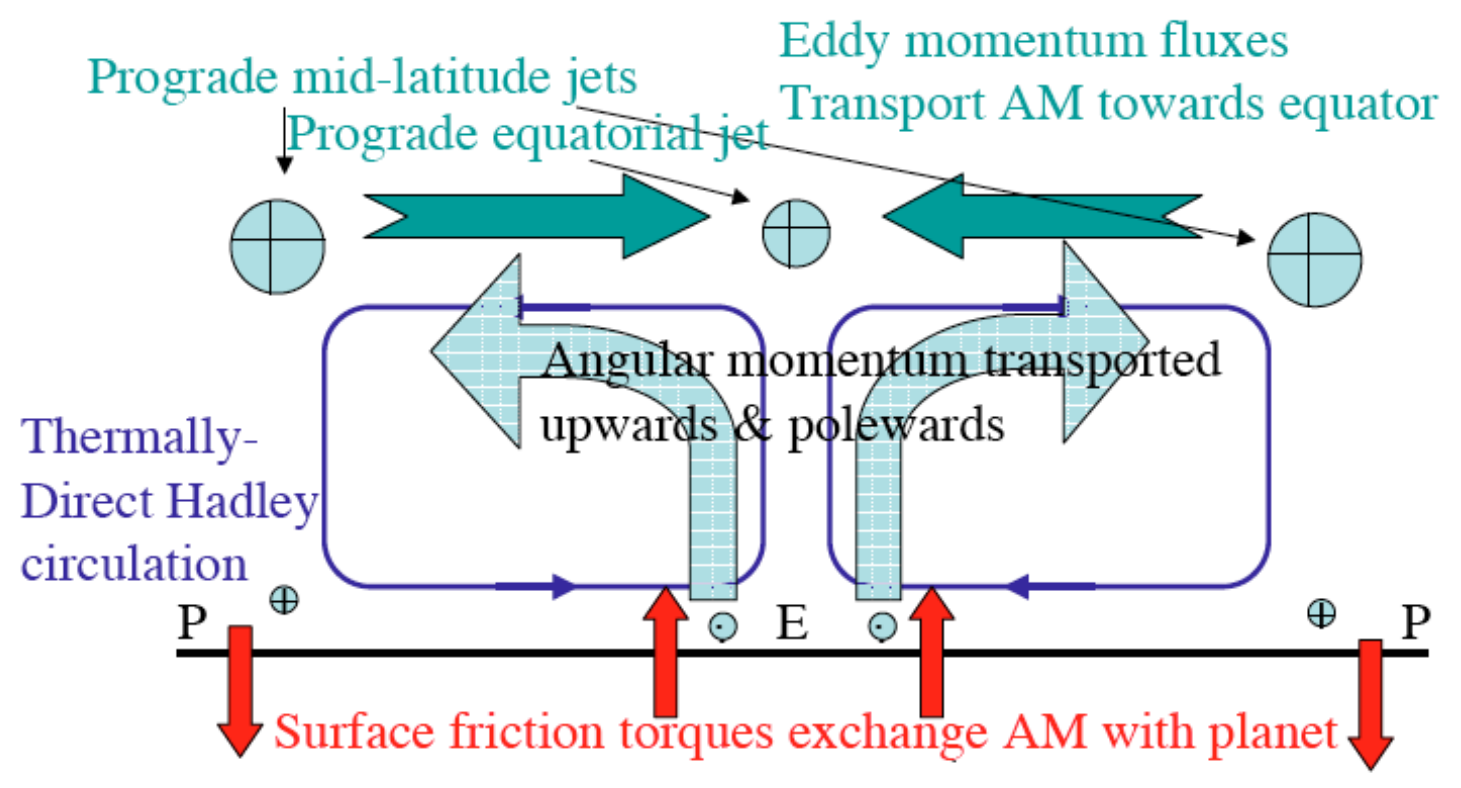

Figure 30 Schematic diagram illustrating the main features of the "classical" Gierasch-RossowWilliams scenario for atmospheric super-rotation. $P$ and $E$ denote the location of the poles and equator respectively. Circles with crosses indicate prograde zonal winds (in the same sense as the planet's rotation) and circles with dots indicate retrograde zonal flow.

This clearly suggests the formation of prograde mid-latitude zonal jets at upper levels, close to the poleward boundary of the Hadley cells in each hemisphere, where angular momentum nearconservation from the equator has spun up significant zonal flow. These are brought to an equilibrium at upper levels through the equatorward eddy fluxes which, at least in this simple conceptual model, deplete the mid-latitudes of angular momentum and are convergent on the equator to accelerate prograde flow. Near the surface, however, the prograde motion at midlatitudes tends to be decelerated by surface torques. Given the surface generally exerts a negative 
drag on relative flow, this implies retrograde flow close to the surface at the equator, so that the

\subsubsection{A “non-classical” GRW scenario}

An alternative, though somewhat related, scenario is illustrated in Figure 31. In this case, a thermally-direct Hadley circulation (i.e. a circulation transport heat directly from hot to cold) acts as before to extract angular momentum from the solid planet in the tropics during spin-up, and to transport specific angular momentum $m$ upwards and polewards to form mid-latitude jets. Unlike in the "classical" GRW scenario, however, in this alternative approach the eddies are assumed transporting $m$ pre-dominantly up-gradient in the vertical direction instead of in the horizontal. Such a configuration is equally valid in the context of Hide's theorem, supporting a local maximum in $m$ over the equator, provided any horizontal momentum fluxes are predominantly poleward instead of equatorward.

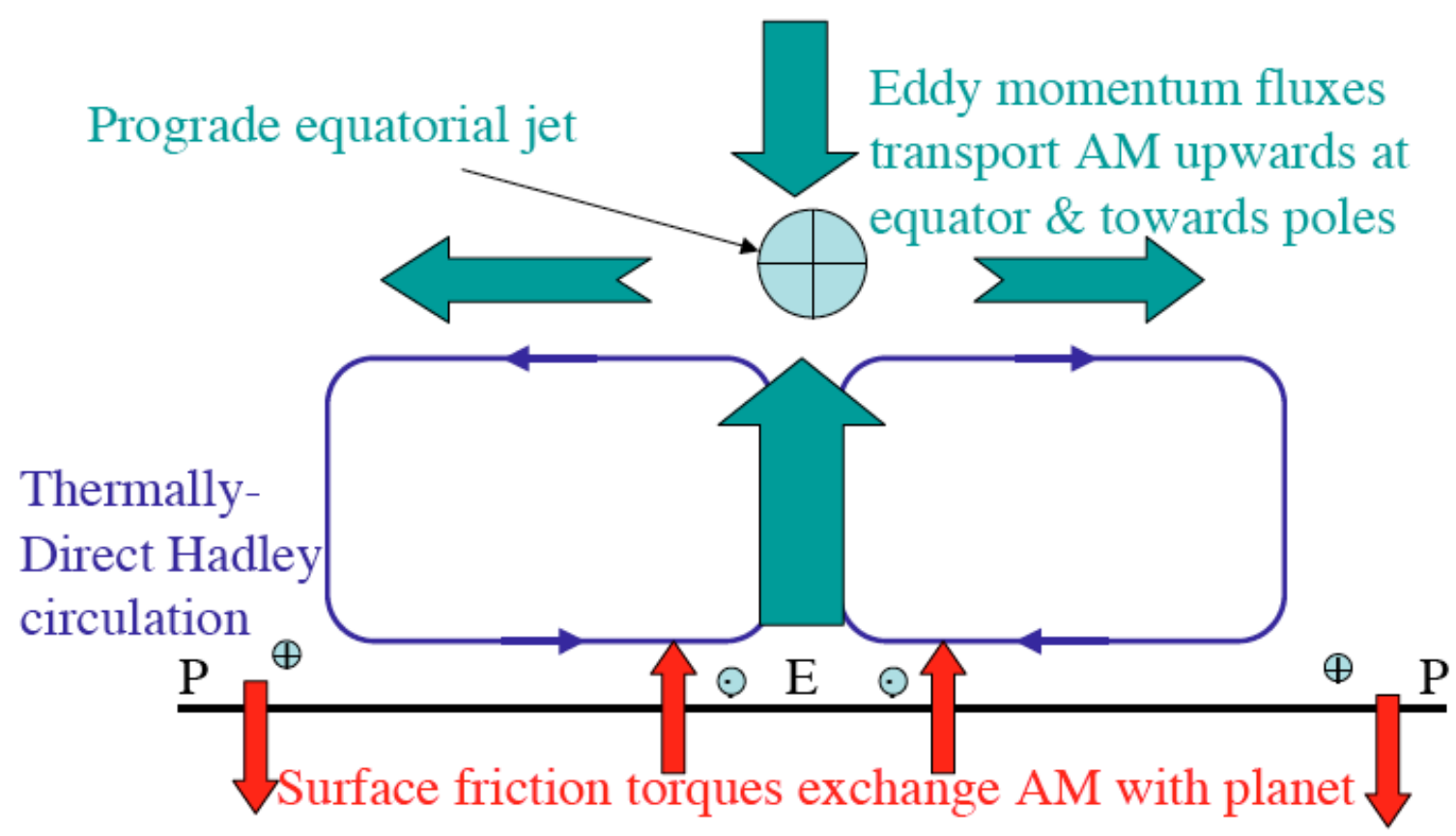

2057

2058

2059

2060

2061

2062

2063

2064

2065

2066

2067

2068

2069
Figure 31 Schematic diagram illustrating the main features of the alternative to the "classical" Gierasch-Rossow-Williams mechanism for atmospheric super-rotation, in which up-gradient fluxes of angular momentum occur predominantly in the vertical (see text). Symbols are the same as in Figure 30.

Eddies acting in this way are likely to have a quite different origin from the horizontal vorticity mixing, predominantly barotropic, eddies invoked in the "classical GRW" mechanism, but there are a number of plausible candidates. Thermal tides, for example, were shown to have components that transport angular momentum both upwards and downwards into the upper cloud layers on Venus in the simplified models of Leovy \& Baker (1987) and Newman \& Leovy (1992), mainly via the semi-diurnal tide, while the diurnal tide would produce poleward eddy 
fluxes of angular momentum, as discussed in Section 5 above. Other kinds of verticallypropagating waves, ranging from equatorial Kelvin-like modes and other inertia-gravity waves on a planetary scale (e.g. Peralta et al. 2014a,b,2015) to small-scale internal gravity waves, can also result in effective vertical transport of angular momentum, accelerating or decelerating zonal flow at levels where they are generated or dissipated (DelGenio and Rossow 1990; Imamura 2006; Kouyama et al. 2015; Yamamoto and Tanaka 1997). In this regard, these waves may act in a somewhat similar way to how upward-propagating waves from the Earth's troposphere drive the Quasi-Biennial Oscillation in the equatorial stratosphere (cf Baldwin et al. 2001).

Precisely which version of the GRW scenario is applicable as a conceptual model of Venus' global atmospheric circulation is still not conclusively established from direct observations. As discussed in section 2.3, the direction of the mean meridional circulation is largely poleward at the cloud tops, suggestive of the upper branch of hemispheric, thermally-direct Hadley circulations, but evidence is less conclusive at other heights. The detailed vertical structure of any putative Hadley circulation is therefore not well constrained by observations, though it is generally thought likely that at least one closed Hadley cell is present in each hemisphere. But whether a single circulation cell connects the cloud top levels with the surface, as in the original scenario proposed by Gierasch (1975), or a more complex vertical structure is present, is unclear (see Sections 3, 5.1 and 7.1 above).

Evidence for the origin, strength and direction of eddy transports necessary to close the GRW angular momentum transport cycle is equally ambiguous. As discussed in section 4, incomplete coverage of the day and nightsides in wind measurements can lead to biases that may distort the interpretation of eddy correlations. Direct observations suggest fluxes that are predominantly poleward (Limaye et al. 1982; Rossow et al. 1990), though these are mostly from the dayside. As argued by Limaye (2007), however, taking into account incomplete nighttime coverage suggests that the horizontal eddy transports near the cloud tops may fluctuate in sign between poleward or equatorward angular momentum transport at different seasons and altitudes. Direct evidence of vertical transports are even more difficult to come by, though vertically-propagating tides and gravity waves have been observed directly (see sections 4.2 and 5).

Ultimately, however, the ability of fully self-consistent dynamical models to reproduce observed properties is likely to be the most powerful means of exploring these kinds of question concerning the overall scenario for Venus' global circulation for the foreseeable future. Such models have been growing substantially in sophistication in recent years, and are reviewed in the following sections.

\subsection{General Circulation Models}

To study atmospheric dynamics, General Circulation Models are very efficient tools. Their recent improvements in the case of Venus atmosphere has now brought significant results. However, there are issues associated with these tools that need to be taken into account. A recent review of the historical efforts achieved in Venus atmospheric modeling has been published in the frame of a working group sponsored by the International Space Science Institute (ISSI, in Bern, Switzerland) during the period 2008-2012 (Lewis et al., 2013). This review mentioned 
only those modeling studies applied to the atmosphere from the surface to roughly $100 \mathrm{~km}$ altitude, where the circulation is dominated by the super-rotation. Above this level, non-LTE radiative processes become dominant and control the thermal structure, while the zonal circulation makes a transition from super-rotation to a thermospheric sub-solar to anti-solar circulation.

\subsubsection{First developments}

For the last 50 years, General Circulation Models (GCMs) have been developed to model the Earth's atmosphere. These tools solve the fluid dynamical equations for a spherical atmosphere, with variable temporal and spatial resolutions. The development of these models has followed the increasing possibilities provided by the reduction of the computation time, now reaching the state of very evolved simulation tools that couple the ocean, atmosphere and biosphere.

The exploration of the Venus's atmosphere by Russian and American spacecrafts since the 1970s has provided a strong motivation to adapt Earth GCMs to Venus conditions. The first model developed for the atmospheric circulation of Venus was published by Kalnay de Rivas (1975). This was a 2-dimensional (latitude-altitude) model, extended in the longitudinal dimension by adding longitudinal wave terms. The resolution was limited to 18 latitudes, and the simulation was made only for one Venus rotation (roughly 240 Earth days). The circulation obtained with this model showed two equator-to-pole Hadley-type cells. Young and Pollack (1977) published a GCM that apparently nicely reproduced available observations at the time. In the vertical, this GCM had 16 to 32 levels, equally distributed from the surface to $64 \mathrm{~km}$ altitude, with a finitedifference scheme. In the horizontal, the equations were solved with spherical harmonics (i.e. a pseudo-spectral model). Rossow et al. (1980) argued, however, that this GCM had significant problems arising from poor angular momentum conservation, as well as from the truncation used and its assumed symmetry, which eliminated most of the wave modes.

During the early years of the Pioneer Venus mission, two GCM studies were published. Rossow (1983) adapted the GFDL (Geophysical Fluid Dynamics Laboratory) terrestrial GCM to Venus conditions. This spectral model used a horizontal truncation of 15 wavenumbers on 9 vertical levels. Simulations run for more than 5000 Earth days produced zonal winds 3 to 5 times slower than observed. Mayr and Harris (1983) used also a spectral GCM to study the super-rotation phenomenon, with emphasis on Venus. Varying many of the model parameters, they could obtain in one case zonal winds up to $\sim 100 \mathrm{~m} / \mathrm{s}$.

\subsubsection{Venus General Circulation Models: simplified thermal forcings}

One of the most demanding requirements to develop a realistic simulation of Venus's atmospheric circulation is to perform the radiative transfer calculations needed in the GCM. This is challenging in Venus's conditions because of the very opaque cloud layer and dense $\mathrm{CO}_{2}$ atmosphere, with very high temperatures and pressures below the clouds. That difficulty may explain why, when a renewed effort started around 2000 to develop Venus GCMs, these models used simplified temperature forcing with Newtonian cooling, where cooling rates are computed through the relaxation of the temperature towards a prescribed profile. 
In Japan, a sustained effort is on-going in several teams, exploring many aspects of Venus's atmospheric system. Based on a spectral dynamical core developed at the Center for Climate System Research / National Institute for Environmental Studies (CCSR/NIES), Yamamoto and Takahashi developed a Venus GCM extending from the surface to roughly 90-100 km (on 50 levels for the first model, then 52 and even 72 levels for the latest model), with a horizontal resolution starting at 32x16 (T10 spectral truncation, Yamamoto and Takahashi, 2003a - i.e. a triangular truncation in wavenumber space at total wavenumber 10) then evolving to $64 x 32$ (T21, Yamamoto and Takahashi, 2003b, 2004, 2006, 2009, 2012). The thermal structure was forced with a meridional distribution that was fixed for the solar heating rate, including latitudinal variations, and Newtonian cooling with relaxation towards a prescribed temperature profile. A variation of the same GCM was also used by Kido and Wakata (2008).

A different kind of forcing was used by Takagi and Matsuda (2007) for their GCM, based on a low spectral resolution (T10) dynamical core (with 50 vertical levels from the surface to $90 \mathrm{~km}$ ). Instead of a zonally averaged solar forcing, however, they used only the tidal component of the solar heating rates, to focus on the effect of thermal tides on the global circulation.

At the University of Oxford around the same period, Lee et al. (2005, 2007) also developed a simplified Venus GCM, based on the UK Meteorological Office Unified Model, a finitedifference grid-point model with 32 vertical levels $(0-90 \mathrm{~km})$ and a horizontal resolution of $5^{\circ} \times 5^{\circ}$ in latitude and longitude. In this model, the radiative forcing was entirely controlled by the Newtonian cooling, with the temperature relaxation profile depending on latitude and height. In Great Britain again, at the Open University, Lewis et al. (2006) used a pseudo-spectral dynamical core for their Venus GCM, with T21 to T42 horizontal resolution and 32 to 200 vertical levels $(0-100 \mathrm{~km})$. In the meantime, in the United States, Hollingsworth et al. (2007) adapted another terrestrial GCM, from the NASA Goddard Space Flight Center ARIES/GEOS dynamical core. The set-up was similar to the Yamamoto and Takahashi (2003b, 2004) experiments. The same group later started to work with another GCM, the NCAR Community Atmospheric Model (CAM), which had a finite-volume dynamical core and required a much higher horizontal resolution to be used $\left(\sim 1^{\circ} \times 1^{\circ}\right)$ because of dissipation issues (Parish et al., 2011).

The first GCM considering the topography of the Venus's surface was the GCM EPIC Herrnstein and Dowling (2007), which used isentropic, terrain-following hybrid vertical coordinates. The thermal forcing in this GCM was similar to Lee et al. (2005). Finally, Lee and Richardson (2010) developed a specific approach for their Venus atmospheric experiments: they used three different versions of the dynamical core of the Geophysical Fluid Dynamics Laboratory (GFDL) terrestrial GCM (finite-differences, spectral, and finite-volume cores) with the same simplified physical forcings, similar to those used by Lee et al. (2005, 2010).

Most of the above simulations were able to produce a realistic super-rotation, with the zonal wind being always maximum near the cloud tops between equator and roughly $60^{\circ}$ latitude, and peaking in a jet generally located near $50-60^{\circ}$ latitude. The amplitude of these peaks depended upon the model, e.g. $\sim 40 \mathrm{~m} / \mathrm{s}$ for Lee et al. (2005), up to $120 \mathrm{~m} / \mathrm{s}$ for Yamamoto and Takahashi (2003b). The meridional circulation obtained exhibited two equator-to-pole Hadley-type cells. 
However, to obtain a fully developed super-rotation, Yamamoto and Takahashi (2003a,b) had to tune their heating rate parameters. They note that the maximum in the heating rates needed to be lower by roughly $10 \mathrm{~km}$ than the cloud-top. Also, they used a forcing that included both an equator-to-pole temperature contrast and a deep-atmosphere heating rate that was much higher than expected from available observations. This problem was confirmed by various studies (Lee et al., 2005; Yamamoto and Takahashi, 2006, 2009; Hollingsworth et al., 2007), noting that the super-rotation was easily lost without specific tuning of the forcing parameters. The impact of changing the deep-atmosphere heating rates was studied in Hollingsworth et al. (2007) and Yamamoto and Takahashi (2009). Realistic values for heating rates in the deep atmosphere are of the order of $10^{-3} \mathrm{~K} /$ day (Tomasko et al., 1980), but to obtain super-rotation, Hollingsworth et al. (2007) had to use a value of $0.5 \mathrm{~K} /$ day (without including thermal tides), while Yamamoto and Takahashi (2009), who included thermal tides, explored the sensitivity to various values and obtained a realistic super-rotation for heating rates of the order of $0.1 \mathrm{~K} /$ day. Yamamoto and Takahashi (2006) studied the sensitivity to the latitudinal temperature contrast set at the surface. When set to $10 \mathrm{~K}$, the super-rotation was realistic but when decreased to $5 \mathrm{~K}$, the super-rotation was significantly decreased. Observational constraints indicate a latitudinal temperature contrast lower than $5 \mathrm{~K}$ in the deep atmosphere. Lee et al. (2005, 2007) also had to force a temperature contrast superior to $6 \mathrm{~K}$ in the deep atmosphere to obtain a strong super-rotation.

Wave activity and its role in the resulting super-rotation were also studied in these works. Equatorial Kelvin waves were analyzed near the cloud-tops, with periods varying with the amplitude of the super-rotation, but similar to the observed 4-day period. Mixed RossbyGravity waves were also found (e.g. Lee et al., 2005, 2007). By including the thermal tides, Yamamoto and Takahashi (2004) found them to play a significant role in the horizontal transport of angular momentum towards the equator. In their analysis, when including only the timevarying part of the heating rate, Takagi and Matsuda (2007) showed that the thermal tides were transporting angular momentum vertically, thus participating in the production of super-rotation in the equatorial region. In their recent paper, Yamamoto and Takahashi (2012) used a different forcing for the middle atmosphere, in order to evaluate the role of planetary scale waves on the super-rotation. They focus on the atmosphere above $30 \mathrm{~km}$, and forced the circulation with planetary scale, equatorial Kelvin-type waves at $30 \mathrm{~km}$ altitude with periods 4-6 days and zonal wavenumber 1 . They found the amplitude of the maintained super-rotation was sensitive to the strength of these waves, which also affected the dynamics of the polar vortex.

The most recent GCM based on this type of forcing was designed and published by Sugimoto et al. (2014a, 2014b). It is based on the Atmospheric GCM for the Earth Simulator (AFES). The design of the simulations includes a Newtonian relaxation towards a latitudinally dependent temperature distribution (as in e.g. Lee et al., 2005). In Sugimoto et al. (2014b), latitudinally dependent solar heating is added to the Newtonian cooling (as in e.g. Yamamoto and Takahashi, 2003a,b), including a diurnal cycle. The initial state is taken as already in super-rotation, and the initial temperature distribution is set to be in balance with this initial zonal wind field. But the initial profile of static stability is forced towards a realistic profile. The spectral core is used at relatively high resolution (T42L60 in Sugimoto et al. 2004a, T63L120 in Sugimoto et al. 2004b), with a vertical domain extending up to $\sim 120 \mathrm{~km}$. The super-rotation is maintained in the simulations over 15 to 30 Venus days, with a zonal wind distribution that is closer to observations with this type of forcing (compared to a simple Newtonian relaxation), as illustrated 
2254

2255

2256

2257

2258

2259

2260

2261

2262

2263

2264

2265

2266

2267

2268

2269

2270

2271

2272

2273

2274

2275

2276

2277

2278

2279

2280

2281

2282

in Figure 32. The authors study mainly the role of baroclinic instabilities that develop in midlatitudes around $60 \mathrm{~km}$ altitude and generate wavenumber 1 perturbations at the cloud top, with a period around 5.8 Earth days, similar to Rossby-type waves. The circulation includes also equatorial barotropic waves below the bottom of the clouds, but no Kelvin-type wave was obtained at the cloud top.

(a) $\overline{U^{\prime} V^{\prime}}$

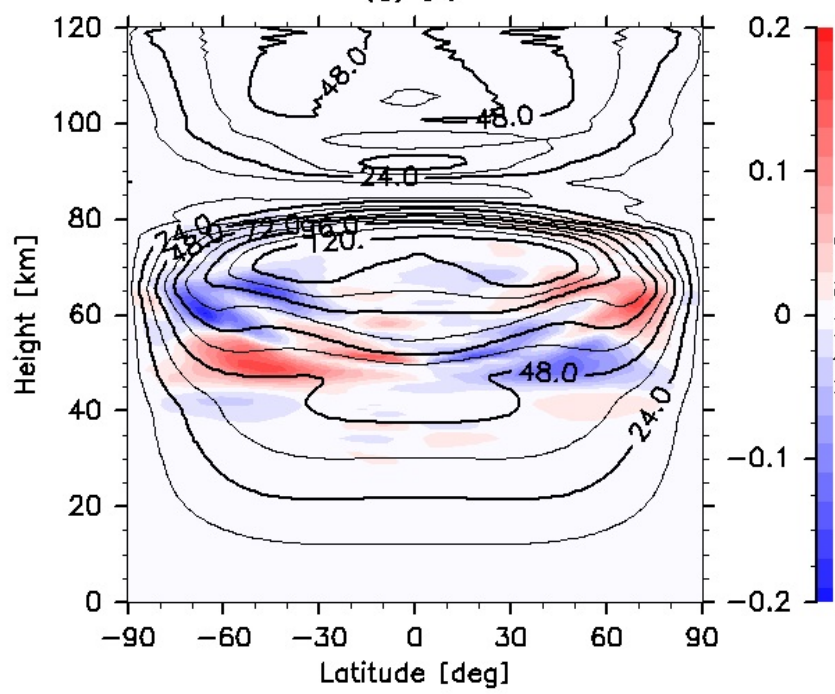

CONTOUR INTERVAL $=1.200 E+01$ (b) $\overline{V^{\prime} T^{\prime}}$

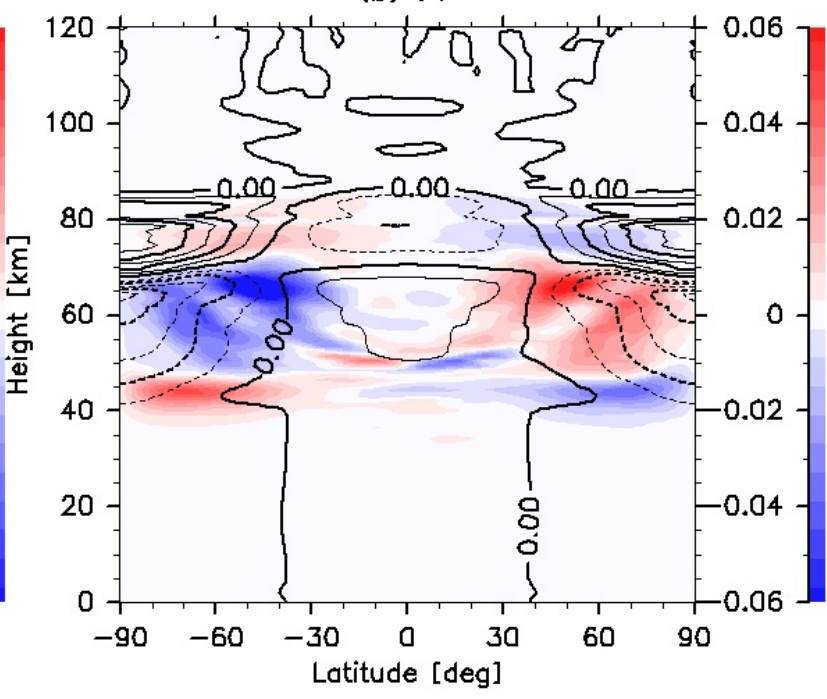

CONTOUR INTERVAL $=4.000 E+00$

Figure 32. Simulations obtained in the Venus AFES simulations of Sugimoto et al. (2014b). Distributions of (a) momentum flux $u^{\prime} v^{\prime}$ and (b) heat flux $v^{\prime} T^{\prime}$, averaged over 90 Earth days in latitude-height cross sections. The amplitudes are pressure-weighted. The zonal-mean zonal flow (a) and temperature deviations from the horizontally averaged temperature (b) are also shown in black contours. Reproduced from Sugimoto et al. (2014b).

\subsubsection{Radiative transfer developments}

Though simplified temperature forcing allowed modellers to explore Venus's atmospheric dynamics, the development of full radiative transfer models that could be implemented in GCMs had to be investigated to go forward to more realistic simulations. To model the atmospheric temperature structure of Venus correctly, the determining inputs are the opacity distribution and an a priori temperature profile, usually based on the Venus International Reference Atmosphere (VIRA, Kliore et al., 1985). The computation of opacities is a crucial part of the radiative transfer modeling. These are determined by the cloud structure and properties, the gas composition of the atmosphere, and the spectral properties of the different gas constituents.

A first effort to include a full radiative transfer computation in a GCM was reported with the PhD study of K. Ikeda (Ikeda et al., 2007; Ikeda, 2011). The formulation is based on discrete ordinate and adding methods, in a delta two-stream approximation. The spectrum from 0.2 to $1000 \mu \mathrm{m}\left(10-50000 \mathrm{~cm}^{-1}\right)$ covers both solar and thermal radiation and is divided into 28 bands. This radiative transfer scheme was implemented in a Venus version of the CCSR/NIES GCM with a T21 horizontal resolution and 52 levels $(0-90 \mathrm{~km})$, similar to the model used by 
Yamamoto and Takahashi (2006). The temperature profile obtained was close to the VIRA profile below $40 \mathrm{~km}$, but was colder above, mainly in the cloud layer. This GCM produced a fair super-rotation in the cloud layer, with a peak of $40-50 \mathrm{~m} \mathrm{~s}^{-1}$ at the cloud top, but very low wind speeds below $50 \mathrm{~km}$ (less than $5 \mathrm{~m} \mathrm{~s}^{-1}$ ).

Around the same period, a different type of approach was investigated for the GCM that was developed in France. Eymet et al (2009) used a Net Exchange Rate (NER) method to compute the radiative transfer in the infrared wavelength range. In this formalism, the monochromatic radiative budget of a given atmospheric layer is computed as the sum of the radiative net exchanges between this layer and all other atmospheric layers, including the boundaries, i.e. the surface and space. The wavelength domain used goes from 1.71 to $250 \mu \mathrm{m}\left(40-5700 \mathrm{~cm}^{-1}\right)$, divided into 68 narrow bands of variable width. The net exchange rate matrix is computed with a Monte-Carlo code, assuming the temperature profile from VIRA. The dependence of this matrix on the temperature profile is extracted assuming the NER between two layers is equal to the difference in the Planck functions for these two layers, multiplied by an optico-geometric exchange coefficient that does not depend on temperature. The matrix containing only these exchange coefficients can then be used within the radiative transfer module of a GCM to compute the temperature profile self-consistently.

This method was implemented in the Venus GCM developed at the Laboratoire de Meteorologie Dynamique (LMD) in Paris (Lebonnois et al., 2010, 2016). This GCM is based on the LMDZ finite-difference dynamical core developed at LMD for the Earth and used also for Mars, Titan and other applications. The Venus GCM simulations from Lebonnois et al. (2010) used a horizontal resolution of 48 longitudes by 32 latitudes, on 50 vertical levels (from the surface to roughly $95 \mathrm{~km}$ altitude), while in the latest simulations (Lebonnois et al., 2016), the horizontal resolution was increased to 96x96. In addition to the infrared radiative transfer from Eymet et al. (2009), solar heating is computed from look-up tables that depend upon solar zenith angle, provided by the Crisp (1986) model. The drawback of this infrared radiative transfer method is that taking into account the variability of the opacity sources is difficult. A fixed latitudinal dependence of the cloud structure may be implemented, but an interactive dependence of the exchange coefficients to the opacity distribution during the GCM simulation is difficult to consider. In Lebonnois et al. (2010, 2016), the cloud is then considered as fixed and with a vertical distribution that is uniform over the horizontal domain. In these simulations, the diurnal cycle was included, topography was taken into account, as well as the dependence on temperature of the specific heat capacity, $C_{p}(T)$, that is significant in the atmosphere of Venus and affects the definition of the potential temperature used within the dynamical core equations.

In Lebonnois et al. (2010), simulations were run both with and without topography, and with either the full radiative transfer or a simplified temperature forcing (similar to the one of Lee et al., 2005) to estimate the sensitivity of the circulation to the radiative scheme. The results with the simplified temperature forcing (with no diurnal cycle) were broadly consistent with earlier studies, described in the previous section, with peak zonal winds (less than $40 \mathrm{~m} \mathrm{~s}^{-1}$ ) at midlatitudes and near the bottom of the clouds, and two equator-to-pole Hadley-type cells covering the whole vertical domain of the GCM. The simulations with full radiative transfer, however, induced a quite different circulation. The zonal wind peaked at the cloud top in the equatorial region, with values around $60 \mathrm{~m} \mathrm{~s}^{-1}$, and the meridional circulation was divided into vertically 
stacked Hadley-type cells, one in the atmosphere below the clouds, one within the cloud region, and one above. The zonal wind below the clouds remained very small (below $5 \mathrm{~m} \mathrm{~s}^{-1}$ ) in these simulations. The budget of angular momentum was analysed and showed that the super-rotation builds up and was maintained through a mechanism similar to GRW, but the role of thermal tides was clearly emphasized: diurnal and semi-diurnal tides transported angular momentum vertically in the equatorial region, above the cloud top, and participated in the zonal wind maximum located at the equator. The role of thermal tides in the angular momentum budget was also noticed in Ikeda (2011).

In later work, the planetary boundary layer scheme was improved in the LMD Venus GCM, which affected the static stability profile above the surface and resulted in an increase of the zonal wind in the deep atmosphere, with positive values everywhere except close to the equatorial surface and values above $5 \mathrm{~m} \mathrm{~s}^{-1}$ in the 20-45 km region. These results showed a thermal structure in the upper cloud and above dominated by thermal tides, which was compared with VIRTIS-H/Venus Express temperature retrievals in Migliorini et al (2012). With this improved boundary layer scheme and by increasing the horizontal resolution, Lebonnois et al. (2016) have improved the agreement between observed and modeled zonal wind field (Figure 33). In this work, the wave analysis performed confirms the role of thermal tides in the angular momentum budget and the presence of baroclinic wave activity in the cloud layer, as in the 2349 simulations by Sugimoto et al. (2014a,b).

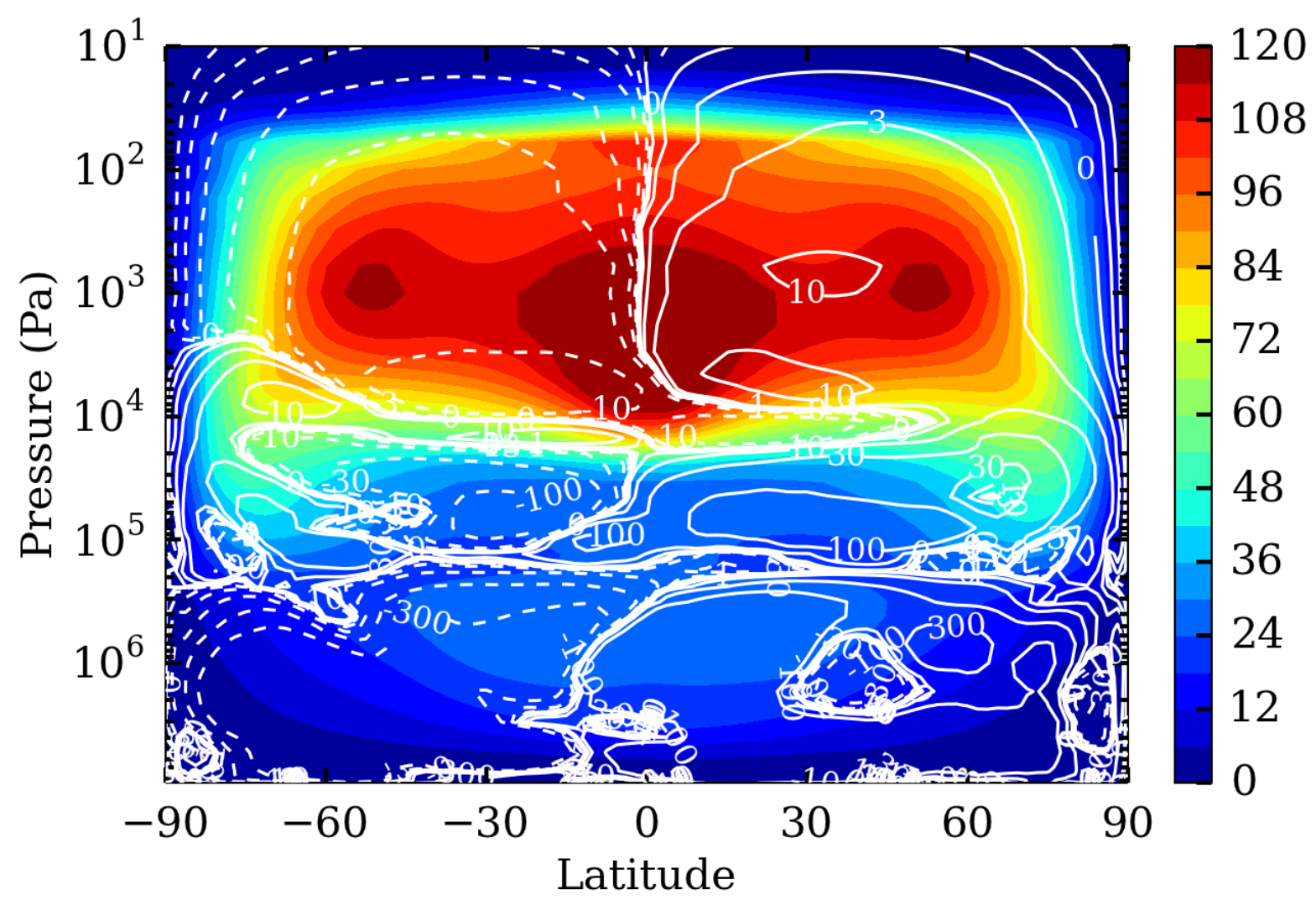


Figure 33. Circulation obtained with the LMD Venus GCM (Lebonnois et al., 2016). Mean zonal wind $\left(\mathrm{m} \mathrm{s}^{-1}\right)$ and stream function (white contours, $10^{9} \mathrm{~kg} \mathrm{~s}^{-1}$ ) averaged over 2 Venus days after 190 Venus days of simulation (simulation with full radiative transfer and topography, started from super-rotation). Reproduced from Lebonnois et al. (2016).

The most recent efforts to develop radiative transfer modules for GCMs have been published by Lee and Richardson (2011) and Mendonca et al. (2015). Lee and Richardson (2011) developed a radiative transfer model based on a multiple-stream, discrete ordinate flux solver. Mendonca et al. (2015) use two different approaches to compute solar and infrared radiative budgets. The solar radiative transfer is based on a two-stream, delta-Eddington approximation, with an adding layer method. For the thermal radiation, an absorptivity/emissivity formulation is used. Both the Lee and Richardson (2011) and Mendonca et al. (2015) radiative schemes were used to calculate solar and atmospheric infrared fluxes with a prescribed temperature profile and to evaluate radiative-convective equilibrium temperatures. The Mendonca et al. (2015) radiative scheme has also been used in the recent Venus GCM published by Mendonca and Read (2016). This model is a direct extension of the simple GCM, developed by Lee et al. $(2005,2010)$ and based on the Met Office Unified Model dynamical core, to compute realistic radiative transfer, together with an improved boundary layer scheme and a more accurate representation of convection with a temperature-dependent specific heat, following Lebonnois et al. (2010). The first results presented by Mendonca \& Read (2016) assumed a fixed distribution of clouds, again following Lebonnois et al. (2010, 2016), although in principal the radiation scheme can handle interactive clouds that vary in both space and time. Their simulations used a spatial resolution of $5^{\circ} \times 5^{\circ}$ in the horizontal and 37 layers in the vertical. The results broadly confirm those obtained by Lebonnois et al. (2010) in forming a reasonably realistic super-rotation at the cloud level, driven by a combination of thermal tides and planetary waves, but only weak zonal winds in the deep atmosphere. They did, however, note some sensitivity of the circulation to parameters such as the assumed surface albedo and deep atmosphere opacity, which affected the static stability in the deep atmosphere and consequent exchanges of angular momentum. Such issues merit further investigation in future studies that may examine in more detail such dynamical and thermal interactions of the deep atmosphere with the surface.

\subsubsection{Main issues in Venus atmospheric modeling}

Success in producing super-rotation in Venus's or Titan's atmospheric GCMs has long-time been a challenge. Though the most recent efforts, basically since the 1990s, have been more successful, realistic simulations of Venus's atmospheric super-rotation remain quite tricky to achieve. The modeled zonal wind field appears to be extremely sensitive to many model parameters. As an example, the impact of the topography on the modeled circulation was discussed in Herrnstein and Dowling (2007), Lee et al. (2007) and Lebonnois et al. (2010). In Herrnstein and Dowling (2007), adding the topography was found to reduce the zonal wind peak velocity, inducing an interhemispheric asymmetry with a slower jet in the northern hemisphere. For Lee et al. (2007), the impact was found to be small, and the asymmetry induced was the opposite, with a stronger northern jet. The simulations in Lebonnois et al. (2010), however, showed an increase in super-rotation when the topography was taken into account, and no significant asymmetry in the zonal wind. The sensitivity to parameters and the difficulty in obtaining robust results can also be illustrated by the sensitivity to initial state, as discussed in 
2398 Kido and Wakata (2008), to resolution, or to the choice of dynamical core, as first illustrated in 2399 Lee and Richardson (2010) who used three different dynamical cores with the same physical 2400 parameterizations and obtained results that were significantly different. 
To demonstrate and explore this sensitivity, an intercomparison effort was conducted within the framework of a working group sponsored by the International Space Science Institute (ISSI) in Bern. Many Venus GCM modelers ran simulations under a common protocol, using the same physical forcing and simple parameterizations to see how robust was the obtained circulation. Temperature forcing was applied using Newtonian cooling, similar to the set-up of Lee et al. (2005). The inter-comparison results were published as part of a book gathering the conclusions of this working group (Lebonnois et al., 2013). The averaged zonal wind fields obtained with the baseline simulation configuration and eight different dynamical cores are presented in Fig. 34. It illustrates the very large diversity of results, with different shapes and magnitudes for the zonal wind maxima. In the same work, sensitivity to resolution, topography, boundary conditions and initial state were also studied, which also resulted in a large diversity among GCMs. This work demonstrates that, under the weak forcing and long timescales characteristic of the atmosphere of Venus, detailed differences in the different formulations of the dynamical core may induce extremely variable responses in the zonal wind field. This is due to the extreme sensitivity of the angular momentum budget. In particular, further studies have investigated the question of angular momentum conservation in the dynamical cores: Lebonnois et al. (2012) showed the performances of the LMD Venus GCM (fair) and of the VenusCAM finite-volume core (poor), while Lee and Richardson (2012) investigated this conservation aspect for their spectral core, demonstrating an excellent degree of conservation. This angular momentum conservation and how it affects the overall budget may be partly responsible for the diversity seen in Figure 34.

The implementation of schemes parameterizing the impact of mesoscale gravity waves on Venus atmospheric circulation is another issue that needs additional investigation. These mesocale gravity waves may be generated in the convective region of the cloud layer, for example, propagating both upwards and downwards and transporting angular momentum. They may also be generated near the surface, through convective activity or as a result of interactions with the topography. In the case of lee waves, the minimum magnitude needed for the surface winds to be able to generate such waves propagating up to the cloud layers has never been investigated, which would certainly be an interesting constraint. These waves can therefore play a role in the acceleration of the circulation below the clouds, and in its deceleration above. A specific parameterization is needed to take their impact into account. Ikeda (2011) made an attempt to apply such a parameterization for gravity waves generated near the surface, showing an improvement in the deep atmosphere super-rotation. Additional efforts are needed to confirm this result, however, to include waves generated in the cloud convective layer, and to thoroughly investigate the role of these small-scale gravity waves on the overall angular momentum budget.

The representation of the polar vortex in the GCMs may also be an issue that needs further investigation. The use of polar filters in grid coordinate dynamical cores can alter the way waves behave, and could also be partly responsible for the apparent sensitivity to resolution. Further studies should investigate this aspect, and explore the polar circulation obtained using new dynamical cores that do not suffer from the polar singularity, such as new cores based on a cubed-sphere grid (e.g. CAM-SE developed at NCAR) or an icosahedral grid (e.g. Dynamico, developed at LMD). Though some GCM simulations exhibit a plausible polar vortex structure (such as Yamamoto and Takahashi (2015), Ando et al. (2015b) and Lebonnois et al. (2016)), the modeling of the cold collar region also needs improvement. To reach this goal, it is certainly 
2447 necessary to represent realistically the coupling between the latitudinally-varying cloud structure, 2448 the cold area and the dynamics of the polar regions, in particular the solar tides. 

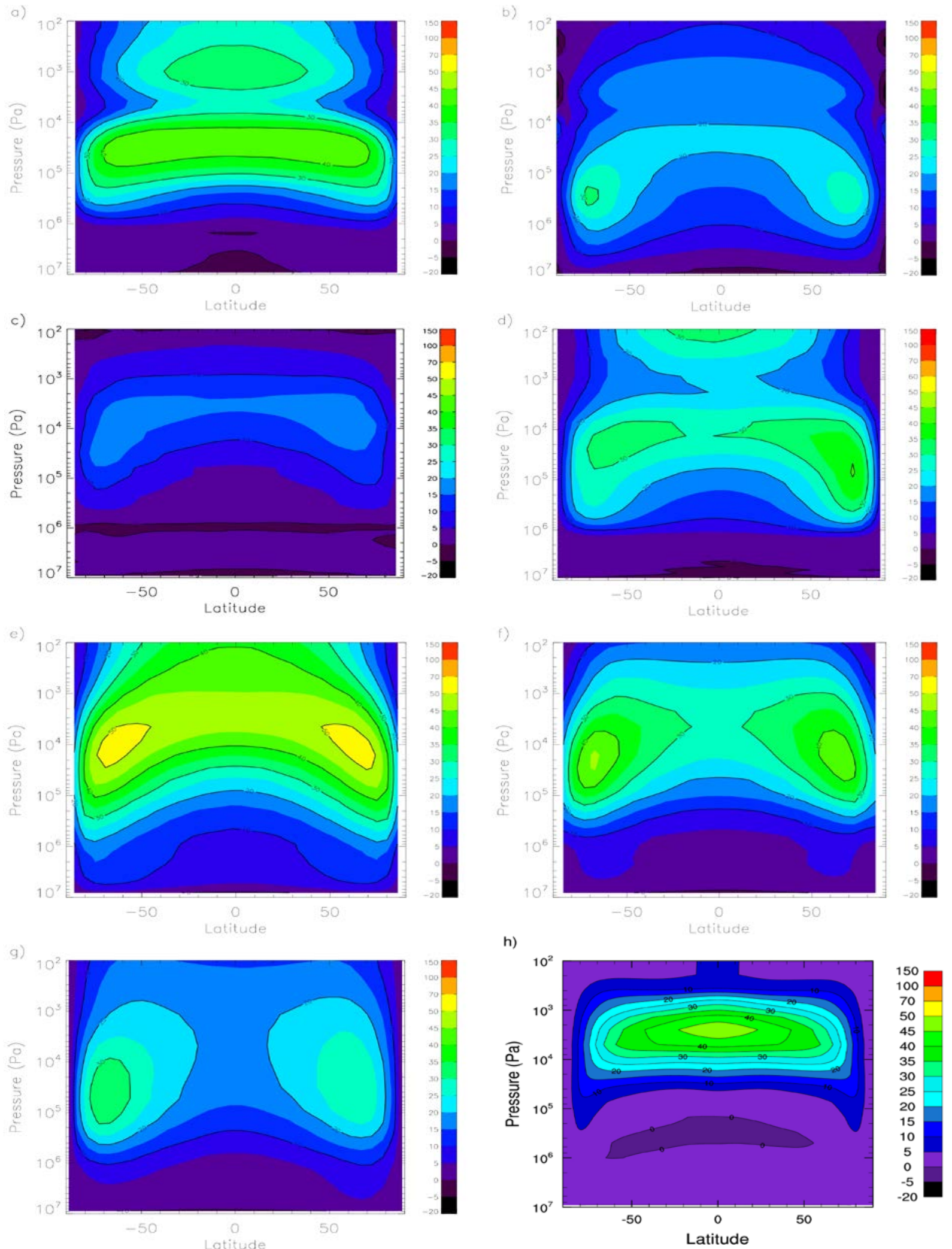

Figure 34. Zonally and temporally averaged zonal wind fields obtained in all the baseline 2452 simulations of the intercomparison exercise done by the working group sponsored by ISSI. Unit 
is $\mathrm{m} / \mathrm{s}$. The first column is spectral models: (a) CCSR (Yamamoto and Takahashi), (c) Open University (Lewis et al.), (e) Lee and Richardson (2010) spectral core. The second column is

\subsubsection{Taking into account non-LTE processes}

The GCMs described above are limited to altitudes below around $100 \mathrm{~km}$, because additional processes need to be taken into account in the upper atmosphere. At pressures below $0.1 \mathrm{~Pa}$ in a $\mathrm{CO}_{2}$ atmosphere, the Local Thermal Equilibrium (LTE) assumption is not valid anymore, because the molecular emissions do not follow a Planckian source function at the local kinetic temperature, but reflect a diversity of radiative, collisional and chemical processes which are specific to each molecular species and vibrational transition. The heating and the cooling of the atmosphere are therefore controlled by non-LTE radiative transfer processes, and a specific code is required for their description and interpretation. Detailed calculations of the non-LTE radiative transfer have been performed for the Venus atmosphere by Roldan et al. (2000) and LópezValverde et al. (2007). Above roughly $120 \mathrm{~km}$, in addition to non-LTE radiative transfer, the thermal structure is also controlled by the absorption of Extreme UV radiation, and thermal conduction. The atmosphere is not well mixed anymore and molecular diffusion has an impact on the composition of the atmosphere. The composition is also affected by an active photochemistry, which must be taken into account to understand the thermal structure and the dynamics.

To model these upper atmospheric regions, two different approaches have been developed, in particular for the study of the Martian atmosphere: either thermospheric general circulation models (TGCM, e.g. Bougher and Dickinson, 1988; Bougher et al., 1990) modeling the atmospheric circulation above a given altitude level, or vertically extended GCMs that include these additional parameterizations, such as the LMD Mars GCM (e.g. Angelats i Coll et al., 2005; Gonzalez-Galindo et al., 2009). For Venus, Bougher et al. $(1986,1988)$ started the development of a Venus Thermospheric GCM (finite-difference grid), recently updated in Brecht et al. (2011) and Brecht and Bougher (2012), that includes the non-LTE heating and cooling rates, with look-up tables for the solar heating rates and a parameterized scheme for the cooling rates which implements a line-by-line model for $15-\mu \mathrm{m} \mathrm{CO}$ cooling rates taken from Roldan et al. (2000). This VTGCM covers altitudes from $70 \mathrm{~km}$ to $200 \mathrm{~km}$ on the nightside and $300 \mathrm{~km}$ on the dayside, with a horizontal resolution of $5^{\circ} \times 5^{\circ}$. Adjusting some of the model parameters, the atmospheric circulation modeled for mean conditions has been compared to the $\mathrm{O}_{2}$ and NO nightglows observed from Venus-Express instruments (Brecht et al., 2011). The modeled circulation was composed of the superposition of two regimes: the super-rotation at lower altitudes and a sub-solar to anti-solar (SS-AS) thermospheric circulation. A parameterized drag was used to relax the zonal wind towards a specified zonal wind profile, to approximate the super-rotating component. The thermal structure obtained in Brecht and Bougher (2012) reproduces some of the observed features, with alternating minimum and maximum temperatures between 80 and $150 \mathrm{~km}$ on the dayside and a warm region around midnight, close to $105 \mathrm{~km}$, 
corresponding to nightside downwelling of the SS-AS circulation. However, when properly convolved to be compared with the temperatures obtained for the $\mathrm{CO}_{2}$ non-LTE emissions from VIRTIS-H (Peralta et al. 2016), the VTGCM obtain temperatures systematically warmer than the observations (by 20-25K at the sub-solar point). Using the same VTGCM, Zalucha et al. (2013) included a gravity-wave parameterization to study the impact of the angular momentum transport of these small-scale waves generated in the cloud layer (i.e. at the base of the VTGCM) on the zonal wind field. However, this parameterization was not successful in producing sufficient drag to get agreement with available observations.

Hoshino et al. (2012) also developed a VTGCM based on a finite-difference dynamical core, covering from 80 to roughly $180 \mathrm{~km}$ altitude, in which the SS-AS circulation dominated the winds above $90 \mathrm{~km}$. The non-LTE processes were parameterized as in Bougher et al. (1986). The zonal wind was assumed to be $0 \mathrm{~m} \mathrm{~s}^{-1}$ at the lower boundary of the model, and thermal tides and planetary scale waves were forced at this lower boundary to study how these waves propagate into the upper atmosphere and affect the circulation, in particular the effect of the Kelvin wave on the $\mathrm{O}_{2}$ nightglow. Hoshino et al. (2013) added a gravity-wave parameterization and studied the differences in the modeled circulation between dayside and nightside. The dynamical core is now spectral with T21 horizontal resolution, and the non-LTE processes are based on Roldan et al. (2000). This model shows that, with a solid-body rotation zonal wind of $40 \mathrm{~m} \mathrm{~s}^{-1}$ over the equator at the lower boundary $(\sim 80 \mathrm{~km})$, the gravity waves transfer angular momentum above $100 \mathrm{~km}$, inducing a layer in the $95-130 \mathrm{~km}$ region where super-rotation strongly contributes to the zonal circulation again (Figure 35).
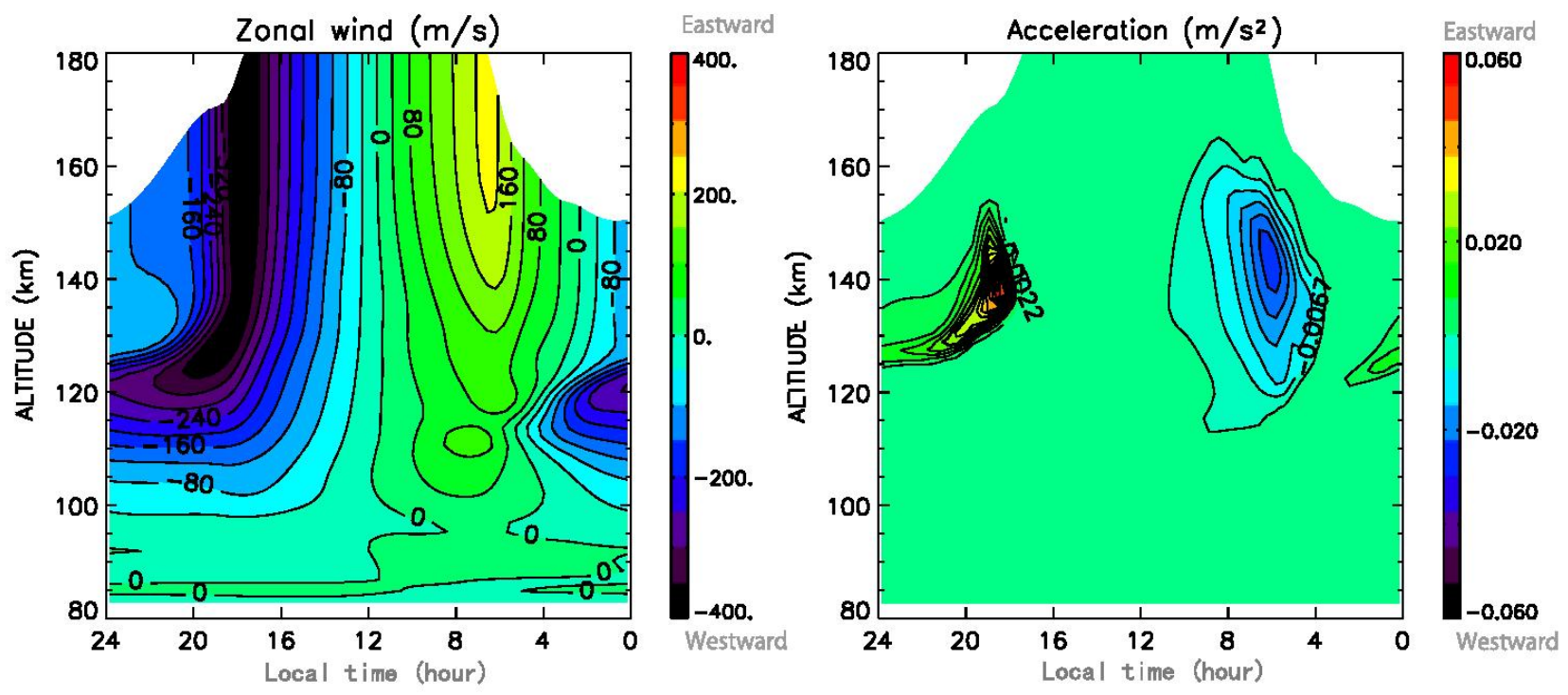

2521

2522

2523

2524

2525

2526

2527

2528

Figure 35. Simulations from the Hoshino et al. (2013) VTGCM, showing the local time-height distribution of (left) the zonal wind in $\mathrm{m} \mathrm{s}^{-1}$ and (right) the wave drag acceleration (in $\mathrm{m} \mathrm{s}^{-2}$ ) calculated from the gravity-wave parameterization scheme, at the equator and with a lowerboundary zonal wind fixed at solid-body rotation with a velocity of $-40 \mathrm{~m} \mathrm{~s}^{-1}$ at the equator. Positive and negative values of the zonal wind distribution indicate eastward and westward winds, respectively. Reproduced from Hoshino et al. (2013). 
To improve the modeling of the interaction between the deep and upper regions of the atmosphere, another type of development has been to extend GCMs that cover the deep atmosphere to take into account the upper atmospheric processes and to raise their upper boundary into the thermosphere. This development was conducted with the LMD Venus GCM (Gilli et al., 2017). The model used a 1-parameter analytical formula to reproduce the solar heating rates (based again on Roldan et al., 2000), and a simplified non-LTE model for the 15$\mu \mathrm{m}$ cooling, following the methodology developed for the LMD Mars GCM (Gonzalez-Galindo et al., 2009). It was assumed that the net absorption depends mainly upon the density of the atmosphere, and to a smaller degree on the solar zenith angle, thermal structure and atomic oxygen abundance. Similarly to other works, EUV absorption was parameterized assuming an efficiency of 20-22 \%, and the variation of the UV solar flux with the solar cycle was taken into account. This model was coupled with a photochemical model, allowing the composition to be consistently computed, which is especially needed for the atomic oxygen. It also included a gravity wave parameterization scheme, based on a stochastic upward emission of gravity waves by the convective cloud layer. The predicted temperature structure was consistent with observations and with the other models, though quantitative agreement with the observed minimum and maximum temperature values (and their altitudes) remains to be improved. As in Hoshino et al. (2013), the gravity waves have an important role in controlling the circulation in the $90-120 \mathrm{~km}$ altitude region, and additional work is needed to study their generation and characteristics.

\section{Discussion and Conclusions}

We summarize and discuss in this final section the observations and theoretical and modeling efforts required to explain the yet to be answered questions of Venus's atmospheric dynamics:

- Measurement of dynamical variables. The precise wind field retrieval below the upper clouds (from the surface to $60 \mathrm{~km}$ ) as a function of location (longitude and latitude) and local time is an essential ingredient required to derive the vertical distribution of the angular momentum, momentum transfers and super-rotation origin. An accurate measurement of the 3D wind speeds (on the order of $5 \mathrm{~m} \mathrm{~s}^{-1}$ or less), in areas with complex turbulent cloud patterns and waves, will allow the characterization of eddy motions necessary for constraining GCMs. Meridional motions need to be better constrained at different altitude levels to test the presence and properties of Hadley circulations and the transport of heat and momentum by thermal tides at the upper cloud level. Simultaneous retrievals of wind speeds and other dynamical variables, such as temperature and pressure, will allow a better understanding of the "quasi-cyclostrophic" balance, the presence or not of jets, and to study their stability. All this will require further in situ exploration by descending probes and balloons, supported by remote sensing studies from orbit.

- Wave studies. Major classes of atmospheric waves seen in the Earth's atmosphere, such as gravity, Kelvin, Rossby and tidal waves, also exist in the Venus atmosphere. The waves are expected to play crucial roles in the general circulation of the atmosphere via their contributions to momentum transport, both in altitude and latitude. The 
characteristics or existence of theses waves are thought to be strongly related to the inertial forces associated with the super-rotation. Strong thermal tides are excited due to the absorption of sunlight by the thick cloud layer covering the whole surface. The sources of gravity, Rossby and Kelvin waves, the origins of the observed frequencies and wavelengths of those waves, and the associated momentum transports are poorly understood. Observations of the waves below the clouds are extremely scarce, and numerical modeling of the waves is not robust enough, due to uncertainties in the background atmospheric state. Calculation of the fluxes of energy and momentum from the wave dispersion relationships and amplitudes will represent an important advance in their role on dynamics.

- Polar Vortices. With its highly elliptical, low inclination orbit, Venus Express has accomplished a formidable task in characterizing the southern hemisphere's polar vortex dynamics, providing new spatially and temporally resolved measurements of winds, cloud heights and temperatures. Since its first detection early in the mission, it became clear that variability is a major characteristic of the polar vortex. Despite accurate characterization of its 3D wind and temperature structure, outstanding questions remain about polar vortex dynamics. Why is the vortex morphology similar at both the upper and lower cloud levels, despite the vertical shear of the zonal wind? Is downwelling at the pole, presumably from the descending branch of the Hadley cell, causing the variability of the vortex morphology and the peculiar motion of its centroid of rotation, or do heat sources and sinks below the clouds control this variability? How are vortex dynamics and cloud height connected? Is the cold collar acting to contain the polar vortex and isolate it from interaction with the atmosphere at mid and low latitudes, or is it part of a global hemispheric vortex? Are occasional wind jets at mid-latitudes the main cause of barotropic instability at the Venus cloud tops, or is there a persistent source of barotropic instability at the vortex eyewall? Why is there no apparent connection between the thermal emission features and the vorticity distribution? How do the solar migrating atmospheric tides interact with the mean background flow and what is their contribution to the angular momentum budget of the polar region? Quantitatively answering these questions will require moving in multiple directions. On the observational side, improved measurements of temperatures, cloud height and winds are required, in particular more precise zonal and meridional winds. This will help determine the meridional wind direction at latitudes poleward of $70^{\circ}$ in order to constrain the extent of the Hadley cell, and also to reduce wind divergence uncertainty in order to better constrain cloud top sources and sinks. On the modeling side, spatially resolving the polar vortex morphology, accurately reproducing its dynamics and polar wave motions and its connection with the flow at lower latitudes is required.

- Nature of the Super-rotation. One of the most compelling outstanding dynamical issues in Venus's atmosphere continues to lie in accounting for the origin and maintenance of its extreme super-rotation. As reviewed here, the basic GRW scenario seems convincingly capable in principle of sustaining such a super-rotation by extracting angular momentum from the underlying planet. But the precise nature and scope of the eddy processes acting within such a scenario remain uncertain. Numerical model simulations with reasonably accurate and realistic representations of radiative forcing and dynamics have recently 
proved capable of capturing many aspects of the observed super-rotation, especially in the middle and upper atmosphere, despite the unexpectedly strong sensitivity of the simulated circulation to the numerical formulation and spatial resolution. Such model simulations have illustrated key roles for the thermal tides and equatorial planetary waves in the transport of angular momentum in the vicinity of the main cloud decks. But many aspects of the circulation in the deep atmosphere, at altitudes below $40 \mathrm{~km}$ where the bulk of the atmospheric angular momentum apparently resides, are poorly constrained by observations. It is far from clear, for example, how eddy processes penetrate into the deep atmosphere and how they interact with the planetary surface. The role of small-scale internal gravity waves is particularly uncertain. Although there continues to be scope for improving the existing models in various ways, through enhancing spatial resolution, numerical formulations and parameterizations, there is a particularly urgent need for more detailed observations of the deep atmosphere that can match the coverage obtained by missions such as Venus Express in the middle atmosphere.

- General Circulation Models. The GCMs that have been developed to study the dynamics of Venus's atmosphere have made much progress in recent years. They now include detailed radiative transfer modules that allow simulations to reproduce the main features of the thermal structure. The balance of angular momentum accounting for the main features of the zonal wind field involves transport by the mean meridional circulation, planetary-scale waves and thermal tides. Although a realistic super-rotation in the cloud region is now obtained by most models, several issues remain to be investigated to fit the details of the observed wind field. First, the robustness of the simulations need to be confirmed through inter-comparison between several GCMs, since the modeled atmospheric circulation is very sensitive to the technical details of the dynamical core. Conservation of angular momentum is one important point to check. Then, the role of subgrid-scale processes in the angular momentum budget, especially small-scale gravity waves, needs to be assessed. Validated parameterizations of these processes are crucial to reach this goal. Despite these remaining validations and improvements, the Venus GCM simulations may now be realistic enough to start data assimilation projects, taking advantage of the huge datasets gathered by the Venus Express and Akatsuki missions. This would help to further improve our understanding of Venus's atmospheric dynamics.

\section{Acknowledgements}

ASL was supported by the Spanish MICIIN AYA2015-65041-P (MINECO/FEDER, UE), Grupos Gobierno Vasco IT765-013. We appreciate the careful and detailed comments provided by two anonymous reviewers. PLR acknowledges support from the UK Science and Technology Facilities Council under grants ST//001948/1 and ST/K00106X/1.

\section{References}


M.J. Alexander, A mechanism for the Venus thermospheric super-rotation. Geophys. Res. Lett. 19, 2207-2210 (1992).

F.,Altieri, A. Migliorini, L. Zasova, A. Shakun, G. Piccioni, and G. Bellucci Modeling VIRTIS/VEX O2(a1 $\Delta \mathrm{g})$ nightglow profiles affected by the propagation of gravity waves in the Venus upper mesosphere, J. Geophys. Res.Planets, 119, 2300-2316, (2014)

H. Ando, T. Imamura, T. Tsuda, S. Tellmann, M. Pätzold, B. Häusler, Vertical wavenumber spectra of gravity waves in the Venus atmosphere obtained from Venus Express radio occultation data: Evidence for saturation. J. Atmos. Sci., 72, 2318-2329 (2015)

H. Ando, N. Sugimoto, M. Takagi, H. Kashimura, T. Imamura, Y. Matsuda, The puzzling Venusian polar atmospheric structure reproduced by a general circulation model, Nature Comm., 1:101038 (2016)

D.G. Andrews, J.R. Holton, C.B. Leovy, Middle Atmosphere Dynamics (Academic Press, New York, 1987), 489 pp.

M. Angelats-i-Coll, F. Forget, M. A. López-Valverde, F. Gonzalez-Galindo, The first Mars Thermospheric general circulation model: the Martian atmosphere from the ground to $240 \mathrm{~km}$. Geophys. Res. Lett. 32(4), L05201 (2005). doi:10.1029/2004GL021368

J. Apt, R.A. Brown, R.M. Goody, The character of the thermal emission from Venus. J. Geophys. Res. 85, 7934-7940 (1980).

N. Baker, C. B. Leovy, Zonal winds near Venus' cloud top level: A model study of the interaction between the zonal mean circulation and the semidiurnal tide. Icarus 69, 202-220 (1987).

R.D. Baker, G. Schubert, P.W. Jones, Cloud-level penetrative compressible convection in the Venus atmosphere, J. Atmos. Sci. 55, 3-18 (1998).

R.D. Baker, G. Schubert, P.W. Jones, Convectively generated internal gravity waves in the lower atmosphere of Venus. Part I: No wind shear. J. Atmos. Sci. 57, 184-199 (2000a).

R.D. Baker, G. Schubert, P.W. Jones, Convectively generated internal gravity waves in the lower atmosphere of Venus. Part II: Mean wind shear and wave-mean flow interaction, J. Atmos. Sci. 57, 200-215 (2000b).

M. P. Baldwin, L. J. Gray, T. J. Dunkerton, K. Hamilton, P. H. Haynes, W. J. Randel, J. R. Holton, M. J. Alexander, I. Hirota, T. Horinouchi, D. B. A. Jones, J. S. Kinnersley, C. Marquardt, K. Sato, and M. Takahashi, The Quasi-Biennial Oscillation, Rev. Geophys., 39, 179229 (2001). 
J.K. Barstow, C.C.C. Tsang, C.F. Wilson, P.G.J. Irwin, F.W. Taylor, K. McGouldrick, P. Drossart, G. Piccioni, S. Tellman, Models of the global cloud structure on Venus derived from Venus Express observations, Icarus 217, 542-560 (2012).

M. J. S. Belton, G. R. Smith, G. Schubert, A. Del Genio, Cloud Patterns, Waves and Convection in the Venus Atmosphere, J. Atmos. Sci., 33, 1394 - 1417 (1990).

M.J.S. Belton, et al., Images from Galileo of the Venus cloud deck, Science 253, 1531-1536 (1991).

M. J. S. Belton, , K. P. Klaasen, M. C. Clary, J. L. Anderson, C. D. Anger, M. H. Carr, C. R. Chapman, et al. 1992, The Galileo Solid-State Imaging Experiment, Space Science Reviews 60: 413-455 (1992).

J. L. Bertaux, I. V. Khatunsev, A. Hauchecorne, W.J. Markiewicz, E. Marq, S. Lebonnois, M. Patsaeva, A. Turin, A. Fedorova, Influence of Venus topography on the zonal wind and UV albedo at cloud top level: The role of stationary gravity waves, J. Geophys. Res. Planets, 121, doi:10.1002/2015JE004958

J. E. Blamont, R. E. Young, A. Seiff, B. Ragent, R. Sagdeev, V. M. Linkin, V. V. Kerzhanovich, A. P. Ingersoll, D. Crisp, L. S. Elson, R. A. Preston, G. S. Golytsin, V. N. Ivanov, Implications of the VEGA Balloon Results for Venus Atmospheric Dynamics, Science 231, 1422 - 1425 (1986)

S. W. Bougher, R. E. Dickinson, Mars mesosphere and thermosphere. I - Global mean heat budget and thermal structure. J. of Geophys. Res. 93, 7325-7337 (1988). doi:10.1029/JA093iA07p07325

S. W. Bougher, R. E. Dickinson, E. C. Ridley, R. G. Roble, A. F. Nagy, T. E. Cravens, Venus mesosphere and thermosphere: II. Global circulation, temperature, and density variations. Icarus 68, 284-312 (1986). doi:10.1016/0019-1035(86)90025-4

S. W. Bougher, R. G. E. Roble, R. E. Dickinson, E. C. Ridley, Venus mesosphere and thermosphere: III. Three-dimensional general circulation with coupled dynamics and composition. Icarus 73, 545-573 (1988). doi:10.1016/0019-1035(88)90064-4

S. W. Bougher, R. G. Roble, E. C. Ridley, R. E. Dickinson, The Mars thermosphere. II - General circulation with coupled dynamics and composition. J. of Geophys. Res. 95, 14811-14827 (1990). doi:10.1029/JB095iB09p14811

S. W. Bougher, M. J. Alexander, H. G. Mayer, Upper atmosphere dynamics: global circulation and gravity waves, in Venus II, eds. S. W. Bougher, D. M. Hunten, R. J. Phillips (University of Arizona Press, Tucson, 1997) pp. 259-29.

A. S. Brecht, S. W. Bougher, J.-C. Gérard, C. D. Parkinson, S. Rafkin, B. Foster (2011), Understanding the variability of nightside temperatures, NO UV and $\mathrm{O}_{2}$ IR nightglow emissions 
2756

2757

2758

2759

2760

2761

2762

2763

2764

2765

2766

2767

2768

2769

2770

2771

2772

2773

2774

2775

2776

2777

2778

2779

2780

2781

2782

2783

2784

2785

2786

2787

2788

2789

2790

2791

2792

2793

2794

2795

2796

2797

2798

2799

2800

2801

in the Venus upper atmosphere. J. of Geophys. Res. (Planets) 116, E08004 (2011). doi:10.1029/2010JE003770

A. S. Brecht, S. W. Bougher, Dayside thermal structure of Venus' upper atmosphere characterized by a global model. J. of Geophys. Res. 117, E08002 (2012). doi:10.1029/2012JE004079

L. Colin, Basic facts about Venus, in Venus I, eds. D. M. Hunten, L. Colin, T. M. Donahue, V. I. Moroz, (University of Arizona Press, Tucson, 1983) pp. 10-26.

C. C. Counselman III, S. A. Gourevich, R. W. King, G. B. Loriot, Zonal and meridional circulation of the lower atmosphere of Venus determined by radio interferometriy. J. Geophys. Res., 85: 8026-8030 (1980)

C. Covey, G. Schubert, Planetary-scale waves in the Venus atmosphere, J. Atmos. Sci. 39, $2397-$ 2413 (1982).

A. D. Del Genio, W.B. Rossow, Planetary-scale waves and the cyclic nature of cloud top dynamics on Venus, J. Atmos. Sci. 47, 293-318 (1990).

A. R. Dobrovolkis, D. J. Diner, Barotropic instability with divergence: theory and applications to Venus, J. Atmos. Sci., 47, 1578-1588 (1990).

P. Drossart, F. Montmessin, The legacy of Venus Express: highlights from the first European planetary mission to Venus, Astron. Astrophys. Rev., 23:5 (2015).

L. S. Elson, Barotropic instability in the upper atmosphere of Venus. Geophys. Res. Lett., 5(7), 603-605 (1978).

L. S. Elson, Wave instability in the polar region of Venus. J. Atmos. Sci., 39, 2356-2362 (1982)

L.W. Esposito, Ultraviolet contrasts and the absorbers near the Venus cloud tops. J. Geophys. Res. 85, 8151-8157 (1980).

L. W.Esposito, J. L. Bertaux, V Krasnopolsky, V. I. Moroz, and L. V. Zasova. Chemistry of Lower Atmosphere and Clouds eds. S. W. Bougher, D. M. Hunten, R. J. Phillips, (University of Arizona Press, Tucson, 1997) pp 415-458

V. Eymet, R. Fournier, J.-L. Dufresne, S. Lebonnois, F. Hourdin, M. A. Bullock, Net-exchange parameterization of the thermal infrared radiative transfer in Venus' atmosphere. J. of Geophys. Res. 114, E11008 (2009). doi:10.1029/2008JE003276

A. Fedorova, E. Marcq, M. Luginin, O. Korablev, J.-L. Bertaux, F. Montmessin, Variations of water vapor and cloud top altitude in the Venus' mesosphere from SPICAV/VEx observations, Icarus 275, 143-162 (2016) 
2802

2803

2804

2805

2806

2807

2808

2809

2810

2811

2812

2813

2814

2815

2816

2817

2818

2819

2820

2821

2822

2823

2824

2825

2826

2827

2828

2829

2830

2831

2832

2833

2834

2835

2836

2837

2838

2839

2840

2841

2842

2843

2844

2845

2846

2847

S.B. Fels, R.S. Lindzen, The interaction of thermally excited gravity waves with mean flows. Geophys. Fluid Dyn. 6, 149-191 (1974).

F. M. Flasar, K. H. Baines, M. K. Bird, T. Tokano and R. A. West, Atmospheric dynamics and meteorology, in Titan from Cassini-Huygens (eds. R. H. Brown, J.-P. Lebreton, J. Hunter-Waite), (Springer, Netherlands, 2009), pp.323-352.

T. Fukuhara, M. Futaguchi, G. L. Hashimoto, T. Horinouchi, T. Imamura, N. Iwagaimi, T. Kouyama, S. Murakami, M. Nakamura, K. Ogohara, M. Sato, T. M. Sato, M. Suzuki, M. Taguchi, S. Takagi, M. Ueno, S. Watanabe, M. Yamada, A. Yamazaki, Large stationary gravity wave in the atmosphere of Venus, Nature Geoscience 10, 85-88 (2017)

I. G. Garate-Lopez, R. Hueso, A. Sánchez-Lavega, J. Peralta, G. Piccioni, P. Drossart, A chaotic permanent vortex in Venus' southern pole, Nature Geoscience 6, 254-257 (2013).

I. G. Garate-Lopez, A. Garcia-Muñoz, R. Hueso, A. Sanchez-Lavega, Three-dimensional Thermal Structure of the South Polar Vortex of Venus, Icarus 245, 16-31 ( 2015).

R. F. Garcia, P. Drossart, G. Piccioni, M. López-Valverde, and G. Occhipinti, Gravity Waves in the Upper Atmosphere of Venus Revealed by CO 2 Nonlocal Thermodynamic Equilibrium Emissions, J. Geophys. Res. 114, E00B32 (2009) doi:10.1029/2008JE003073.

A. García Muñoz, P. Wolkenberg, A. Sánchez-Lavega, R. Hueso, I. Garate-Lopez, A model of scattered termal radiation for Venus from 3 to $5 \mu \mathrm{m}$, Planetary and Space Sciences 81, 65-73 (2013).

P. J. Gierasch, Meridional circulation and the maintenance of the Venus atmospheric rotation. J. Atmos. Sci., 32, 1038-1044 (1975)

P. J. Gierasch, Waves in the atmosphere of Venus, Nature 328, 510-512 (1987).

P. J. Gierasch, R. M. Goody, R. E. Young, D. Crisp, C. Edwards, R. Kahn, D. McCleese, D. Rider, A. Del Genio, R. Greeley, A. Hou, C. B. Leovy, N. Newman. The General Circulation of the Venus Atmosphere: An Assesment, in Venus II, eds. S. W. Bougher, D. M. Hunten, R. J. Phillips, (University of Arizona Press, Tucson, 1997) pp. 459-500.

G. Gilli, S. Lebonnois, F. González-Galindo, M. A. López-Valverde, A. Stolzenbach, F. Lefèvre, J.-Y. Chaufray and F. Lott. Thermal structure of the upper atmosphere of Venus simulated by a ground-to-thermosphere GCM. Icarus 281, 55-72 (2017).

G. Gilli, , M. a. López-Valverde, P. Drossart, G. Piccioni, S. Erard, A. Cardesín Moinelo, Limb Observations of CO 2 and CO Non-LTE Emissions in the Venus Atmosphere by VIRTIS/Venus Express, J. Geophys. Res. 114, E00B29 (2009) doi:10.1029/2008JE003112.

F. Gonzalez-Galindo, F. Forget, M. A. López-Valverde, M. Angelats-i-Coll, A ground-toexosphere Martian general circulation model: 1. Seasonal, diurnal, and solar cycle variation of 
thermospheric temperatures. J. of Geophys. Res. (Planets) 114, E04001 (2009). doi:10.1029/2008JE003246

D. Grassi, A. Migliorini, L. Montabone, S. Lebonnois, A. Cardesin-Moinelo, G. Piccioni, P. Drossart, L. V. Zasova, Thermal structure of Venusian nighttime mesosphere as observed by VIRTIS-Venus Express. J Geophys. Res., 115, E09007 (2010) doi:10.1029/2009JE003553.

D. Grassi, D. et al., The Venus Nighttime Atmosphere as Observed by the VIRTIS-M Instrument. Average Fields from the Complete Infrared Data Set J. Geophys. Res.- Planets 837849 (2014) doi:10.1002/2013JE004586.

R. Greeley, K. Bender, P. E. Thomas, G. Schubert, D. Limonadi, C. M. Weitz,. Wind-Related Features and Processed on Venus, Icarus 115, 399-420 (1995)

S. D. Griffiths, The nonlinear evolution of zonally symmetric equatorial inertial instability, J. Fluid Mech. 474, 245-273 (2003).

J. Gula, R. Plougonven, V. Zeitlin, Ageostrophic instabilities of fronts in a channel in a stratified rotating fluid, J. Fluid Mech., 627, 485-507 (2009) doi:10.1017/S0022112009006508

I. M. Held and A. Y. Hou, Nonlinear axially symmetric circulations in a nearly inviscid atmosphere. J. Atmos. Sci., 37, 515-533 (1980).

A. Herrnstein, T. E. Dowling, Effect of topography on the spin-up of a Venus atmospheric model. J. of Geophys. Res. 112, E04S08 (2007). doi:10.1029/2006JE002804

R. Hide, Dynamics of the atmospheres of the major planets, with an appendix on the viscous boundary layer at the rigid bounding surface of an electrically conducting rotating fluid in the presences of a magnetic field, J. Atmos. Sci., 26, 841-853 (1969)

D.P. Hinson, J.M. Jenkins, Magellan radio occultation measurements of atmospheric waves on Venus. Icarus 114, 310-327 (1995).

J. R. Holton, An Introduction to Dynamic Meteorology, (Academic Press, Netherlands), (2004).

J. L. Hollingsworth, R. E. Young, G. Schubert, C. Covey, A. S. Grossman, A Simple-physics Global Circulation Model for Venus: Sensitivity assessments of atmospheric super-rotation. Geophys. Res. Lett. 34, L05202 (2007). doi:10.1029/2006GL028567

N. Hoshino, H. Fujiwara, M. Takagi, Y. Takahashi, Y. Kasaba, Characteristics of planetary-scale waves simulated by a new Venusian mesosphere and thermosphere general circulation model, Icarus 217, 818-830 (2012). doi:10.1016/j.icarus.2011.06.039

N. Hoshino, H. Fujiwara, M. Takagi, Y. Kasaba, Effects of gravity waves on the day-night difference of the general circulation in the Venusian lower thermosphere. J. of Geophys. Res. (Planets) 118, 2004-2015 (2013). doi:10.1002/jgre.20154 
A.Y. Hou, Axisymmetric circulations forced by heat and momentum sources - a simple model

A.Y. Hou, B.F. Farrell, Super-rotation induced by critical-level absorption of gravity waves on Venus: An assessment. J. Atmos. Sci. 44, 1049-1061 (1987).

B. L. Hua, D. W. Moore, S. Le Gentil, Inertial nonlinear equilibration of equatorial flows, J. Fluid Mech., 331, 345-371 (1997)

R. Hueso, A. Sánchez-Lavega, G. Piccioni, P. Drossart, J. C. Gérard, I. Khatuntsev, L. Zasova, A. Migliorini, Morphology and Dynamics of Venus Oxygen Airglow from Venus Express /VIRTIS observations, J. Geophys. Res.-Planets 113, E00B02, doi:10.1029/2008JE003081 (2008).

R. Hueso, J. Peralta, A. Sánchez-Lavega, Assessing the Long-Term Variability of Venus Winds at Cloud Level from VRTIS-Venus Express, Icarus 217, 585-598 (2012)

R. Hueso, J. Peralta, I. Garate-Lopez, T. V. Bandos, A. Sánchez-Lavega, Six years of Venus winds at the upper cloud level from UV, visible and near infrared observations from VIRTIS on Venus Express, Planet. Space Sci., in press (2015).

S. Iga, Y. Matsuda, Shear instability in a shallow water model with implications for the Venus atmosphere. J. Atmos. Sci. 62, 2514-2527 (2005).

N.I. Ignatiev, D.V. Titov, G. Piccioni, P. Drossart, W.J. M arkiewicz, V. Cottini, Th. Roatsch, M. Almeida, N. Manoel, Altimetry of the Venus cloud tops from the Venus Express observations, J. Geophys. Res. 114, E00B43 (2009)

K. Ikeda, Development of radiative transfer model for Venus atmosphere and simulation of super-rotation using a general circulation model. Ph.D. dissertation (The University of Tokyo, 2011).

K. Ikeda, M. Yamamoto, M. Takahashi, Super-rotation of the Venus atmosphere simulated by an Atmospheric General Circulation Model. IUGG/IAMAS Meeting, July 2-13, Perugia, Italy (2007).

T. Imamura, Momentum balance of the Venusian midlatitude mesosphere. J. Geophys. Res. 102, 6615-6620 (1997).

T. Imamura, T. Horinouchi, T. Dunkerton, The lateral transport of zonal momentum due to Kelvin waves in a meridional circulation, J. Atmos. Sci. 61, 1966-1975 (2004).

T. Imamura, Meridional propagation of planetary-scale waves in vertical shear: Implication for the Venus atmosphere. J. Atmos. Sci. 63, 1623-1636 (2006). 
T. Imamura, T. Higuchi, Y. Maejima, M. Takagi, N. Sugimoto, K. Ikeda, H. Ando, Inverse J. M. Jenkins, P. J. Steffes, D. Hinson, J. Twicken, L. Tyler, Radio Occultation Studies of Venus’ Atmosphere with the Magellan Spacecraft, Icarus 110, 79-94 (1994).

E. Kalnay de Rivas, Further numerical calculations of the circulation of the atmosphere of Venus. J. of Atm. Sci. 32, 1017-1024 (1975). doi: 10.1175/1520-0469(1975)032

I.V. Khatuntsev, M.V. Patsaeva, D.V. Titov, N.I. Ignatiev, A.V. Turin, S.S. Limaye, W.J. Markiewicz, M. Almeida, Th. Roatsch, R. Moissl, Cloud level winds from the Venus Express Monitoring Camera imaging, Icarus 226, 140-158 (2013)

V. V. Kerzhanovich and M. Ya. Marov, The atmospheric dynamics of Venus according to Doppler measurements by the Venera entry probes, in Venus I, edts. D. M. Hunten, L. Colin, T. M. Donahue, V. I. Moroz, (University of Arizona Press, Tucson, 1983) pp. 766-778.

V. V. Kerzhanovich, S. S. Limaye, Circulation of the atmosphere from the surface to $100 \mathrm{~km}$, Avd. Space Res. 5, 59-83 (1985)

A. Kido, Y. Wakata, Multiple equilibrium states appear in a Venus-like Atmospheric General Circulation Model. J. of the Met. Soc. of Japan 86, 969-979 (2008). doi:10.2151/jmsj.86.969

A. J. Kliore, I. R. Patel, Thermal Structure of the Atmosphere of Venus from Pioneer Venus Radio Occultations, Icarus 52, 320-334 (1982). doi:10.1029/JA085iA13p07957.

A. J. Kliore, V. I. Moroz, G. M. Keating (Editors), The Venus International Reference Atmosphere. Adv. Space Res. 5, 305 pp. (1985).

T. Kouyama, T. Imamura, M. Nakamura, T. Satoh, Y. Futaana, Horizontal structure of planetaryscale waves at the cloud top of Venus deduced from Galileo SSI images with an improved cloudtracking technique. Planet Space Sci. 60, 207-216 (2012). doi: 10.1016/j.pss/2011.08.008

T. Kouyama, T. Imamura, M. Nakamura, T. Satoh, Y. Futaana, Long-term variation in the cloudtracked zonal velocities at the cloud top of Venus deduced from Venus Express VMC images, J. Geophys. Res. Planets 118, 37-46 (2013) DOI:10.1029/2011JE004013

T. Kouyama, T. Imamura, M. Nakamura, T. Satoh, Y. Futaana, Vertical propagation of planetary-scale waves in variable background winds in the upper cloud region of Venus. Icarus 248, 560-568 (2015). doi:10.1016/j.icarus.2014.07.011, 2015.

S. Lebonnois, F. Hourdin, V. Eymet, A. Crespin, R. Fournier, F. Forget, Super-rotation of Venus' atmosphere analyzed with a full general circulation model. J. Geophys. Res. 115, E06006 (2010). doi:10.1029/2009JE003458. 
2985

2986

2987

2988

2989

2990

2991

2992

2993

2994

2995

2996

2997

2998

2999

3000

3001

3002

3003

3004

3005

3006

3007

3008

3009

3010

3011

3012

3013

3014

3015

3016

3017

3018

3019

3020

3021

3022

3023

3024

3025

3026

3027

3028

3029

3030

S. Lebonnois, F. Hourdin, V. Eymet, A. Crespin, R. Fournier, F. Forget, Super-rotation of Venus' atmosphere analysed with a full General Circulation Model. J. of Geophys. Res. 115, E06006 (2010). doi:10.1029/2009JE003458

S. Lebonnois, C. Covey, A. Grossman, H. Parish, G. Schubert, R. Walterscheid, P. Lauritzen, C. Jablonowski, Angular momentum budget in General Circulation Models of superrotating atmospheres: A critical diagnostic. J. of Geophys. Res. 117, E12004 (2012). doi:10.1029/2012JE004223

S. Lebonnois, C. Lee, M. Yamamoto, J. Dawson, S. R. Lewis, J. Mendonca, P. L. Read, H. Parish, G. Schubert, L. Bengtsson, D. Grinspoon, S. Limaye, H. Schmidt, H. Svedhem, D. Titov, Models of Venus atmosphere, in Towards understanding the climate of Venus: Application of terrestrial models to our sister planet, ed. by L. Bengtsson, R.-M. Bonnet, D. Grinspoon, S. Koumoutsaris, S. Lebonnois, D. Titov. ISSI Scientific Report series 11 (Springer, Netherlands, 2013), pp. 129-156.

S. Lebonnois, N. Sugimoto and G. Gilli. Wave analysis in the atmosphere of Venus below 100km altitude, simulated by the LMD Venus GCM. Icarus 278, 38-51 (2016).

C. Lee, S. R. Lewis, P. L. Read, A numerical model of the atmosphere of Venus. Adv. Space Res. 36, 2142-2145 (2005). doi:10.1016/j.asr.2005.03.120

C. Lee, S. R. Lewis, P. L. Read, Super-rotation in a Venus general circulation model. J. of Geophys. Res. 112, E04S11 (2007). doi:10.1029/2006JE002874

C. Lee, S. R. Lewis, P. L. Read, A bulk cloud parameterization in a Venus General Circulation Model, Icarus, 206, 662-668 (2010)

C. Lee, M. I. Richardson, A General Circulation Model ensemble study of the atmospheric circulation of Venus. J. Geophys. Res. 115, E04002 (2010). doi:10.1029/2009JE003490

C. Lee, M. I. Richardson, A Discrete Ordinate, Multiple Scattering, Radiative Transfer Model of the Venus Atmosphere from 0.1 to 260 doi:10.1175/2011JAS3703.1

C. Lee, M. I. Richardson, Angular momentum conservation in a simplified Venus General Circulation Model. Icarus 221, 1173-1176 (2012). doi:10.1016/j.icarus.2012.10.007

E. Lellouch, T. Clancy, D. Crisp, A. Kliore, D. Titov, S. W. Bougher, Monitoring of Mesospheric Structure and Dynamics, in Venus II, eds. S. W. Bougher, D. M. Hunten, R. J. Phillips, (University of Arizona Press, Tucson, 1997) pp. 295-324.

E. Lellouch, , G. Paubert, R. Moreno, and a. Moullet, Monitoring Venus' Mesospheric Winds in Support of Venus Express: IRAM 30-M and APEX Observations, Plan. Space Sci. 56 (10): 1355-1367 (2008). doi:10.1016/j.pss.2008.06.010. 
C. B. Leovy, Rotation of the upper atmosphere of Venus, J. Atmos. Sci., 30, 1218-1220 (1973) doi:10.1175/1520-0469(1973)030

S.S. Leroy, A.P. Ingersoll, Convective generation of gravity waves in Venus's atmosphere: Gravity wave spectrum and momentum transport. J. Atmos. Sci. 52, 3717-3737 (1995).

S.S. Leroy, A.P. Ingersoll, Radio scintillations in Venus's atmosphere: Application of a theory of gravity wave generation. J. Atmos. Sci. 53, 1018-1028 (1996).

S. Limaye, V. Suomi, A Normalized View of Venus, J. Atmos. Sci., 34, 205-215 (1977)

S. S. Limaye, , and V. E. Suomi, Cloud Motions on Venus - Global Structure and Organization, J. Atmos. Scie. 38, 1220-1235 (1981)

S.S. Limaye, C.J. Grund, S.P. Burre, Zonal mean circulation at the cloud level on Venus: Spring and fall 1979 OCPP observations. Icarus 51, 416-439 (1982).

S. S. Limaye, Venus atmospheric circulation: observations and implications of the thermal structure, Adv. Space Res., 5(9), 51-62 (1985).

S.S. Limaye, Venus: Cloud level circulation during 1982 as determined from Pioneer cloud photopolarimeter images. II - solar longitude dependent circulation. Icarus 73, 212-226 (1988).

S. S. Limaye, Venus Atmospheric Circulation: Known and Unknown, J. Geophys. Res. 112, E04S09 (2007). doi:10.1029/2006JE002814.

S. S. Limaye, J. P. Kossin, C. Rozoff, G. Piccioni, D. V. Titov, W. J. Markiewicz, Vortex circulation on Venus: Dynamical similarities with terrestrial hurricanes. Geophys Res Lett 36, L04,204, (2009) doi:10.1029/2008GL036093

S S. Limaye, M. Rengel., Atmospheric Circulation and Dynamics of Venus, in Towards understanding the Climate of Venus, ed. by L. Bengtsson, R.-M. Bonnet, D. Grinspoon, S. Koumoutsaris, S. Lebonnois, D. Titov. ISSI Scientific Report series 11 (Springer, Netherlands, 2013), pp. 55-72.

V. M. Linkin, V. V. Kerzhanovich, A. N. Lipatov, K. M. Pichkadze, A. A. Shurupov, A. V. Tertebrashvili, A. P. Ingersoll, D. Crisp, A. W. Grossman, R. E. Young, A. Seiff, B. Ragent, J. E. Blamont, L. S. Elson, R. . Preston, VEGA Balloon Dynamics and Vertical Winds in the Venus Middle Cloud Region, Science 231, 1417 - 1419 (1986)

S. R. Lewis, C. Lee, P. L. Read, A Venus atmospheric general circulation model for Venus Express. European Planetary Science Congress, Sept. 18-22, Berlin, Germany (2006).

S. R. Lewis, J. Dawson, S. Lebonnois, M. Yamamoto, M., Modelling Efforts in Towards understanding the Climate of Venus, ed. by L. Bengtsson, R.-M. Bonnet, D. Grinspoon, S. 
3076

3077

3078

3079

3080

3081

3082

3083

3084

3085

3086

3087

3088

3089

3090

3091

3092

3093

3094

3095

3096

3097

3098

3099

3100

3101

3102

3103

3104

3105

3106

3107

3108

3109

3110

3111

3112

3113

3114

3115

3116

3117

3118

3119

3120

3121

Koumoutsaris, S. Lebonnois, D. Titov. ISSI Scientific Report series 11 (Springer, Netherlands, 2013), pp. 111-128.

R. S. Lindzen, Instability of plane parallel shear flow (towards a mechanistic picture of how it works). PAGEOPHYS, 16, 103-121 (1988)

M. A. López-Valverde, P. Drossart, R. Carlson, R. Mehlman, M. Roos-Serote, Non-LTE infrared observations at Venus: from NIMS/Galileo to VIRTIS/Venus Express. Planet. \& Sp. Sci. 55, 1757-1771 (2007). doi:10.1016/j.pss.2007.01.008

R. D. Lorenz, Surface winds on Venus: Probability distribution from in-situ measurements, Icarus, 264, 311-315 (2016)

D. Luz, D. L. Berry, G. Piccioni, P. Drossart, R. Politi, C. F. Wilson, S. Erard, F. Nuccilli. Venus's Southern Polar Vortex Reveals Precessing Circulation, Science 332, 577-580 (2011) doi:10.1126/science.1201629.

P. Machado, D. Luz, Th. Widemann, E. Lellouch, O. Witasse, Mapping zonal winds at Venus's cloud tops from ground-based Doppler velocimetry, Icarus 221, 248-261 (2013)

P. Machado, Th. Widemann, D. Luz, J. Peralta, J.. Wind circulation regimes at Venus' cloud tops: Ground-based Doppler velocimetry using CFHT/ESPaDOnS and comparison with simultaneous cloud tracking measurements using VEx/VIRTIS in February, 2011. Icarus 243, 249-263 (2014)

P. Machado, T. Widemann, J. Peralta, R. Gonçalves, J.-F. Donati, D. Luz. Venus cloud-tracked and doppler velocimetry winds from CFHT/ESPaDOnS and Venus Express/VIRTIS in April 2014. Icarus, 285, 8-26 (2017)

W. J. Markiewicz, D. V. Titov, S. S. Limaye, H. U. Keller, N. Ignatiev, R. Jaumann, N. Thomas, H. Michalik, R. Moissl \& P. Russo, Morphology and dynamics of the upper cloud layer of Venus, Nature 450, 633-636. 2007.

M. Y. Marov, Results of Venus Missions, Annual Review of Astronomy and Astrophysics 16, 141-169 (1978). doi:10.1146/annurev.aa.16.090178.001041.

H. G. Mayr, I. Harris, Quasi-axisymmetric circulation and super-rotation in planetary atmospheres. Astron. \& Astrophys. 121, 124-136 (1983).

J. M. Mendonca, P. L. Read, C. F. Wilson and S. R. Lewis, Zonal winds at high latitudes on Venus: An improved application of cyclostrophic balance to Venus Express observations. Icarus, 217, 629-639 (2012) doi:10.1016/j.icarus.2011.07.010

J. M. Mendonca, P. L. Read, C. F. Wilson, C. Lee, A new fast and flexible radiative transfer method for Venus general circulation models. Planet. \& Space Sci. 105, 80-93 (2015). doi:10.1016/j.pss.2014.11.008 
J. M. Mendonca and P. L. Read, Exploring the Venus global super-rotation using a

\section{V. Michelangeli, R. W. Zurek, L. S. Elson, Barotropic instability of midlatitude zonal jets on} Mars, Earth and Venus. J. Atmos. Sci., 44, 2031-2041 (1987)

A. Migliorini , D. Grassi, L. Montabone, S. Lebonnois, P. Drossart, G. Piccioni, Investigation of air temperature on the nightside of Venus derived from VIRTIS-H on board Venus-Express. Icarus 217, 640-647 (2012). doi:10.1016/j.icarus.2011.07.013

J.L. Mitchell, G.K. Vallis, The transition to super-rotation in terrestrial atmospheres. J. Geophys. Res. 115, E12008 (2010). doi:10.1029/2010JE003587

R. Moissl, I. Khatuntsev, S. S. Limaye, D. V. Titov, W. J. Markiewicz, N. I. Ignatiev, T Roatsch, et al., Venus Cloud Top Winds from Tracking UV Features in Venus Monitoring Camera Images, J. Geophys. Res.-Planets 114, E00B31 (2009). doi:10.1029/2008JE003117.

V. I. Moroz, The Atmosphere of Venus, Space Sci. Rev. 29, 3-127 (1981).

V. I. Moroz, L. V. Zasova, VIRA-2: A review of inputs for updating the Venus International Reference Atmosphere, Adv. Space Res., 19, 1191 - 1201 (1997).

M. Newman, G. Schubert, A.J. Kliore, I.R. Patel, Zonal winds in the middle atmosphere of Venus from Pioneer Venus radio occultation data. J. Atmos. Sci. 41, 1901-1913 (1984).

M. Newman, C. Leovy, Maintenance of strong rotational winds in Venus' middle atmosphere by thermal tides. Science 257, 647-650 (1992).

G.S. Orton, J. Caldwell, A.J. Friedson, T.Z. Martin, Middle infrared thermal maps of Venus at the time of the Galileo encounter, Science 253, 1536-1538 (1991)

H. F. Parish, G. Schubert, C. Covey, R. L. Walterscheid, A. Grossman, S. Lebonnois, Decadal variations in a Venus General Circulation Model. Icarus 212, 42-65 (2011). doi:10.1016/ j.icarus.2010.11.015

J.B. Pechmann, A.P. Ingersoll, Thermal tides in the atmosphere of Venus: Comparison of model results with observations. J. Atmos. Sci. 41, 3290-3313 (1984).

J. Peralta, R. Hueso, A. Sánchez-Lavega, Cloud brightness distribution and turbulence in Venus using Galileo Violet images, Icarus 188, 305-314 (2007). doi:10.1016/j.icarus.2007.03.028.

J. Peralta, R. Hueso, A. Sánchez-Lavega, A reanalysis of Venus winds at two cloud levels from Galileo SSI images, Icarus 190, 469-477 (2007). doi:10.1016/j.icarus.2007.03.028. 
J. Peralta, R. Hueso, A. Sanchez-Lavega, G. Piccioni, O. Lanciano, and P. Drossart, Characterization of mesoscale gravity waves in the upper and lower clouds of Venus from VEXVIRTIS images, J. Geophys. Res. 113, E00B18, doi:10.1029/2008JE003185 (2008). (2012). doi:10.1016/j.icarus.2012.06.015.

J. Peralta, T. Imamura, P.L. Read, D. Luz, A. Piccialli, M.A. López-Valverde, Analytical solution for waves in planets with atmospheric super-rotation. I. acoustic and inertia-gravity waves. Astrophys. J. Suppl. Ser. 213, 17 (2014a). doi:10.1088/0067-0049/213/1/17

J. Peralta, T. Imamura, P.L. Read, D. Luz, A. Piccialli, M.A. López-Valverde, Analytical solution for waves in planets with atmospheric super-rotation. II. Lamb, surface, and centrifugal waves. Astrophys. J. Suppl. Ser. 213, 18 (2014b). doi:10.1088/0067-0049/213/1/18

J. Peralta, A. Sanchez-Lavega, M. A. Lopez-Valverde, D. Luz, and P. Machado, Venus's major cloud feature as an equatorially-trapped wave distorted by the wind, Geophys. Res. Lett. 42, 705711 (2015).

J. Peralta, M. A. Lopez-Valverde, G. Gilli, and A. Piccialli, Dayside temperatures in the Venus upper atmosphere from Venus Express/VIRTIS nadir measurements at 4.3 microns. Astron. \& Astrophys. 585, A53 (2016). doi:10.1051/0004-6361/201527191.

J. Peralta, Y. Joo Lee, K. McGouldrick, H. Sagawa, A. Sanchez-Lavega, T. Imamura, T. Widemann, M. Nakamura, "Overview of useful spectral regions for Venus: an update to encourage observations complementary to the Akatsuki mission”, Icarus, 288, 235-239 (2017)

A. Petculescu, R.M. Lueptow, Atmospheric acoustics of Titan, Mars, Venus, and Earth, Icarus 186, 413-419 (2007).

A. Piccialli, AD. V. Titov, D. Grassi, I. Khatuntsev, P. Drossart, G. Piccioni,5 and A. Migliorini, Cyclostrophic winds from the Visible and Infrared Thermal Imaging Spectrometer temperature sounding: A preliminary analysis, J. Geophys. Res., 113, E00B11, (2008) doi:10.1029/2008JE003127.

A. Piccialli, S. Tellmann, D.V. Titov, S.S. Limaye, I.V. Khatuntsev, M. Pätzold and B. Häusler, Dynamical properties of the Venus mesosphere from the radio-occultation experiment VeRa onboard Venus Express, Icarus, 217, 669-681 (2012) doi:10.1016/j.icarus.2011.07.016

A. Piccialli A., D. V. Titov, A. Sanchez-Lavega, J. Peralta, O. Shalygina, W. J. Markiewicz, H. Svedhem, High latitude gravity waves at the Venus cloud tops as observed by the Venus Monitoring Camera on board Venus Express, Icarus 227, 94-111 (2014). 
3212

3213

3214

3215

3216

3217

3218

3219

3220

3221

3222

3223

3224

3225

3226

3227

3228

3229

3230

3231

3232

3233

3234

3235

3236

3237

3238

3239

3240

3241

3242

3243

3244

3245

3246

3247

3248

3249

3250

3251

3252

3253

3254

3255

3256

3257

G. Piccioni, P. Drossart, A. Sanchez-Lavega, R. Hueso, et al., South polar features on Venus similar to those near the north pole, Nature 450, 637-640 (2007).

R. A. Plumb, Angular momentum advection by axisymmetric motions. Quart. J. R. Meteorol. Soc., 103, 479-485 (1977).

J.B. Pollack, O.B. Toon, R.C. Whitten, R. Boese, B. Ragent, M. Tomasko, L. Esposito, L. Travis, D. Wiedman, Distribution and source of the UV absorption in Venus' atmosphere. J. Geophys. Res. 85, 8141-8150 (1980).

C. H. B. Prieslty, Turbulent Transfer in the lower atmosphere (Chicago University Press, Chicago, 1959)

B. Ribstein, V. Zeitlin, A.-S. Tissier, Barotropic, baroclinic and inertial instabilities of the easterly Gaussian jet on the equatorial Đplane in rotating shallow water, Phys. Fluids, 26, 056605 (2014) doi: 10.1063/1.4875030

V. Ramanathan, R.D. Cess, An analysis of the strong zonal circulation within the stratosphere of Venus. Icarus 25, 89-103 (1975).

P. L. Read, Super-rotation and diffusion of axial angular momentum: II. a review of quasiaxisymmetric models of planetary atmospheres. Quart J R Meteorol. Soc 112, 253-272 (1986)

P. L. Read, The Dynamics and Circulation of Venus Atmosphere, in Towards understanding the Climate of Venus, L. Bentgsson et al. eds., Springer, New York, 2013), pp. 73-110.

C. Roldan , M. A. Lopez-Valverde, M. Lopez-Puertas, D. P. Edwards., Non-LTE Infrared Emissions of $\mathrm{CO}_{2}$ in the Atmosphere of Venus. Icarus 147, 11-25 (2000). doi:10.1006/icar.2000.6432

M. Roos-Serote, P. Drossart, Th. Encrenaz, E. Lellouch, R. W. Carlson, K. H. Baines, F. W. Taylor, S. B. Calcutt, Thermal structure and dynamics of the atmosphere of Venus between 70 and $90 \mathrm{~km}$ from the Galileo-NIMS spectra, Icarus, 114, 300-309 (1995).

W. B. Rossow, G. P. Williams, Large scale motions in the Venus stratosphere. J. Atmos. Sci. 36, 377-389 (1979).

W. B. Rossow, A. Del Genio, S. S. Limaye, L. D. Travis, Cloud morphology and motions from Pioneer Venus images, J. Geophys. Res., 85, 8107 - 8128 (1980)

W. B. Rossow, S. B. Fels, P. H. Stone, Comments on 'A three-dimensional model of dynamical processes in the Venus atmosphere'. J. Atmos. Sci., 37, 250-252 (1980).

W. B. Rossow, A general circulation model of a Venus-like atmosphere. J. Atmos. Sci., 40, $273-$ 302 (1983). doi:10.1175/1520-0469 
W. B. Rossow, A. D. del Genio, T. Eichler, Cloud-tracked winds from Pioneer Venus OCPP images, J. Atmos. Sci. 47, 2053-2084 (1990).

A. Sánchez-Lavega, R. Hueso, G. Piccioni, P. Drossart, J. Peralta, S. Pérez-Hoyos, C. Wilson, F. Taylor, K. Baines, D. Luz, S. Erard, S. Lebonnois, Variable winds on Venus mapped in three dimensions, Geophys. Res. Lett. 35, L13204, doi:10.1029/2008GL033817 (2008).

A. Sánchez-Lavega, An Introduction to Planetary Atmospheres, Taylor-Francis, CRC Press Boca Raton, Florida (2011)

A. Sánchez-Lavega, J. Peralta, J. M. Gómez-relad, R. Hueso, S. Pérez-Hoyos, I. Mendikoa, J. F. Rojas, T. Horinouchi, Y. J. Lee, S. Watanabe, Venus cloud morphology and motions from ground-based images at the time of the Akatsuki orbit insertion, Astrophys. J. Lett. , 833:L7 (7pp) (2016).

R. Z. Sagdeev, et al., Overview of VEGA Venus balloon in situ meteorological measurements. Science 231, 1411-1414 (1986).

S. Sakai, Rossby-Kelvin instability: A new type of ageostrophic instability caused by a resonance between Rossby waves and gravity waves, J. Fluid Mech. 202, 149-176 (1989).

T. M. Sato, H. Sawada, T. Kouyama, K. Mitsuyama, T. Satoh, S. Ohtsuki, M. Ueno, Y. Kasaba, M. Nakamura, T. Imamura, Cloud top structure of Venus revealed by Subaru/COMICS midinfrared images, Icarus, 243, 386-399 (2014).

P.J. Schinder, P.J. Gierasch, S.S. Leroy, M.D. Smith, Waves, advection, and cloud patterns on Venus. J. Atmos. Sci. 47, 2037-2052 (1990).

E. K. Schneider, Axially symmetric steady state models of the basic state for instability and climate studies. part II. nonlinear calculations. J Atmos Sci 34:280-296 (1977).

J. T. Schofield, D. J. Diner, Rotation of Venus's Polar Dipole, Nature 305, 116-119 (1983). doi:10.1038/305116a0

J.T. Schofield, F.W. Taylor, Measurements of the mean, solar-fixed temperature and cloud structure of the middle atmosphere of Venus. Quart. J. R. Met. Soc. 109, 57-80 (1983).

G. Schubert, C. Covey, A. Del Genio, L. S. Elson, G. Keating, A. Seiff, R. E. Young, J. Apt, C. C. Counselman, A. J. Kliore, S.S. Limaye, H. E. Revercomb, L. A. Sromovsky, V . E. Suomi, F. Taylor, R. Woo, and U. Von Zahn, Structure and Circulation of the Venus Atmosphere, J. Greophys. Res., 85,(A13), 8007-8025 (1980).

G. Schubert, General Circulation and the Dynamical State of the Venus Atmosphere, in Venus I, edts. D. M. Hunten, L. Colin, T. M. Donahue, V. I. Moroz, (University of Arizona Press, Tucson, 1983) pp. 681-765. 
G. Schubert, R.L. Walterscheid, Propagation of small-scale acoustic-gravity waves in the Venus atmosphere. J. Atmos. Sci. 41, 1202-1213 (1984).

A. Seiff, D.B. Kirk, R.E. Young, R.C. Blanchard, J.T. Findlay, G.M. Kelly, S.C. Sommer, Measurements of thermal structure and thermal contrasts in the atmosphere of Venus and related dynamical observations: Results From the four Pioneer Venus Probes. J. Geophys. Res. 85, 7903-7933, (1980).

3309

3310

3311

3312

3313

3314

3315

3316

3317

3318

3319

3320

3321

3322

3323

3324

3325

3326

3327

3328

3329

3330

3331

3332

3333

3334

3335

3336

3337

3338

3339

3340

3341

3342

3343

3344

3345

3346

3347

3348

3349

A. Seiff, Dynamical implications of the observed thermal contrasts in Venus's upper atmosphere. Icarus 51, 574-592 (1982).

M. D. Smith, P.J. Gierasch, P.J. Schinder, Global-scale waves in the Venus atmosphere. J. Atmos. Sci. 50, 4080-4096 (1993).

M. D. Smith, P.J. Gierasch, P.J. Schinder, Science, A global traveling wave on venus. Science 256, 652-655 (1992).

M. D. Smith, P.J. Gierasch, P.J. Schinder, Global-scale waves in the Venus atmosphere. J. Atmos. Sci., 50, 4080-4096 (1993).

M. Sornig et al., Venus upper atmosphere winds from ground-based heterodyne spectroscopy of $\mathrm{CO}_{2}$ at $10 \mu \mathrm{m}$ wavelength, Plan. Space Sci. 56, 1399-1406 (2012).

N. Sugimoto, M. Takagi, Y. Matsuda, Baroclinic instability in the Venus atmosphere simulated by GCM. J. Geophys. Res. (Planets) 119, 1950-1968 (2014a). doi:10.1002/2014JE004624

N. Sugimoto, M. Takagi, Y. Matsuda, Waves in a Venus general circulation model. Geophys. Res. Lett. 41, 7461-7467 (2014b). doi:10.1002/2014GL061807

V. E. Suomi, S. S. Limaye, Venus - Further Evidence of Vortex Circulation, Science 201, 10091111 (1978).

M. Takagi, Y. Matsuda, Sensitivity of thermal tides in the Venus atmosphere to basic zonal flow and Newtonian cooling. Geophys. Res. Lett. 32, L02203 (2005). doi:10.1029/2004GL022060.

M. Takagi, Y. Matsuda, A further study on the stability of a baroclinic flow in cyclostrophic balance. Geophys. Res. Lett. 32, L19804, (2005b). doi:10.1029/2005GL023700.

M. Takagi, Y. Matsuda, Dynamical effect of thermal tides in the lower Venus atmosphere. Geophys. Res. Lett. 33, L13102 (2006). doi:10.1029/2006GL026168.

M. Takagi, Y. Matsuda, A study on the stability of a baroclinic flow in cyclostrophic balance on the sphere. Geophys. Res. Lett. 33, L14807, (2006b). doi:10.1029/2006GL026200.

M. Takagi, Y. Matsuda, Effects of thermal tides on the Venus atmospheric super-rotation. J. Geophys. Res. 112, D09112 (2007). doi:10.1029/2006JD007901. 
F. W. Taylor, D. J. Diner, L. S. Elson, M. S. Hanner, D. J. McCleese, J. V. Martonchik, P. E. Orbiter, Science 203, 779-781 (1979). doi:10.1126/science.203.4382.779.

F. W. Taylor, R. Beer, M. T. Chahine, D. J. Diner, L. S. Elson, R. D. Haskins, D. J. McCleese, J. V. Martonchik, P. E. Reichley, S. P. Bradley, J. Delderfield, J. T. Schofield, C. B. Farmer, L. Froidevaux, J. Leung, M. T. Coffey, J. C. Gille, Structure and meteorology of the middle atmosphere of Venus: Infrared remote sounding from the Pioneer Orbiter. J. Geophys. Res. 85, 7963-8006 (1980).

F. W. Taylor, The Scientific Exploration of Venus, Cambridge University Press, Cambridge (2014).

S. Tellmann, M. Pätzold, B. Häusler, M.K. Bird, G.L. Tyler, Structure of the Venus neutral atmosphere as observed by the radio science experiment VeRa on Venus Express. J. Geophys. Res. 114, E00B36 (2009).

S. Tellmann, B. Häusler, D.P. Hinson, G.L. Tyler, T.P. Andert, M.K. Bird, T. Imamura, M. Pätzold, S. Remus, Small-scale temperature fluctuations seen by the VeRa radio science experiment on Venus Express. Icarus 221, 471-480 (2012).

D. V. Titov et al., Morphology of the cloud tops as observed by the Venus Express Monitoring Camera. Icarus 217, 682-701 (2012).

A. Toigo, P. J. Gierasch, M. D. Smith, High resolution cloud feature tracking on Venus by Galileo, Icarus 109, 318-336 (1994).

M. G. Tomasko, L.R. Doose, P.H. Smith, A.P. Odell, Measurements of the flux of sunlight in the atmosphere of Venus. J. Geophys. Res. 85, 8167-8186 (1980).

L. D. Travis, Nature of the atmospheric dynamics on Venus from power spectrum analysis of Mariner 10 images, J. Atmos. Sci., 35, 1584-1595 (1978).

G. K. Vallis, Atmospheric and Oceanic Fluid Dynamics (Cambridge University Press, UK), (2006)

M. Yamamoto, H. Tanaka, Formation and Maintenance of the 4-Day Circulation in the Venus Middle Atmosphere. J. Atmos. Sci. 54, 1472-1489 (1997).

M. Yamamoto, M. Takahashi, The Fully Developed Super-rotation Simulated by a General Circulation Model of a Venus-like Atmosphere. J. Atmos. Sci., 60, 561-574 (2003a).

M. Yamamoto, M. Takahashi, Super-rotation and equatorial waves in a T21 Venus-like AGCM. Geophys. Res. Lett. 30, 1449 (2003b). doi:10.1029/2003GL016924 
M. Yamamoto, M. Takahashi, Dynamics of Venus' super-rotation: The eddy momentum transport processes newly found in a GCM. Geophys. Res. Lett. 31, L09701 (2004). doi:10.1029/2004GL019518

M. Yamamoto, M. Takahashi, Super-rotation Maintained by Meridional Circulation and Waves in a Venus-Like AGCM. J. of Atm. Sci. 63, 3296-3314 (2006).

M. Yamamoto, M. Takahashi, Dynamical effects of solar heating below the cloud layer in a Venus-like atmosphere. J. of Geophys. Res. 114, E12004 (2009). doi:10.1029/2009JE003381

M. Yamamoto, M. Takahashi, Venusian middle-atmospheric dynamics in the presence of a strong planetary-scale 5.5-day wave. Icarus 217, 702-713 (2012).

doi:10.1016/j.icarus.2011.06.017

M. Yamamoto and M. Takahashi, Dynamics of polar vortices at cloud top and base on Venus inferred from a general circulation model: Case of a strong diurnal thermal tide. Planetary and Space Science, 113, 109-119 (2015)

R. E. Young, J. B. Pollack, A three-dimensional model of dynamical processes in the Venus atmosphere. J. of Atm. Sci. 34, 1315-1351 (1977).

R. E. Young, H. Houben, L. Pfister, Baroclinic instability in the Venus atmosphere. J. Atmos. Sci., 41, 2310-2333 (1984)

R. E. Young, R.L. Walterscheid, G. Schubert, A. Seiff, V.M. Linkin, A.N. Lipatov, Characteristics of gravity waves generated by surface topography on Venus: Comparison with the VEGA blloon results. J. Atmos. Sci. 44, 2628-2639 (1987).

R. E. Young, R.L. Walterscheid, G. Schubert, L. Pfister, H. Houben, D.L. Bindschadler, Characteristics of finite amplitude stationary gravity waves in the atmosphere of Venus. J. Atmos. Sci. 51, 1857-1875 (1994).

T. Widemann, et al., New wind measurements in Venus lower mesosphere from visible spectroscopy, Planet. Space Sci. 55, 1741-1756 (2007).

T. Widemann et al., Venus Doppler winds at cloud tops observed with ESPaDOnS at CFHT, Planet. Space Sci. 56, 1320-1334 (2008).

A. M. Zalucha, A. S. Brecht, S. Rafkin, S. W. Bougher, M. J. Alexander, Incorporation of a gravity wave momentum deposition parameterization into the Venus Thermosphere General Circulation Model (VTGCM). J. of Geophys. Res. (Planets) 118, 147-160 (2013). doi:10.1029/2012JE004168

L. Zasova, I.V. Khatountsev, N.I. Ignatiev, V.I. Moroz, Local time variations of the middle atmosphere of Venus: Solar-related structures. Adv. Space Res. 29, 243-248 (2002). doi:10.1016/S0273-1177(01)00574-9. 
3443 L.V. Zasova, N. Ignatiev, I. Khatuntsev, V. Linkin, Structure of the Venus atmosphere. Planet. 3444 Space Sci. 55, 1712-1728 (2007). doi:10.1016/j.pss.2007.01.011.

3445

3446 S. Zhang, S.W. Bougher, M.J. Alexander, The impact of gravity waves on the Venus 3447 3448

3449

3450

3451 thermosphere and $\mathrm{O}_{2}$ IR nightglow. J. Geophys. Res. 101, 23195-23205 (1996). 